\title{
Radiation-Hardened Circuitry Using Mask-Programmable Analog Arrays: Final Report
}

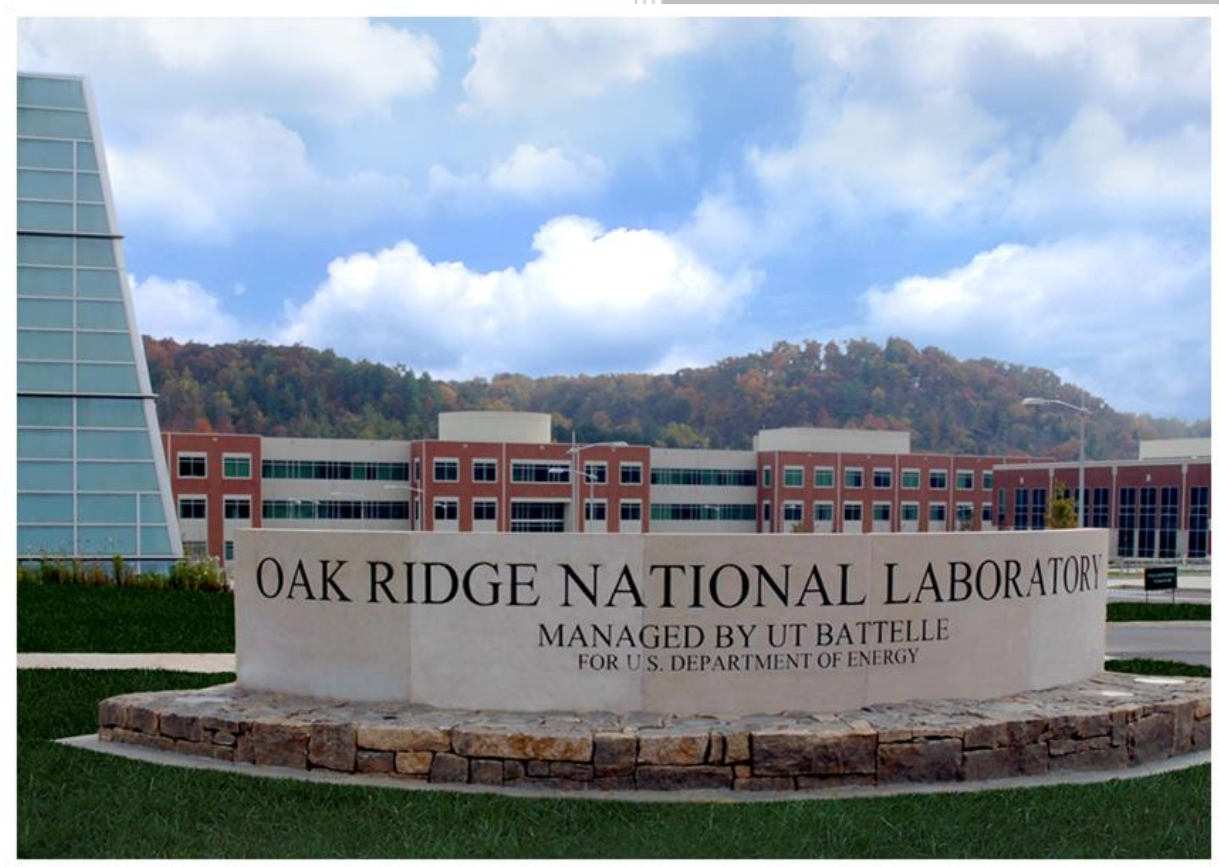

Approved for public release.

C. L. Britton

J. Shelton

M. N. Ericson

M. Bobrek

B. Blalock

Distribution is unlimited.

December 2015 


\section{DOCUMENT AVAILABILITY}

Reports produced after January 1, 1996, are generally available free via US Department of Energy (DOE) SciTech Connect.

Website http://www.osti.gov/scitech/

Reports produced before January 1, 1996, may be purchased by members of the public from the following source:

National Technical Information Service

5285 Port Royal Road

Springfield, VA 22161

Telephone 703-605-6000 (1-800-553-6847)

TDD 703-487-4639

Fax 703-605-6900

E-mailinfo@ntis.gov

Website http://www.ntis.gov/help/ordermethods.aspx

Reports are available to DOE employees, DOE contractors, Energy Technology Data Exchange representatives, and International Nuclear Information System representatives from the following source:

Office of Scientific and Technical Information

PO Box 62

Oak Ridge, TN 37831

Telephone 865-576-8401

Fax 865-576-5728

E-mail reports@osti.gov

Website http://www.osti.gov/contact.html

This report was prepared as an account of work sponsored by an agency of the United States Government. Neither the United States Government nor any agency thereof, nor any of their employees, makes any warranty, express or implied, or assumes any legal liability or responsibility for the accuracy, completeness, or usefulness of any information, apparatus, product, or process disclosed, or represents that its use would not infringe privately owned rights. Reference herein to any specific commercial product, process, or service by trade name, trademark, manufacturer, or otherwise, does not necessarily constitute or imply its endorsement, recommendation, or favoring by the United States Government or any agency thereof. The views and opinions of authors expressed herein do not necessarily state or reflect those of the United States Government or any agency thereof. 
Nuclear Security and Isotope Technology Division

\title{
RADIATION-HARDENED CIRCUITRY USING MASK-PROGRAMMABLE ANALOG ARRAYS: FINAL REPORT
}

\author{
C. L. Britton \\ J. Shelton \\ M. N. Ericson \\ M. Bobrek \\ B. Blalock
}

Date Published: December 2015

\author{
Prepared by \\ OAK RIDGE NATIONAL LABORATORY \\ Oak Ridge, TN 37831-6283 \\ managed by \\ UT-BATTELLE, LLC \\ for the \\ US DEPARTMENT OF ENERGY \\ under contract DE-AC05-00OR22725
}





\section{CONTENTS}

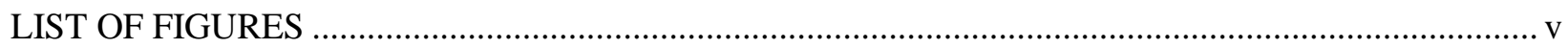

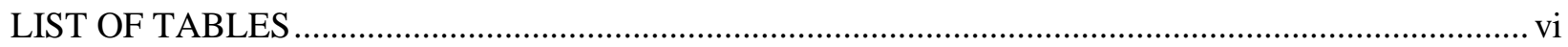

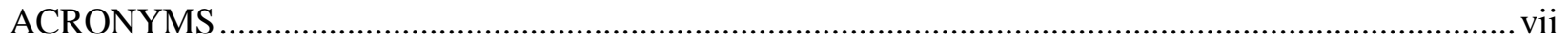

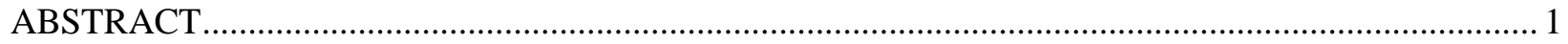

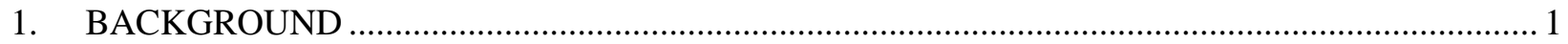

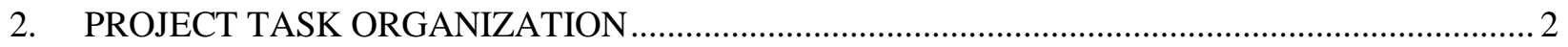

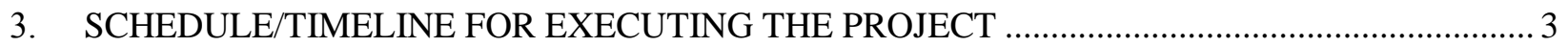

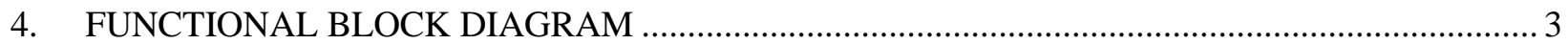

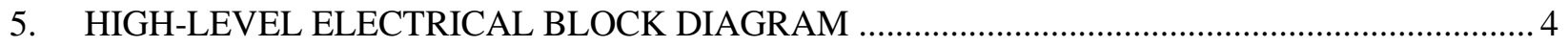

6. SENSORS AND SUPPORT COMPONENT EVALUATION ….............................................. 5

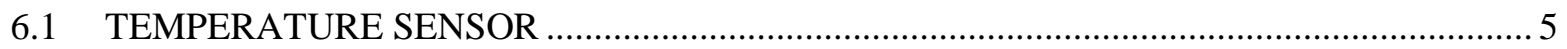

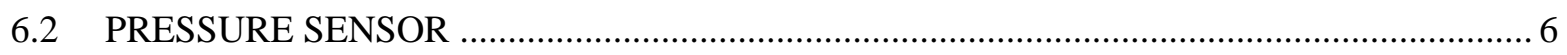

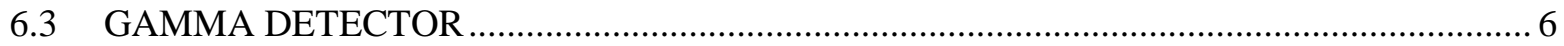

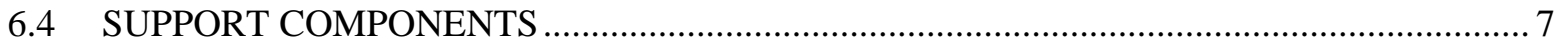

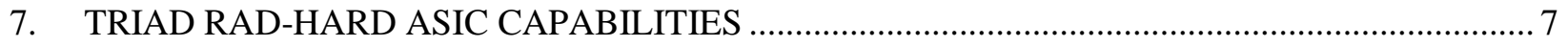

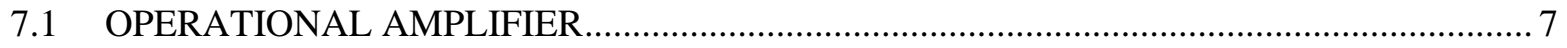

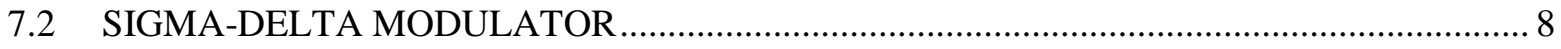

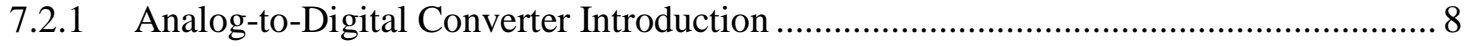

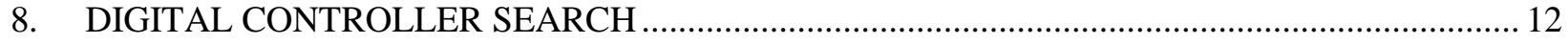

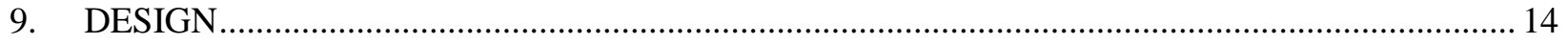

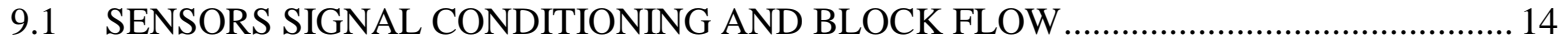

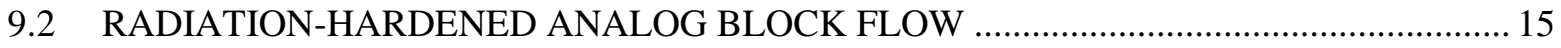

9.3 NON-RADIATION-HARDENED CONTROL BLOCK FLOW ....................................... 16

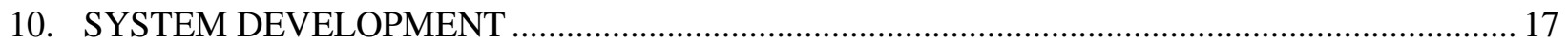

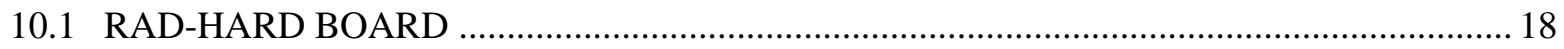

10.1.1 Voltage Regulator and Frequency Synthesizer .................................................... 19

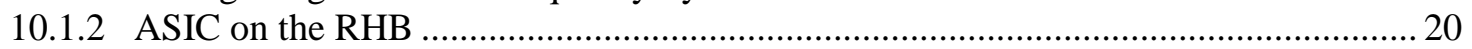

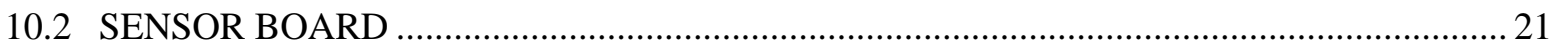

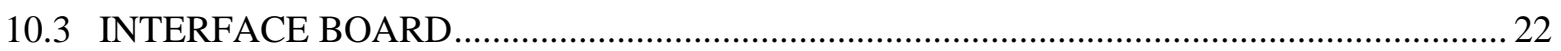

10.4 NEXYS 3 BOARD FIRMWARE/COMPUTER INTERFACE ........................................... 23

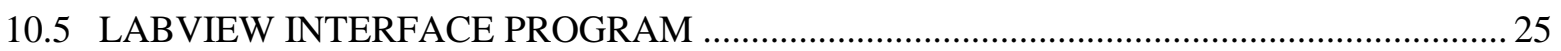

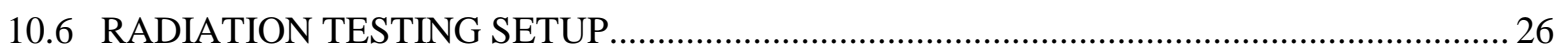

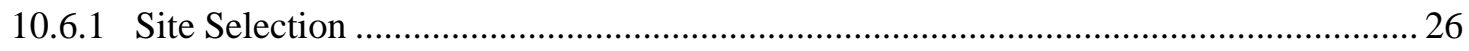

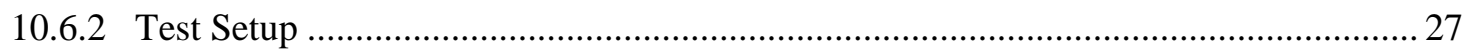

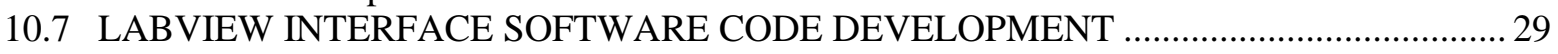

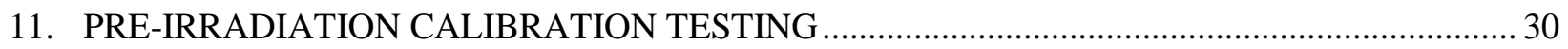

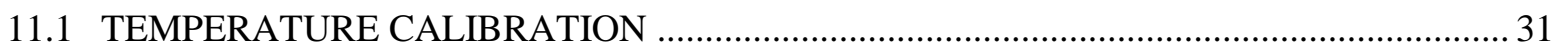

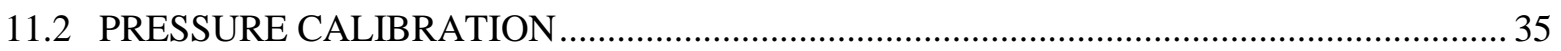

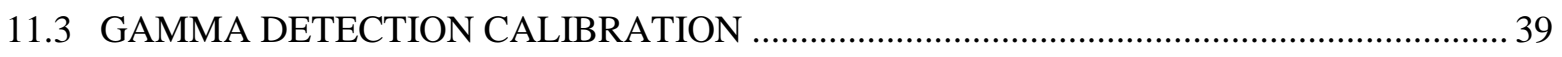

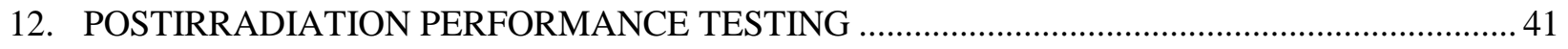

12.1 CURRENT CONSUMPTION RADIATION PERFORMANCE ........................................ 42

12.2 TEMPERATURE DATA RADIATION PERFORMANCE ............................................ 44

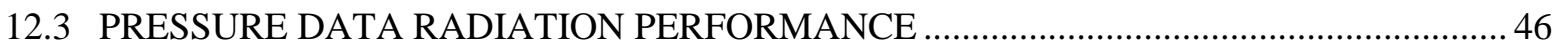

12.4 GAMMA EVENT DATA RADIATION PERFORMANCE ….................................... 48

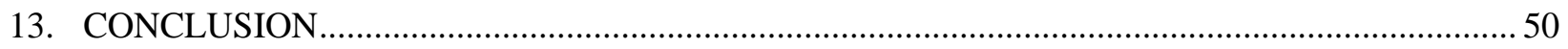

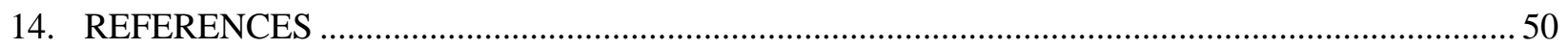





\section{LIST OF FIGURES}

Fig. 1. System functional description diagram. ......................................................................... 4

Fig. 2. Data acquisition system based on commercial and near-commercial rad-hard circuits................... 5

Fig. 3. Noise shaping to move much of the quantization noise out of the signal band.............................. 9

Fig. 4. Effect of quantization noise shaping and oversampling on the in-band noise in a sigmadelta ADC. The left plot shows a higher oversampling ratio than shown on the right. The signal bandwidth is marked with dashed lines...................................................................... 9

Fig. 5. Theoretical ADC resolution as a function of oversampling ratio and noise-shaping order............ 10

Fig. 6. Digital filtering of the signal bandwidth following oversampling and noise shaping................... 11

Fig. 7. Sigma-delta ADC block diagram............................................................................................ 11

Fig. 8. Second-order sigma-delta modulator implementation using switched capacitor circuits................ 11

Fig. 9. Second-order sinc filter topology (with decimation) ............................................................... 12

Fig. 10. Development kits purchased for the digital controller development: Digilent Nexys-3 Spartan-6 XC6SLX16-CS324 (left) and Xilinx CoolRunner-II Evaluation Board with

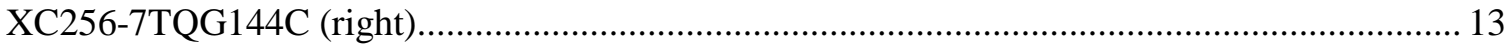

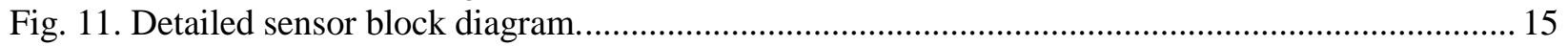

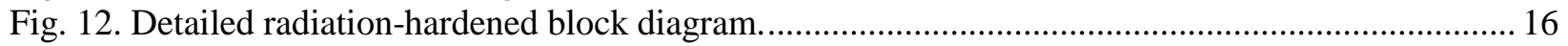

Fig. 13. Detailed Soft (non-rad hard) Block Diagram. .................................................................... 17

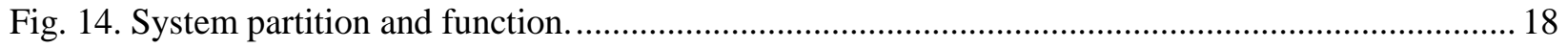

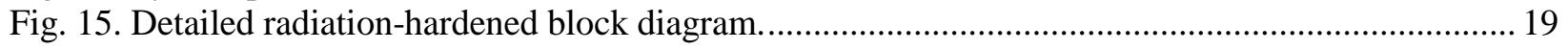

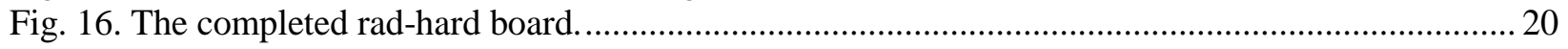

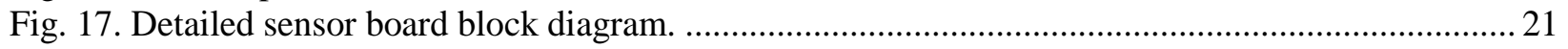

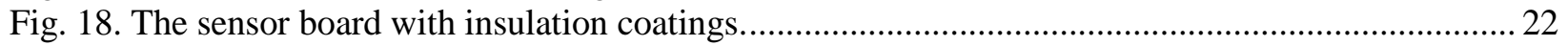

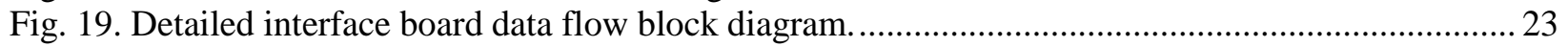

Fig. 20. The interface board (right) connected to the Nexys 3 board. ................................................. 23

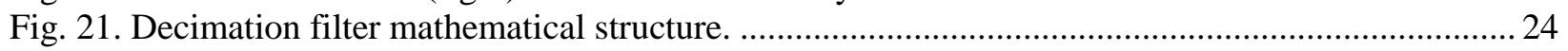

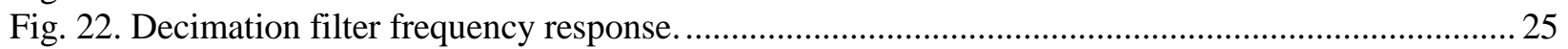

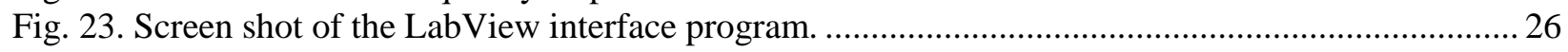

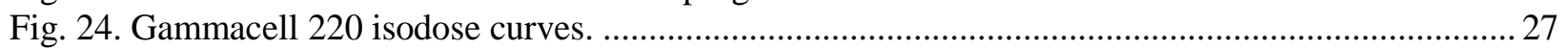

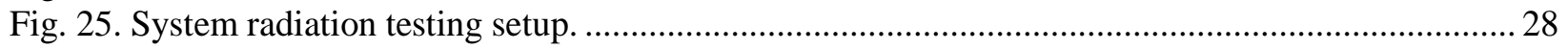

Fig. 26. LabVIEW interface software used for calibration and radiation testing. .................................... 30

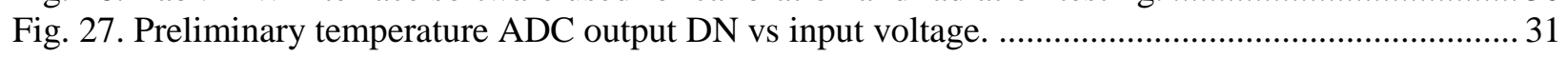

Fig. 28. System 1 temperature output DN vs ambient temperature ................................................... 32

Fig. 29. System 2 temperature output DN vs ambient temperature. .............................................. 33

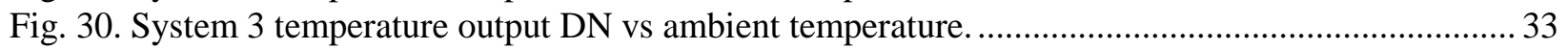

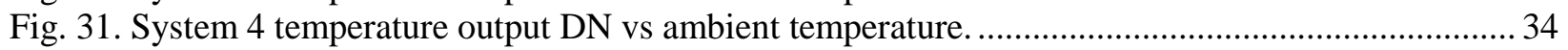

Fig. 32. System 5 temperature output DN vs ambient temperature ................................................... 34

Fig. 33. Preliminary pressure ADC output DN vs input voltage. ...................................................... 36

Fig. 34. System 1 pressure output DN vs ambient pressure........................................................ 37

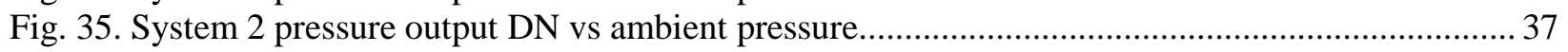

Fig. 36. System 3 pressure output DN vs ambient pressure............................................................. 38

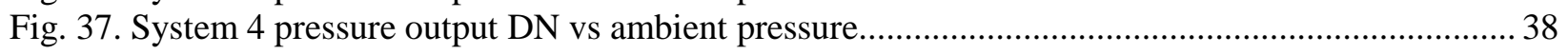

Fig. 38. System 5 pressure output DN vs ambient pressure.......................................................... 39

Fig. 39. Gamma event and comparator output pulse response. ............................................................ 40

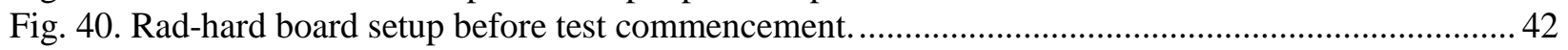

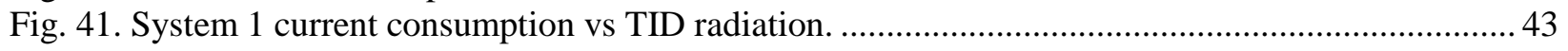

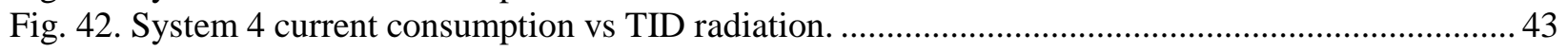

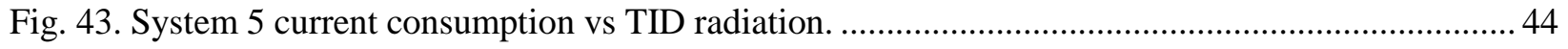

Fig. 44. System 1 temperature reading vs TID radiation. ....................................................................... 45 
Fig. 45. System 4 temperature reading vs TID radiation. 45

Fig. 46. System 5 temperature reading vs TID radiation. .46

Fig. 47. System 1 pressure reading vs TID radiation.

Fig. 48. System 4 pressure reading vs TID radiation.

Fig. 49. System 5 pressure reading vs TID radiation.

Fig. 50. System 1 gamma count rate vs TID radiation

Fig. 51. System 4 gamma count rate vs TID radiation

\section{LIST OF TABLES}

Table 1. Project Timeline.

Table 2. Theoretical Sigma Delta ADC resolution as a function of modulator order (L) and

oversampling ratio $(\mathrm{M})$

Table 3. Comparison of commercially available rad-hard or rad-tolerant FPGAs

Table 4. Summary of system temperature calibrations fit values and coefficients

Table 5. Summary of system pressure calibrations fit values and coefficients 


\section{ACRONYMS}

$\begin{array}{ll}\text { ADC } & \text { analog-to-digital converter } \\ \text { ASIC } & \text { application-specific integrated circuit } \\ \text { CIC } & \text { Cascade, Integrate and Comb } \\ \text { CPLD } & \text { complex programmable logic devices } \\ \text { DN } & \text { digital number } \\ \text { FMI } & \text { Frequency Management International } \\ \text { FPGA } & \text { a field-programmable gate array } \\ \text { G-M } & \text { Geiger-Müeller } \\ \text { IB } & \text { interface board } \\ \text { IC } & \text { integrated circuits } \\ \text { NiMH } & \text { nickel metal hydride } \\ \text { ORNL } & \text { Oak Ridge National Laboratory } \\ \text { PCB } & \text { printed circuit board } \\ \text { RHB } & \text { rad-hard board } \\ \text { RHBD } & \text { radiation-hardened-by-design } \\ \text { TID } & \text { total integrated dose } \\ \text { UART } & \text { universal asynchronous receive transmitter } \\ \text { USB } & \text { universal serial bus } \\ \text { VCA } & \text { via-configured array } \\ & \end{array}$





\begin{abstract}
As the recent accident at Fukushima Daiichi so vividly demonstrated, telerobotic technologies capable of withstanding high-radiation environments need to be readily available to enable operations, repair, and recovery under severe accident scenarios where human entry is extremely dangerous or not possible. Telerobotic technologies that enable remote operation in high-dose-rate environments have undergone revolutionary improvement over the past few decades. However, much of this technology cannot be employed in nuclear power environments due the radiation sensitivity of the electronics and the organic insulator materials currently in use.
\end{abstract}

This is the final report of the activities involving the NEET 2 project "Radiation-Hardened Circuitry Using Mask-Programmable Analog Arrays" [1]. We present a detailed functional block diagram of the proposed data acquisition system, the thought process leading to technical decisions, the implemented system, and the tested results from the systems. This system will be capable of monitoring at least three parameters of importance to nuclear reactor monitoring: temperature, radiation level, and pressure.

\title{
1. BACKGROUND
}

The reduced availability of truly radiation-hardened semiconductor processes has resulted in development of a variety of techniques to mitigate some of these effects while using standard, readily available commercial processes. Collectively these techniques are referred to as radiation-hardened-by-design or RHBD. These techniques range from actual transistor layout techniques to reduce device damage to the use of special circuit architecture designs ensuring that damaged devices will not be allowed to override the operation of good devices. Radiation-tolerant electronics are therefore one of the major limiting technologies preventing the application of effective telerobotics to high-radiation environments present under severe accident conditions or in support of fuel reprocessing. Moreover, the electronics, once developed, are low cost, enabling frequent replacement when used under high-dose-rate conditions.

This project will develop and demonstrate a general-purpose data acquisition system built from commercial or near-commercial radiation-hard analog arrays and digital arrays that will be the building blocks of a family of future fieldable radiation-hard systems. The outcome will result in the prototype radhard data acquisition system that will be constructed and tested to demonstrate functionality and radhardness of the identified commercially available technology, as applied to a nuclear reactor environment. The system prototype will be delivered along with measured functional metrics for both pre- and postradiation scenarios. Comparison of this data will be performed and will validate the radiation survivability of this technology path. In addition, the measured degradation observed in each of the circuit functions will also be summarized. Successful completion of this project will demonstrate the feasibility of using commercial or near-commercial radiation-hardened custom circuits for this application.

The limiting factor to achieving a radiation-hardened system is the most basic circuits themselves. If the system cannot perform elementary functions accurately in the presence of harsh radiation, it is useless for our application. Existing radiation-hardened designs are available to us in the form of a Triad viaconfigured array (VCA), which includes various operational circuits verified up to $1 \mathrm{Mrad}$. Careful assimilation of these circuits results in the capability to digitize analog data that a sensor would produce. This digital data may then be processed by a controller and transmitted to an end user to assess current reactor environment conditions and, if necessary, a course of action. Accomplishment of this project will include the integration of the Triad rad-hard VCA circuits with temperature, pressure, and radiation sensors, as well as a field-programmable gate array (FPGA) controller, which can be easily substituted for with rad-hard components, into a robust, accurate system. 


\section{PROJECT TASK ORGANIZATION}

The overall project will be divided into four tasks, each of which provides clear objectives and associated metrics for performance evaluation. These are summarized below.

Task 1. Electronics design and hardware selection - A detailed functional block diagram of the proposed data acquisition system will be developed. The level of detail will include the commercial and precommercial parts to be used, the functional interconnect between the various parts, and the overall signal flow and data format definitions. This system will be capable of monitoring at least three parameters of importance to nuclear reactor monitoring: temperature, radiation level, and pressure. Electronic components as well as appropriate sensors will be chosen.

Task 2. Detailed system design and fabrication - Using the analog blocks available in our currently preconfigured VCA, we will perform a detailed schematic design of our system to include the signalprocessing blocks for temperature, radiation, and pressure. Control and data acquisition will be implemented with the Spartan-6 FPGA as well as wired serial communications with a remote computer to be used with this project. In addition, batteries and associated voltage regulators will be selected for powering the system. Fabrication may include a polyimide printed circuit board (PCB) for improved radiation and temperature tolerance. The $\mathrm{PCB}$ will go through a layout process by one of the organizations we utilize for this function and be fabricated by another external vendor. Population of the system board will be performed using one of our in-house technicians or an outside vendor we commonly use. Five prototype systems (sensors, electronics board, battery power supply, and PC serial communications port) will be constructed to support the testing objectives of this work. Quality assurance will be performed at each step by both the submitters and the vendors.

Task 3. System testing and validation (pre- and post- irradiation and temperature) - Evaluation of the performance of the system for both pre- and post-irradiation as well as operation at elevated temperature will be performed. Detailed performance of the system will be documented to ensure the design meets requirements prior to any extended evaluation. A suite of tests will be developed which will allow evaluation before and after irradiation and during temperature. Selection of the radiation exposure facilities will be determined in the early phase of the project. We have used both in-house and external irradiation facilities in the past and have extensive experience in the setup and testing in such facilities. Radiation exposure will consist of a total integrated dose (TID) up to $200 \mathrm{krad}$ or above with several (to be determined) intermediate doses during test. Dose rates will be in various ranges determined by the facility that will be used, but a target maximum will be $20 \mathrm{krad} / \mathrm{hr}$. Many samples of the pre-commercial devices to be used will have been tested in previous projects to doses of at least $300 \mathrm{krad}$ and temperatures up to $125^{\circ} \mathrm{C}$. The complete systems will therefore be tested for performance at intermediate doses and up to the system failure dose. Temperature testing will be performed up to $125^{\circ} \mathrm{C}$, which should be adequate for most target environments [1]. The test suite performed at each test point will consist of operational testing of the three basic measurement functions plus electronic functional testing (power dissipation, voltage offset changes, bandwidth changes, noise variations, etc.). This suite will be developed as part of this task.

Task 4. Data analysis and presentation - Data taken from the pre- and post-radiation/temperature evaluation will be analyzed to quantify variations. Measured performance changes will be identified, and the root cause at the circuit level will be determined. This will give us a good measure of the overall performance of the system that can be clearly communicated to potential users of this technology. 


\section{SCHEDULE/TIMELINE FOR EXECUTING THE PROJECT}

Table 1 shows the timeline associated with each task of the proposed work. These are associated with the milestones and deliverables.

Table 1. Project Timeline

\begin{tabular}{|l|c|c|c|c|c|c|c|c|}
\hline \multirow{2}{*}{ Task } & \multicolumn{3}{c|}{ Year 1 } & \multicolumn{4}{c|}{ Year 2 } \\
\cline { 2 - 9 } & $Q 1$ & $Q 2$ & $Q 3$ & $Q 4$ & $Q 1$ & $Q 2$ & $Q 3$ & $Q 4$ \\
\hline $\begin{array}{l}\text { Task 1. Electronics design and } \\
\text { hardware selection }\end{array}$ & & & & & & & & \\
\hline $\begin{array}{l}\text { Task 2. Detailed system design } \\
\text { and fabrication }\end{array}$ & & & & & & & & \\
\hline $\begin{array}{l}\text { Task 3. System testing and } \\
\text { validation (pre- and post- } \\
\text { radiation and temperature) }\end{array}$ & & & & & & & & \\
\hline $\begin{array}{l}\text { Task 4. Data analysis and } \\
\text { presentation }\end{array}$ & & & & & & & & \\
\hline
\end{tabular}

\section{FUNCTIONAL BLOCK DIAGRAM}

The final goal of the data acquisition system is to provide intelligible data to the end user so that they may assess the current reactor environment operating condition. In order to transform the output signals of the environmental sensors, a few steps must be taken. First, each sensor will output a specific type of signal, and these signals must be converted to a digital structure so a computer processor or controller can process and relay this information to the user in a useful format, as depicted in Fig. 1. The conversion methods for each signal will differ slightly, and those variations can be considered on a lower sublevel.

Final processing of the signals will be performed by the controller to convert the data into a format easily understandable by the viewer, such as temperature, pressure, and radiation count in standard units.

Ultimately, each conversion will result in a final digital number (DN) that the controller will prepare and display for the user. 


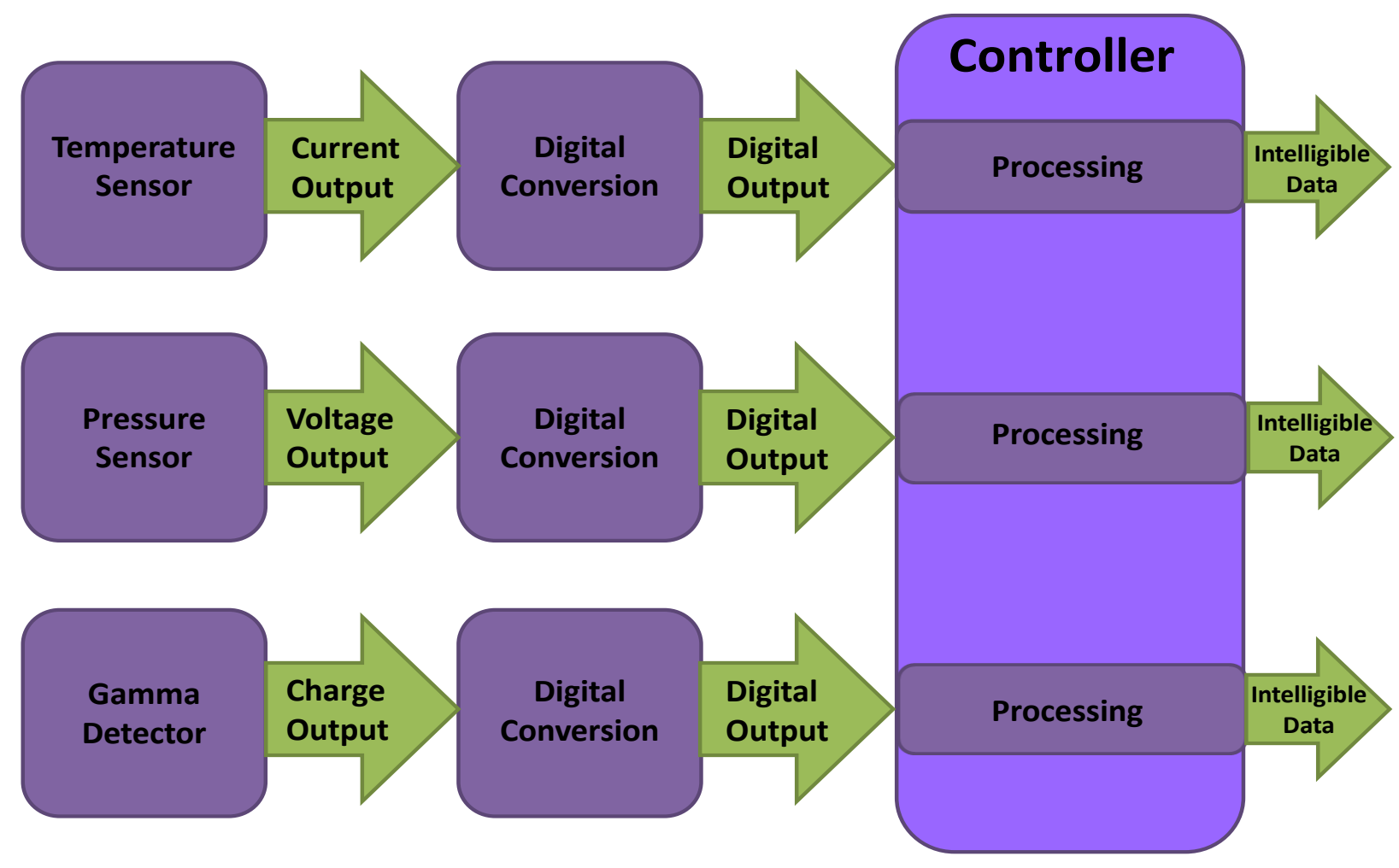

Fig. 1. System functional description diagram.

\section{HIGH-LEVEL ELECTRICAL BLOCK DIAGRAM}

The high-level block diagram, shown in Fig. 2, illustrates the electrical signal-processing paths for three distinct types of sensors. Each sensor will require some level of input gain and filtering and an analog-todigital converter (ADC) to convert the signals to a digital format. The sensor and electronics signal flow will be presented in the following sections. The three channels of signal conditioning/signal processing will be implemented using the circuitry present on the Triad VCA. Each VCA application-specific integrated circuit (ASIC) contains multiple single-ended operational amplifiers, biquad filters designed as input anti-aliasing filters for the sigma-delta modulators which are also on the ASIC, and a bandgap voltage reference.

ASICs are very important to electronic systems for multiple reasons. First, integrated circuits (ICs) have the inherent capability of performing numerous electrical functions and operations within one areaefficient space. Not only do ICs optimize circuit density, but they also offer improved matching behavior performance when compared to using individual functional blocks to accomplish the same operation. This is because the IC fabrication process, although precise, is not perfect, and silicon substrate variations from chip to chip are inevitable. Combining multiple functions into one IC effectively reduces matching errors and provides minimal variation from expected results.

Secondly, as their name implies, ASICs are application specific, which means they can be designed to perform precisely to the exact requirements of an application. On the other hand, general ICs must be designed to satisfy a wide variety of applications, and nothing comes for free with circuit design. Trade- 
offs must be made; thus, certain performance characteristics of the IC will diminish relative to an ASIC. This advantage over general ICs makes ASICs almost necessary, especially when stringent conditions such as extreme environment operation is required.

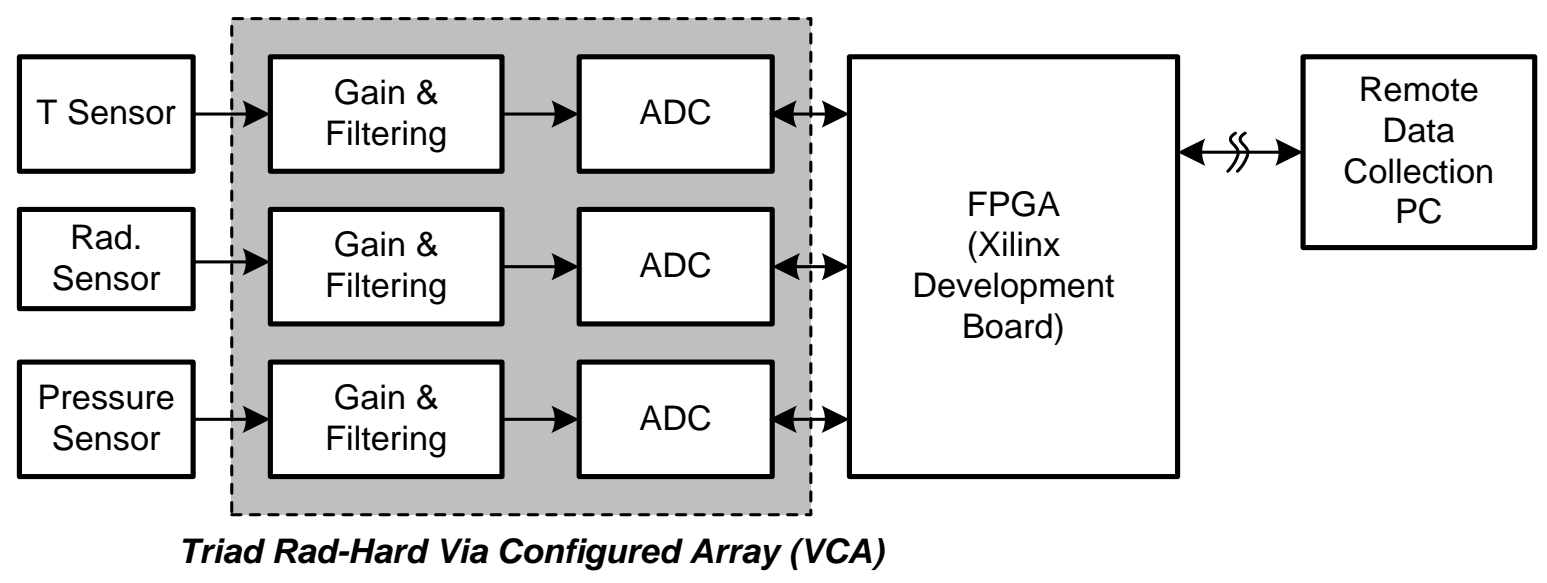

Fig. 2. Data acquisition system based on commercial and near-commercial rad-hard circuits.

As a side note, the stated scope of this proposal is to develop a small data-acquisition demonstration system whose circuitry will be tested for radiation hardness. Development of rad-hard sensors is outside of the scope for this particular proposal; however, the system has been designed so that, with only minor PCB tweaks, existing rad-hard sensors can easily replace their soft counterpart on the sensors board. An obvious follow-on project incorporating rad-hard sensors into this system could be accomplished with appropriate future funding.

\section{SENSORS AND SUPPORT COMPONENT EVALUATION}

\subsection{TEMPERATURE SENSOR}

Within potentially harsh radiation environments such as nuclear reactor sites, ambient temperature can be a significant parameter for monitoring normal and stable reactor operating conditions, as well as detecting early signs of nuclear disaster situations. As a result, accurate and frequent temperature measurements are necessary. For extreme scenarios, these sensors will need to withstand temperature ranges from 0 to $125^{\circ} \mathrm{C}$, TID radiation exposure of at least $300 \mathrm{krad}$, and potential dose rates up to $200 \mathrm{krad} / \mathrm{hr}$ in order to effectively relay data for processing [1]. Although providing extreme environment-hardened sensors was not within the scope of this project, selecting these sensors, budget willing, would go above and beyond expectations.

Throughout the search for temperature sensors, the Intersil ISL71590SEH transducer proved to be a formidable rad-hard solution. Along with its current output, which is useful for application-specific design, the rad-hard sensor boasted a -55 to $125^{\circ} \mathrm{C}$ temperature range and $300 \mathrm{krad}$ total dose operation within $1^{\circ} \mathrm{C}$ of accuracy. The nominal current output value is $298.1 \mu \mathrm{A}$ at $25^{\circ} \mathrm{C}$ (room temperature), and the delta output is $1 \mu \mathrm{A} / \mathrm{K}$. Unfortunately, at a cost of approximately $\$ 325 /$ unit, and with many other components to purchase, it was decided that soft (non-radiation-hardened) sensors would be used.

With this in mind, the ideal solution was to find a sensor matching all of the performance specifications of the Intersil ISL71590SEH, excluding radiation hardness. The Analog Devices AD592 came very close to 
matching these expectations, claiming a -25 to $105^{\circ} \mathrm{C}$ temperature range, $0.5^{\circ} \mathrm{C}$ measurement accuracy, and equivalent current output characteristics. Therefore, the AD592 was selected as our temperaturesensing element. Nearing the conclusion of the project, if sufficient funds remain, a few of the compatible Intersil ISL71590SEH temperature sensors could be purchased and substituted to demonstrate the capability of radiation-hardened sensors within the system.

\subsection{PRESSURE SENSOR}

Much like temperature, pressure is another parameter that can be monitored in reactor environments to reveal ideal or non-ideal operating conditions and also if unsafe conditions may be developing. For harsh environments, we want to measure pressure relative to normal atmospheric pressure, or gauge pressure, up to $2 \mathrm{~atm}$ ( $3 \mathrm{~atm}$ absolute). These pressure ranges are not extreme and do not require high-pressurecapable sensors. Rather, a low-pressure-range, higher-resolution sensor would allow for full-pressurerange swing and the ability to precisely monitor small pressure changes to better fit this application. In addition, we want to establish an output voltage range for the sensor so the analog-to-digital conversion parameters may be easily designed. This can be done with an analog ratiometric pressure sensor, which relates its output voltage proportionally to its input voltage supply and allows for direct analog-to-digital conversion without any pre-conditioning. Since we are designing and constructing PCBs for this system, a board-mount sensor is preferred.

During our initial search for rad-hard sensors, the market was very scarce for pressure sensors in particular. Only one sensor was found that remotely met our requirements, the Omni Instruments Radiation "Resistant" Pressure Sensor. However, the sensor lacked test data to a specific radiation totaldose-tolerance level and was not a board-mount sensor. The Omni Instruments pressure sensor could potentially have been radiation verified locally and implemented within the data acquisition system, but this was not the ideal solution. After relaxing our search requirements to include non-rad-hard sensors, many more options were available, and Honeywell stood out as the leading supplier. Honeywell provides various styles of pressure sensors and combines all of our project needs into one compact, silicon-based sensor with their ASDX Series. This series has multiple custom variations and specifications, such as a variety of pressure ports, pressure ranges, transfer function limits, and supply voltages. For our project, we selected an axial vented port, 30 psi gauge pressure range ( $2 \mathrm{~atm}), 10-90 \%$ calibration, and a $5 \mathrm{~V}$ supply. All of these specifications will suffice for our application, denoting the Honeywell ASDXAV030PGAA5 as our pressure sensor of choice.

\subsection{GAMMA DETECTOR}

Radiation detection is the key parameter in identifying a nuclear reactor disaster scenario. Radiation will escape the reactor containment before ambient temperature or pressure changes are realizable. Among the most prevalent forms of radiation detection are ionization chambers, proportional detectors, and GeigerMüeller (G-M) tubes, which are all based on the effects of charged particles passing through a gas [2]. All three of these methods can be used for our application. However, ion chambers and proportional detectors both output relatively small electrical signals which require amplification. Their upside is that they can resolve higher dose rates than a G-M tube. The G-M tube achieves inherent amplification through the use of large electric potential biasing $(\sim 500 \mathrm{~V})$, which results in an intense "avalanche effect," causing ion pairs freed by high-energy gamma radiation to collide and trigger additional avalanches during their paths toward the electrodes. Although this produces an amplified charge output, the additional particle collisions slow down transit speed of the charge carriers and effectively increase total time from initial excitation to final output. As a result, a G-M tube exhibits a large dead time, or length of time for which the G-M tube is inoperative, because the tube needs to reset itself by evacuating all charge carriers from the gas chamber. The positive charge carriers, or holes, are heavier and inherently move much slower 
than the electrons and thus take longer to evacuate. Because the G-M tubes generate an increased number of these ion pairs, they take longer to reset, which limits their high-dose-rate resolution capability.

Nuclear applications beyond design basis can require a dose rate resolution of up to $200 \mathrm{krad} / \mathrm{hr}$. G-M tubes can only resolve, at maximum, about $1 \mathrm{krad} / \mathrm{hr}$. While ion chambers and proportional counters can measure much higher dose rates, they complicate the interfacing circuitry with the need for low-noise amplification. In addition, reliable G-M tubes are much more accessible and cost efficient than their counterpart. For simplicity of signal interface design and cost, an LND 714 G-M tube will be used as our gamma-radiation-sensing element. In order to address the inherently low-dose-rate resolution of approximately $100 \mathrm{rad} / \mathrm{hr}$ for the LND 714, we will shield the tube. This technique will reduce the radiation dose incident on the G-M tube by a certain factor, which we will characterize for accuracy, and will result in a higher effective-dose-rate capability for the G-M tube.

\subsection{SUPPORT COMPONENTS}

Some additional components are necessary to provide the power required by the system and enable full circuit operation. All circuits within the system will need a specific level of voltage and current supply generated from a voltage regulator, and for radiation-rich environments, this supply must be very robust across a large range of dose rates, potentially up to $200 \mathrm{krad} / \mathrm{hr}$. Microsemi produces a variety of radiation-hardened voltage regulators with differing total dose capabilities, but the SGR117A model stood out with the highest TID capability of all, claiming total doses exceeding $1 \mathrm{Mrad}$. This voltage regulator can produce output voltages ranging from 1.25 to $34 \mathrm{~V}$, supply at least $1.5 \mathrm{~A}$ of current across all operating conditions, and maintain a $0.3 \%$ load regulation specification. The SGR117A is available in a 3 -pin $\mathrm{K}$ package with a thermal dependence of only $3^{\circ} \mathrm{C} / \mathrm{W}$ and operating temperatures up to $150^{\circ} \mathrm{C}$. These voltage regulator specifications can satisfy power requirements for every circuit within the system with the exception of the G-M tube, which will require a high-voltage DC converter to reach a $500 \mathrm{~V}$ potential.

The LND 714 G-M tube recommends a $500 \mathrm{~V}$ potential for operation to capture high-speed freed electrons and holes but does not require significant current supply because the charge produced comes directly from the tube itself. EMCO produces numerous high-quality, high-voltage DC converters for avalanche photodiode and photomultiplier tube applications, which is very similar to and would be ideal for our system. The EMCO A05P5 1W positive adjustable DC converter can output up to $500 \mathrm{~V}$ at a maximum current of $2 \mathrm{~A}$, with only a $5 \mathrm{~V}$ and less than $200 \mathrm{~mA}$ input at no load. This EMCO DC converter is not radiation hardened and will be placed on the shielded sensor's board to minimize highpower transmission lengths and optimize board density. The $5 \mathrm{~V}, 200 \mathrm{~mA}$ converter input will be supplied by the Microsemi voltage regulator from the radiation-hardened board. High-current power will need to propagate across multiple PCBs; however, twisted pairs can be used to transmit this power with low losses and minimal interference.

\section{TRIAD RAD-HARD ASIC CAPABILITIES}

\subsection{OPERATIONAL AMPLIFIER}

For data acquisition applications, operational amps (opamps) play an important role in the processing of analog data. Opamps are gain blocks that are designed to provide high-voltage gain and negative feedback in order to implement controlled-gain functions as well as signal buffering and filtering. Opamps have been around in various forms since the 1950s and are ubiquitous in today's electronic devices, being found in almost any circuit that has any analog functionality. For this project, these op amps will be used within the sigma-delta modulators for analog-to-digital conversion of temperature and pressure data, as well as being used as a comparator for verification of gamma radiation counts. 
The Triad rad-hard ASIC [3] contains various RHBD circuits commonly used in analog circuit design, with specific alterations to each individual block, allowing for increased application versatility. The first circuit is fundamental to any analog system - the operational amplifier. The chip contains several different types of operational amplifiers, all of which are considered proprietary. We will not be discussing the specifics of the design characteristics of the amplifiers in this and future reports because of their proprietary nature.

\subsection{SIGMA-DELTA MODULATOR}

Highly important to any modern data acquisition system is the ability to precisely digitize sensor data at acceptable rates. The Triad rad-hard ASIC includes a second-order, sigma-delta modulator that can be used as an ADC. Like the operational amplifiers, the sigma-delta modulators are also proprietary. We will therefore not be discussing the specifics of the design characteristics of the modulators in this and future reports because of their proprietary nature.

The digital output is a pulse-density-modulated bit stream that will be digitized with digital sinc filtering [4]. Each of the sigma-delta modulators were implemented with their respective operational amplifiers.

\subsubsection{Analog-to-Digital Converter Introduction}

An ADC converts an analog signal composed of a continuum of values to a digital result [4]. The ADC output is a digital "best" approximation of its input signal, with the approximation error (or quantization error) being related to the ADC resolution. ADCs can be classified as one of two types: Nyquist rate or oversampling. Nyquist rate converters sample the input signal at approximately twice the highest frequency of the input signal using a number of different architectures including successive approximation, pipeline, algorithmic, and flash. Oversampling converters achieve improved resolution at the expense of conversion speed. In oversampling converters, improved performance is accomplished by sampling the input signal at rates much higher than the Nyquist rate and shaping the quantization noise using a noise-shaping filter.

Since an ADC produces a digital approximation of an analog input signal, every ADC operation produces error, known as quantization error. Typically, the quantization noise has a flat power spectral density so that the total noise increases as the bandwidth increases (or sample rate increases). This becomes a limiting factor in the realization of very high-resolution ADCs.

The sigma delta ADC architecture uses two techniques to improve the signal-to-noise ratio: oversampling and noise shaping. By oversampling, the quantization noise power is spread out over a larger bandwidth, resulting in reduced integrated noise in the signal bandwidth. In addition to oversampling, noise shaping is also accomplished in the modulator, which significantly reduces the integrated noise in the signal bandwidth, depending on the order of noise shaping. The effect of noise shaping is graphically demonstrated in Fig. 3 for unshaped quantization noise up to fourth-order noise shaping. One can see the significant reduction in noise at the lower left side of the plot where the signal bandwidth of interest is located. 


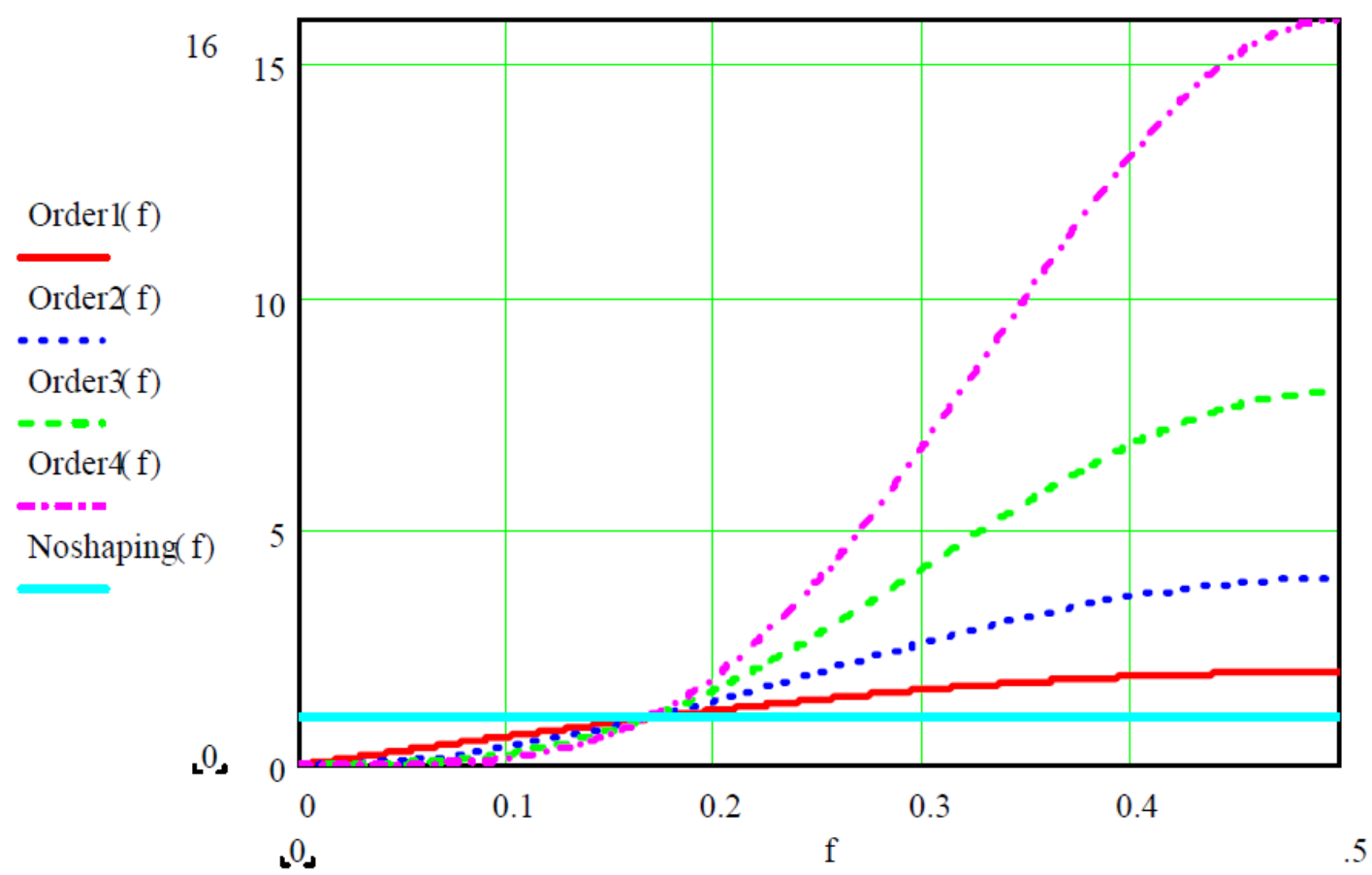

Fig. 3. Noise shaping to move much of the quantization noise out of the signal band.

Oversampling of the input signal further distributes the shaped noise as the peak of the noise distribution is stretched to the new sampling frequency/2 $\left(\mathrm{f}_{\mathrm{s}} / 2\right)$, resulting in a reduced noise power in the signal bandwidth of interest. This is demonstrated graphically in Fig. 4.
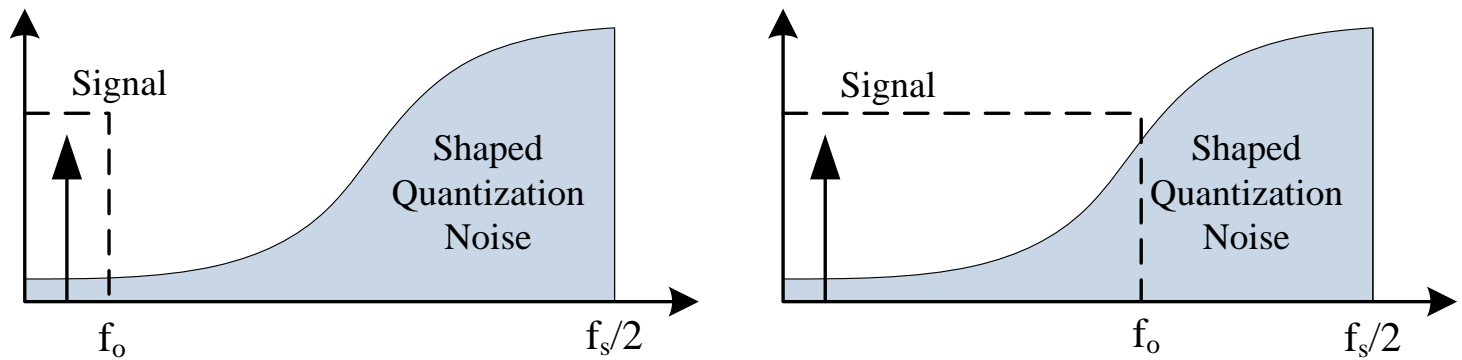

Fig. 4. Effect of quantization noise shaping and oversampling on the in-band noise in a sigma-delta ADC. The left plot shows a higher oversampling ratio than shown on the right. The signal bandwidth is marked with dashed lines.

Using a combination of these two noise reduction methods allows for a significant improvement in the theoretical resolution of the ADC. Figure 5 shows this effect as a function of oversampling ratio $\left(M=f_{s} / f_{o}\right)$ and the quantization noise-shaping order (or modulator order, L). Specific values for these curves are summarized in Table 2. 


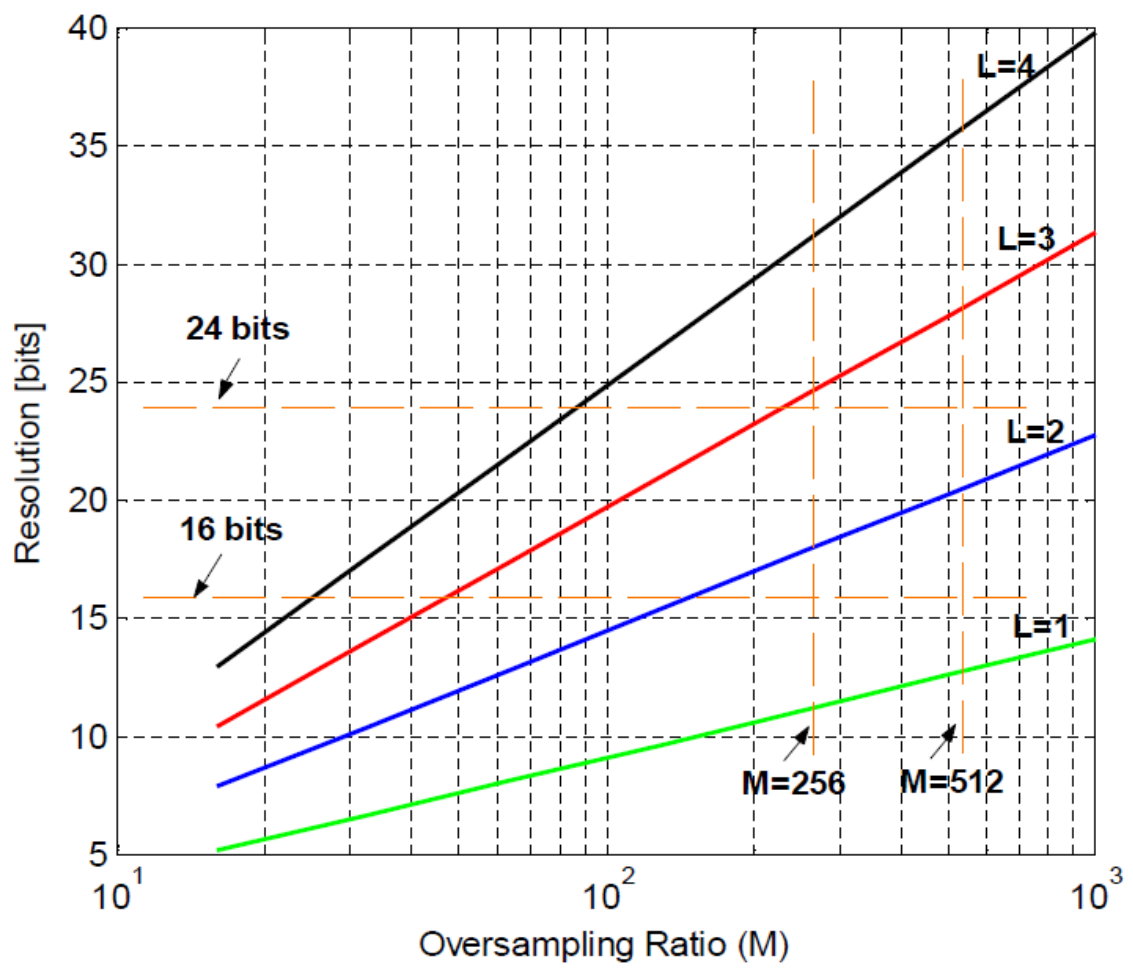

Fig. 5. Theoretical ADC resolution as a function of oversampling ratio and noise-shaping order.

Table 2. Theoretical sigma-delta ADC resolution as a function of modulator order (L) and oversampling ratio (M)

\begin{tabular}{c|c|c|c|c}
\hline $\begin{array}{c}\text { Modulator } \\
\text { Order }\end{array}$ & $\mathbf{M = 6 4}$ & $\mathbf{M = 1 2 8}$ & $\mathbf{M}=\mathbf{2 5 6}$ & $\mathbf{M}=\mathbf{5 1 2}$ \\
\hline 1 & 8.14 & 9.64 & 11.14 & 12.64 \\
\hline 2 & 12.86 & 15.36 & 17.86 & 20.36 \\
\hline 3 & 17.45 & 20.95 & 24.45 & 27.95 \\
\hline 4 & 21.98 & 26.48 & 30.98 & 35.48 \\
\hline 5 & 26.47 & 31.97 & 37.47 & 42.97 \\
\hline
\end{tabular}

Following noise shaping and oversampling, the modulator output is digitally filtered and decimated to remove the out of band noise and downsample the signal back to the desired digital output rate $\left(\mathrm{f}_{\mathrm{o}}\right)$. This is graphically demonstrated in Fig. 6. 


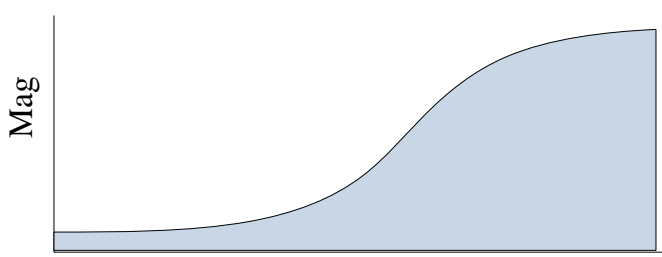

Frequency

Oversampling \& Noise Shaping

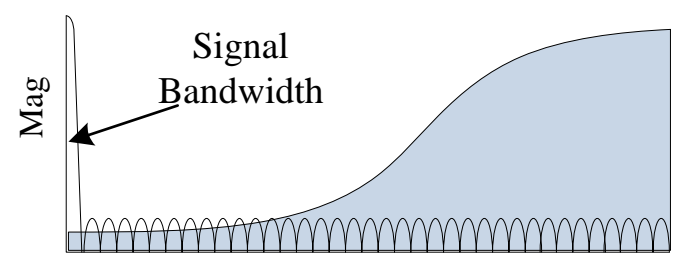

Frequency

Digital Filtering

Fig. 6. Digital filtering of the signal bandwidth following oversampling and noise shaping.

The actual implementation of the sigma-delta ADC is composed of two primary modules, the modulator and the digital filter (Fig. 7). Note that the input is an analog signal, the modulator is an encoded bit stream, and the output of the digital filter is the digitized output signal. An example sigma-delta modulator implemented using switched capacitor circuits and linear amplifiers is shown in Fig. 8 (secondorder noise shaping). This represents one of the more straightforward, low-shaping-order designs. Digital filters can be implemented using a number of different filter topologies. A simple example, similar to the topology that will be implemented in the FPGA for this program, is shown in Fig. 9. This sinc filter topology provides reasonable performance and can be implemented using simple shift registers and adders.

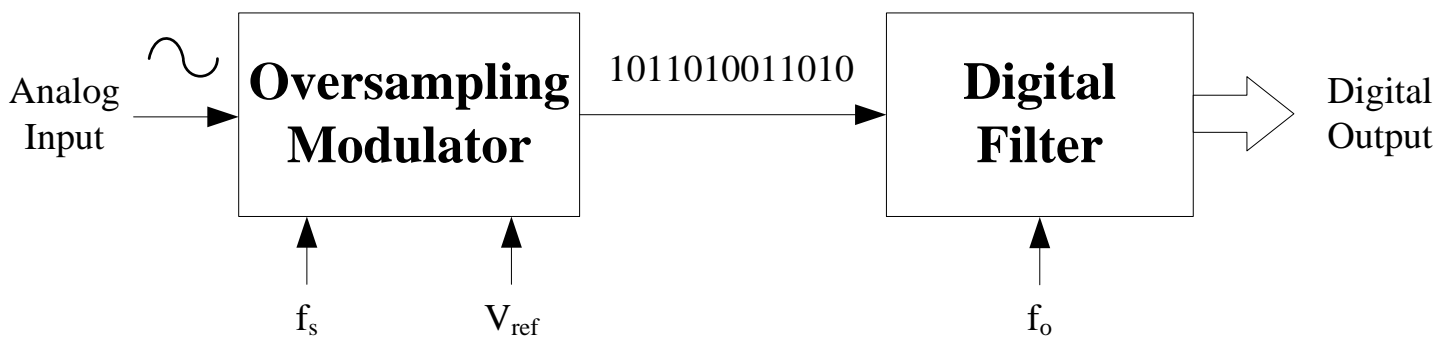

Fig. 7. Sigma-delta ADC block diagram.

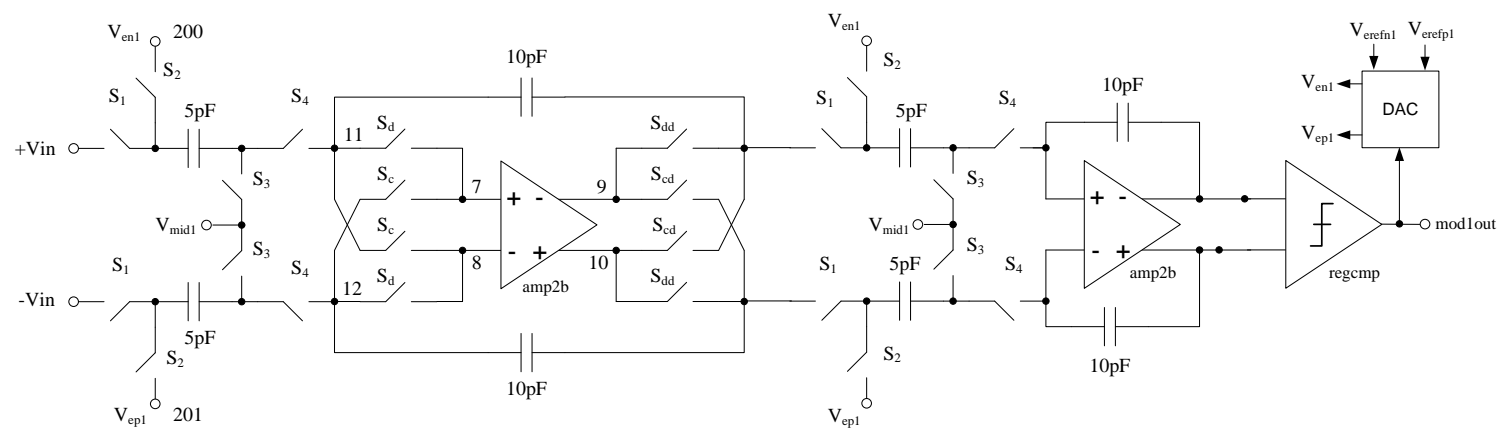

Fig. 8. Second-order sigma-delta modulator implementation using switched capacitor circuits. 


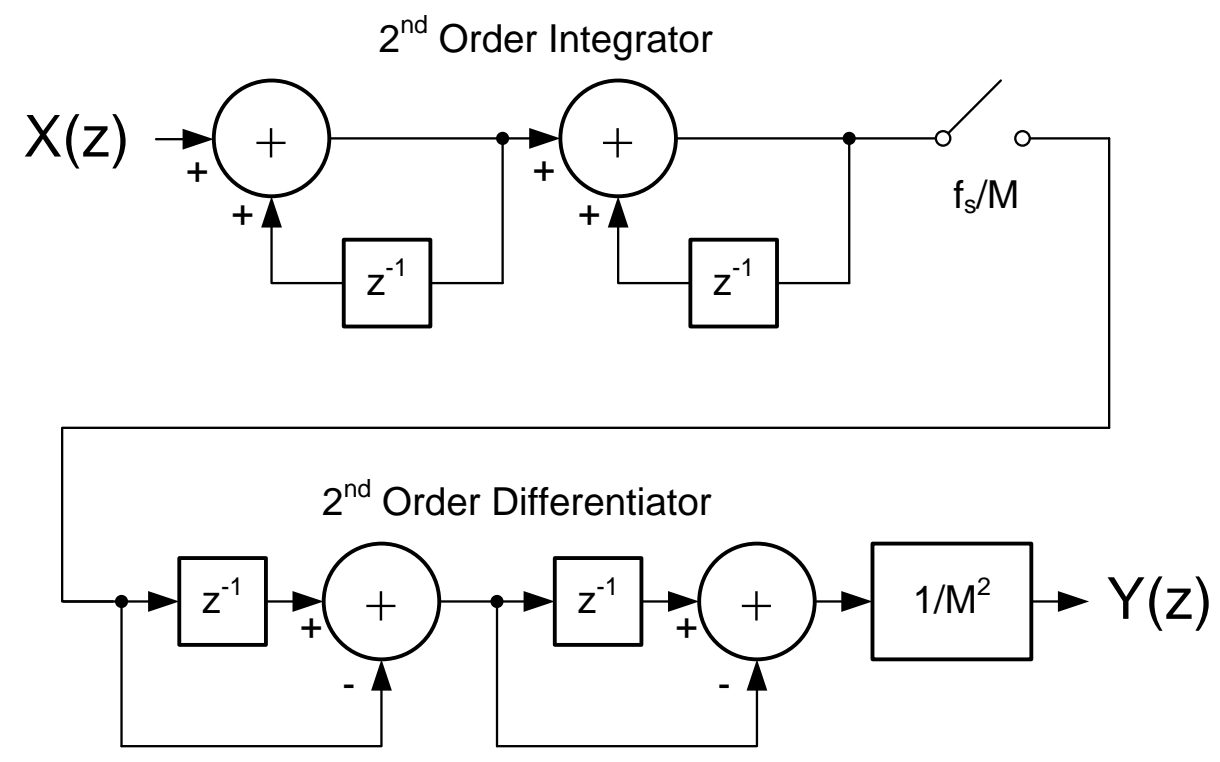

Fig. 9. Second-order sinc filter topology (with decimation).

\section{DIGITAL CONTROLLER SEARCH}

The digital control module handles the control and sequencing of all instrument functions including data acquisition, data processing and packetizing, command interpretation and execution, and data and command communication. The design approach selected for this program is to use a programmable device for the digital controller to enable iterative development of the system without the need to replace hardware modules. Common programmable hardware devices considered for this application include micro-controllers, FPGAs, and complex programmable logic devices (CPLDs). Given the control functions required for this application (state machine sequencing, simple digital filtering, data packet generation, command execution, and serial communications), FPGAs were selected over the other candidates as the optimum target device architecture for the digital controller in this development.

A study was carried out to assess the commercial availability of rad-hard or rad-tolerant FPGAs. The findings of this study, as summarized in Table 3, indicate that only a very limited number of commercial FPGA products are available for applications requiring rad-hard $(\geq 1 \mathrm{Mrad})$ or rad-tolerant $(\geq 300 \mathrm{krad})$ operation. Note that these products allow true in-field programmability, thus accommodating anticipated iterative design revisions. In addition to those shown in Table 3, many other commercial programmable logic solutions were also considered that are based on anti-fuse technology but were deemed unsuitable since only a single programming of the device is allowed. Also shown in Table 3 are the availability and cost of reprogrammable devices. For FPGAs having $\geq 300 \mathrm{krad}$ TID, all components were out of stock with no availability until 2015. Due to the very high cost and long delivery times, the rad-hard/radtolerant commercial FPGAs will not be used in the prototype system developed in this program. 
Table 3. Comparison of commercially available rad-hard or rad-tolerant FPGAs

\begin{tabular}{ccccc}
\hline Manufacturer & Family & TID $(\mathbf{r a d})$ & $\begin{array}{c}\text { Gates } \\
\text { *Logic Cells }\end{array}$ & Availability/Unit Cost \\
\hline Xilinx & Virtex-4QV & $>300 \mathrm{k}$ & $>200 \mathrm{k} *$ & $2015, ?$ \\
Xilinx & Virtex-5QV & $>1 \mathrm{M}$ & $131,072^{*}$ & $2015, \sim \$ 80 \mathrm{k}$ \\
Atmel & AT40KEL & $300 \mathrm{k}$ & $46 \mathrm{k}$ & No stock found \\
Atmel & ATF280 & $300 \mathrm{k}$ & $280 \mathrm{k}$ & No stock found \\
Microsemi & RT ProASIC3 & $>100 \mathrm{k}$ & $\leq 3 \mathrm{Meg}$ & Now, $\$ 8 \mathrm{k}$ \\
\hline
\end{tabular}

After determining the degree of difficulty and cost associated with acquiring rad-hard FPGAs, the approach selected for this program was to use a commercially available "soft" FPGA or CPLD, a less complex and cost-programmable logic device. This approach will maintain the full functionality of the system, allow re-programmability, is low in cost, and has strong industry support. Specifically, two Xilinx family devices were chosen: the Spartan 6 FPGA and the CoolRunner II CPLD. Both of these device families have low-cost development systems (includes a single board with device and development tools/software) and are well supported by the manufacturer. A further advantage of using these Xilinx devices over other commercial equivalents is the migration path to rad-hard Xilinx Virtex family devices (Virtex-4QV and Virtex-5QV) is likely easier. We will begin the digital controller design using the CoolRunner II CPLDs. If this family does not have sufficient resources, then we will quickly port the design to the more complex Spartan 6 FPGA. Development kits have been procured for both Xilinx devices (Fig. 10). Both units provide a universal serial bus (USB) interface, switches, and various other resources for adding peripherals to aid in system development.

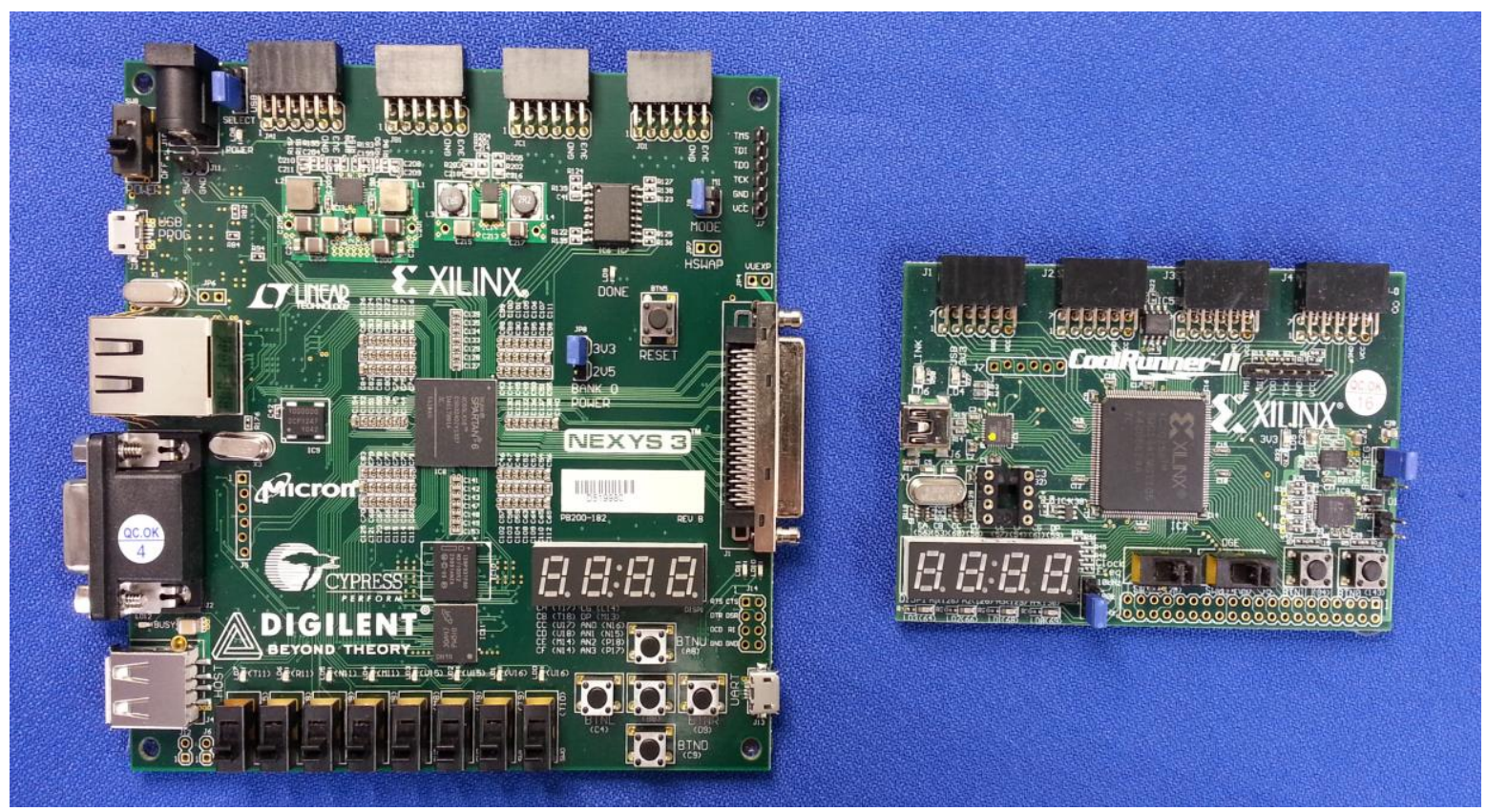

Fig. 10. Development kits purchased for the digital controller development: Digilent Nexys-3 Spartan-6 XC6SLX16-CS324 (left) and Xilinx CoolRunner-II Evaluation Board with XC256-7TQG144C (right). 


\section{DESIGN}

Because of the unavailability of affordable rad-hard FPGA controllers, the data acquisition system design must be split into three board designs. One board will support the temperature and pressure sensors, as well as the gamma detector, but will not be directly irradiated due to the presence of soft sensing components. However, this board will be placed within certain proximity of the radiation source during testing so that some level of gamma events can be counted, while avoiding damage to soft components. Another board, which houses the entire radiation-hardened analog signal conditioning circuitry, will be directly irradiated by a gamma source for proof of concept. A third board will provide processing and control for the system through the use of the soft FPGA. This board will not be irradiated at any level but will be designed so that future work toward a radiation-hardened controller would be easily interchangeable with the current interface. This setup will satisfy the requirements of the radiationhardened data acquisition system project, considering all components within the system that are not radiation tolerant are commercial or pre-commercial parts that can be easily replaced with a sufficient budget.

\subsection{SENSORS SIGNAL CONDITIONING AND BLOCK FLOW}

As aforementioned, the sensors board will provide temperature, pressure, and gamma radiation detection elements. For each of these nuclear environment critical measurements, data is converted to electrical form by the sensors and needs conditioning so that they may be digitized. Specifically, voltage waveforms dependent on their respective environment parameters, as seen in Fig. 11, are required for the available Triad ADCs. The Analog Devices AD592 temperature sensor outputs a nominal current at room temperature $\left(25^{\circ} \mathrm{C}\right)$ of $298.1 \mu \mathrm{A}$ and will vary at $1 \mu \mathrm{A} / \mathrm{K}$. The sensor also requires a supply voltage between 4 and $33 \mathrm{~V}$, which will be provided by the selected Microsemi rad-hard voltage regulator. Placing a $10 \mathrm{k} \Omega$ resistor at the sensor output converts this current into a DC voltage of $2.981 \mathrm{~V}$ with a variance of $10 \mathrm{mV} / \mathrm{K}$. This resistance value optimizes the $\mathrm{V}_{\text {out,DC }}$ and $\Delta \mathrm{V}$ components of the temperaturesensing circuit output voltage $\mathrm{V}_{\text {out }}$ so that the common voltage of the ADC is not exceeded and temperature change resolution is measureable. This voltage is passed to rad-hard block sigma-delta modulator for digitization.

The Honeywell ASDX pressure sensor previously selected outputs a voltage dependent on gauge pressure and proportional to supply voltage. Based on the sensor characteristics, such as $5 \mathrm{~V}$ supply and 10-90\% calibration, the output voltage will be $0.5 \mathrm{~V}$ minimum plus $1.66 \mathrm{~V} / \mathrm{atm}$ above the sealed $1 \mathrm{~atm}$ reference pressure, up to $2 \mathrm{~atm}$, as seen in the detailed sensors block diagram in Fig. 11. These voltage ranges are sufficient for analog-to-digital conversion without signal conditioning; therefore, this voltage is connected directly to the Triad sigma-delta modulator circuit input.

The LND 714 G-M tube is a purely gamma radiation detection device and requires a large electric field, in the range of $500 \mathrm{~V}$, in order to gather quickly moving electron-hole pairs freed by high-energy incident radiation. When a radiation event occurs, the G-M tube essentially "shorts" as a result of numerous charge carriers traveling toward their respective electrodes. Sizeable current-limiting resistors are required to prevent any significant current spikes as a consequence of shorting a $500 \mathrm{~V}$ electric field, as shown in Fig. 11. As the G-M tube shorts, electrons move toward the positive supply, producing a fast positive current spike at the cathode. Since this waveform has a very high-frequency AC characteristic, it will take the low impedance path through the capacitor and be converted to voltage by the potentiometer. This voltage is then passed to the rad-hard comparator for event validation. Since it is unclear how much charge will be converted and gathered with each radiation event, characterization of the G-M tube and potentiometer, and thus the output voltage, will need to be done before accurate output behavior can be stated. 


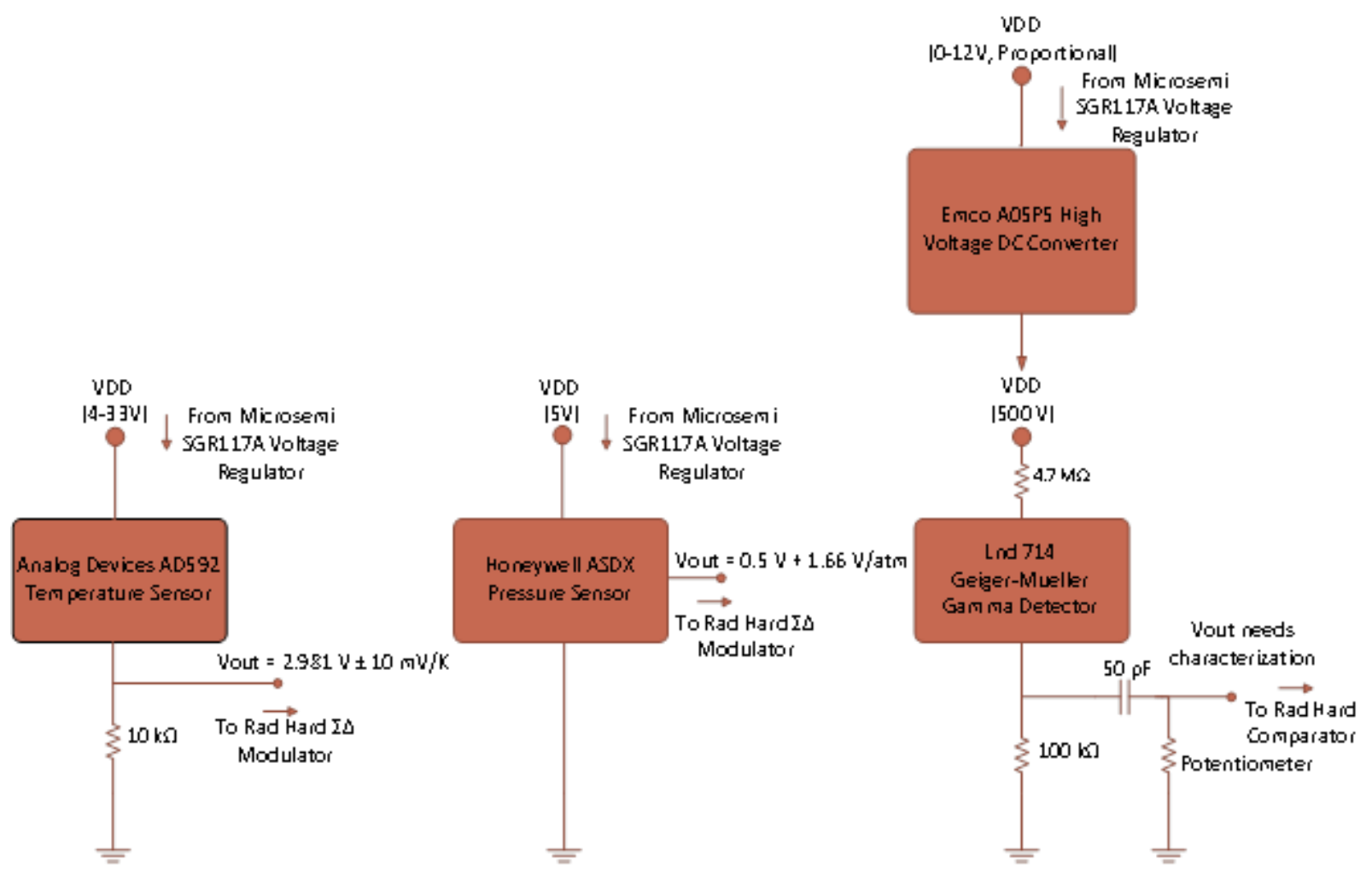

Fig. 11. Detailed sensor block diagram.

\subsection{RADIATION-HARDENED ANALOG BLOCK FLOW}

The temperature, pressure, and gamma radiation sensors will supply voltage waveforms to radiationhardened analog circuits on the Triad chip to begin analog-to-digital conversion. Since these measurements are inherently low frequency in nature, low-frequency single-ended op amps, and thus lowfrequency single-ended sigma-delta modulators, will be chosen from the Triad chip selection in order to increase noise filtering and reduce power consumption.

Both the temperature and pressure sensor analog data will undergo sigma-delta modulation to generate a digital pulse-density-modulated bit stream. This bit stream will then be converted into a DN through proper filtering. The sigma-delta modulator works by increasing the output bit-stream pulse density as the input analog waveform voltage increases and decreasing the bit-stream pulse density as the input analog waveform voltage decreases. Given a stable loop, the output bit streams are passed to the FPGA digital sinc filter for final translation into a $D N$, as seen in Fig. 12. Different values of $V_{c m}, V_{\text {refp }}$, and $V_{\text {refn }}$ for sigma-delta modulation will be needed for their respective temperature and pressure data, considering these waveforms exhibit differing peak voltages and DC offsets.

Digitizing radiation events is much different from analog waveforms in that the charge generated by the event is only present for a short period of time and needs to be classified. The current spike output from the G-M tube is converted to voltage through a resistor, and depending on the peak level of current, the voltage will also reach a certain peak value. In order to discern whether a voltage spike is in fact a radiation event, a certain voltage threshold must be met. This can be achieved by comparing the voltage spike with a reference voltage that is unachievable by any excitation other than a radiation event. If the voltage spike surpasses this threshold, the comparator will output a digital "high" value, representing one event, and subsequently return to a digital low in preparation for the next event. These events can be summed with an FPGA digitally implemented counter, which will notify users of the TID, as well as the 
dose rate, which is derived through the rate of change of the event count. Because of the absence of a comparator circuit on the Triad chip, the output of an op amp will need to be buffered so as to replicate a comparator and produce a full-swing digital output that can be counted.

Two other components will be present on the rad-hard PCB - the Microsemi voltage regulator and the Frequency Management International (FMI) frequency synthesizer. The rad-hard voltage regulator, which is radiation hardened beyond 1 Mrad TID, will supply the voltage and current necessary for all circuits to operate and will itself be powered by a lead acid battery. Multiple copies of the voltage regulator will be present for circuits with varying voltage requirements. The rad-hard frequency synthesizer is capable of precise operation to at least $300 \mathrm{krad}$ TID and will serve as the clock generator for the FPGA controller.

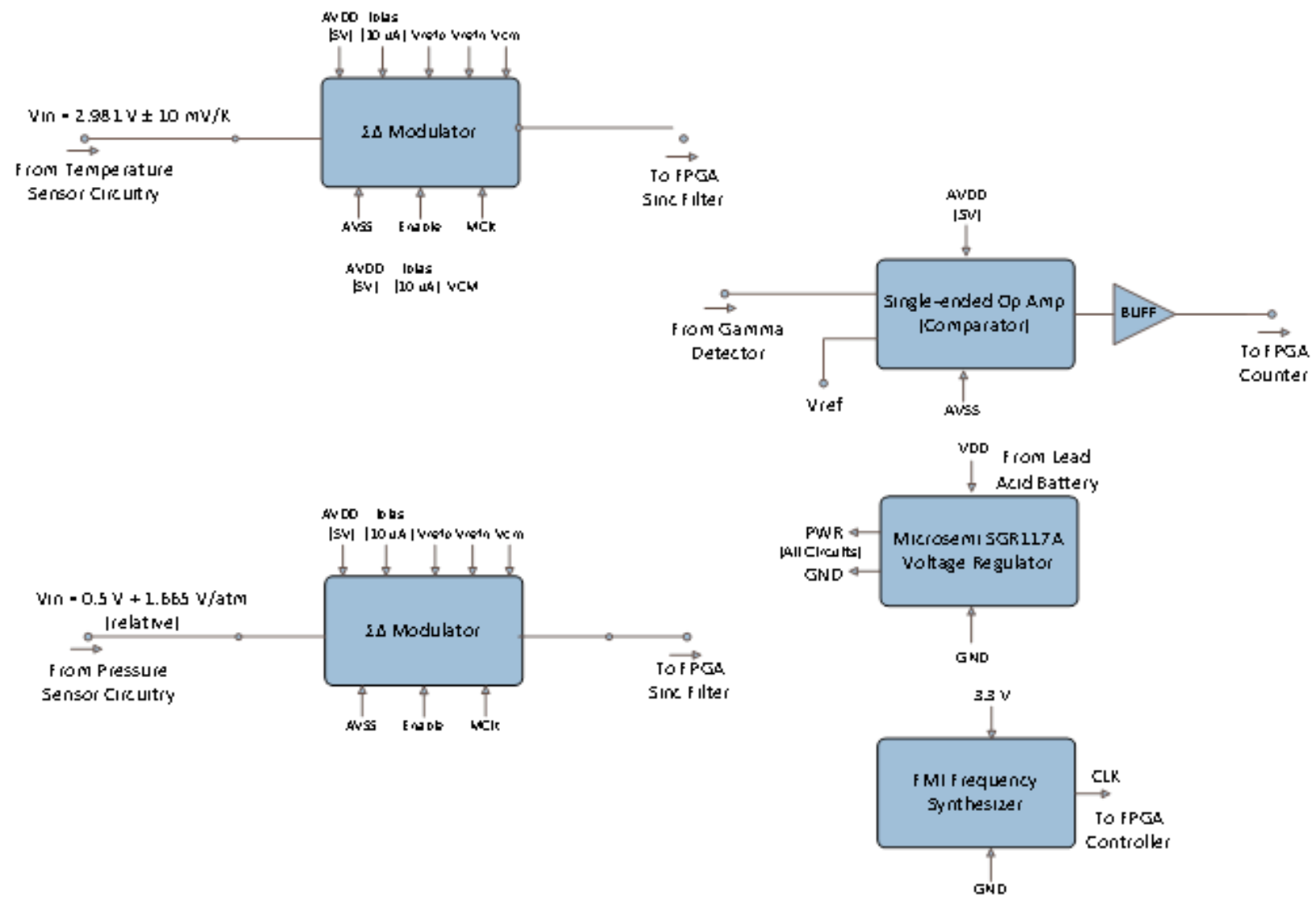

Fig. 12. Detailed radiation-hardened block diagram.

\subsection{NON-RADIATION-HARDENED CONTROL BLOCK FLOW}

The final stages of data analog-to-digital conversion will be implemented digitally with the soft Xilinx Spartan 6 FPGA. Bit-stream data output from the temperature and pressure data circuitry will need sinc filtering to produce a DN, while gamma radiation event data requires a counter to sum each event and report a continuous DN count. Both the sinc filter and counter will be programmed to accurately condition their respective signals into a final digital format. This digital data will then be converted to serial format and transmitted via RS-232 to a remote PC for observation, as shown in Fig. 13. 
Some components from the rad-hard board (RHB), such as the sigma-delta modulator and bi-quad filter, require certain digital control and clock signals to enable proper operation. These signals will also be precisely timed and managed by the FPGA. All blocks, control signals, and external communication will be designed and coded accordingly using either FPGA HDL language, VHDL, or Verilog.

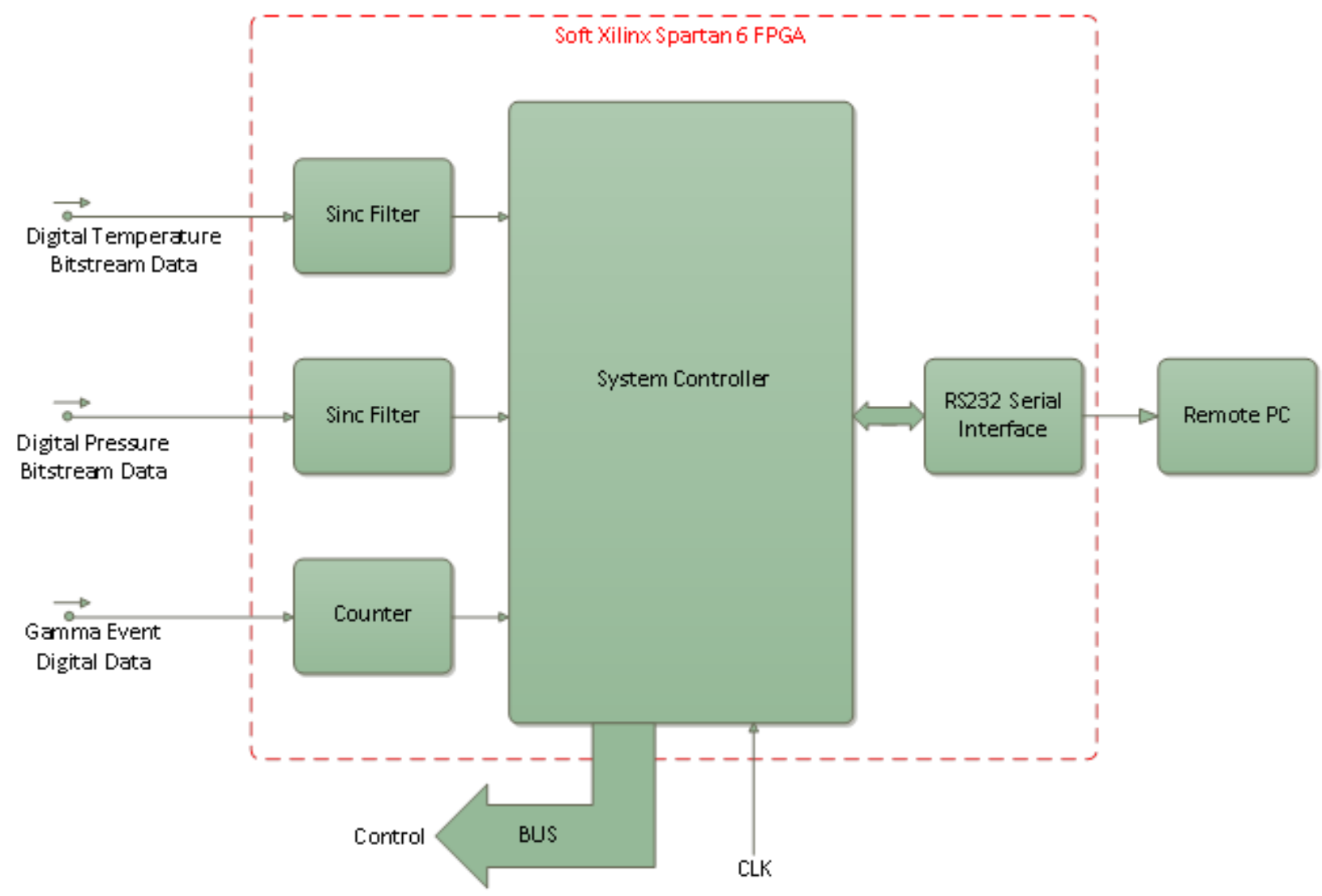

Fig. 13. Detailed Soft (non-rad hard) Block Diagram.

\section{SYSTEM DEVELOPMENT}

The system was developed and partitioned as shown in Fig. 14. The boards labeled sensor board, RHB, and interface board (IB) were designed at Oak Ridge National Laboratory (ORNL). The Nexys 3 board is the commercial board developed and sold by Digilent, Inc., that contains the Spartan 6 FPGA, along with the communications hardware interface. Of all the boards shown, only the RHB has hardened components. The other boards were not designed to be exposed to radiation. The system, with the exception of the commercial Nexys 3 board, is designed to operate off 9-12 V DC so that either nickel metal hydride $(\mathrm{NiMH})$ batteries or lithium batteries can be used. The Nexys 3 board actually uses a $110 \mathrm{~V}$ to $5 \mathrm{~V}$ DC universal adapter and was not redesigned to be used with a battery, but this could be done if required. If, after surveying the radiation test facilities, it is determined that a battery for this board is necessary, one will be provided. The board developments and associated software will be individually presented. 


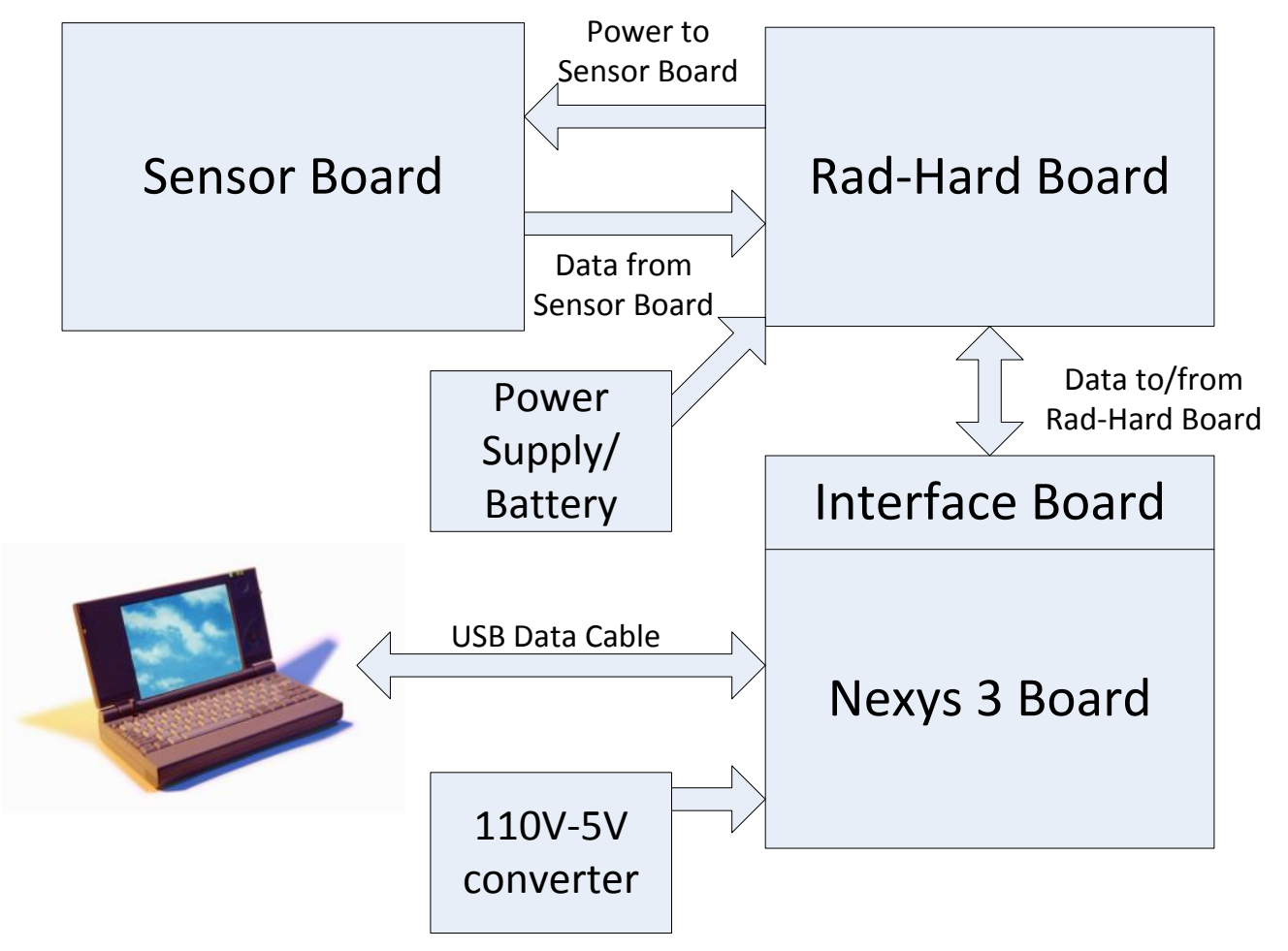

Fig. 14. System partition and function.

\subsection{RAD-HARD BOARD}

The RHB consists of the Triad ASIC; the FMI synthesizer ASIC; and various passive resistors, capacitors, and connectors used for board-board or board-power connections. As previously mentioned, the RHB implements the interface functions between the sensors and the ADCs, and the ADCs themselves. It also contains the rad-hard voltage regulators which supply $5 \mathrm{~V}$ and $3.3 \mathrm{~V}$ to the on-board circuitry.

The temperature, pressure, and gamma radiation sensors will supply voltage waveforms to radiationhardened analog circuits on the Triad chip to begin analog-to-digital conversion. Since these measurements are inherently low frequency in nature, low-frequency single-ended op amps, and thus lowfrequency single-ended sigma-delta modulators, will be chosen from the Triad chip selection in order to increase noise filtering and reduce power consumption.

Both the temperature and pressure sensor analog data will undergo sigma-delta modulation to generate a digital pulse-density-modulated bit stream. This bit stream will then be converted into a DN through proper filtering. The sigma-delta modulator works by increasing the output bit-stream pulse density as the input analog waveform voltage increases and decreasing the bit-stream pulse density as the input analog waveform voltage decreases. Given a stable loop, the output bit streams are passed to the FPGA digital sinc filter for final translation into a DN, as seen in Fig. 15. Different values of external offsets for sigmadelta modulation are needed for their respective temperature and pressure data, considering these waveforms exhibit differing peak voltages and DC offsets. 


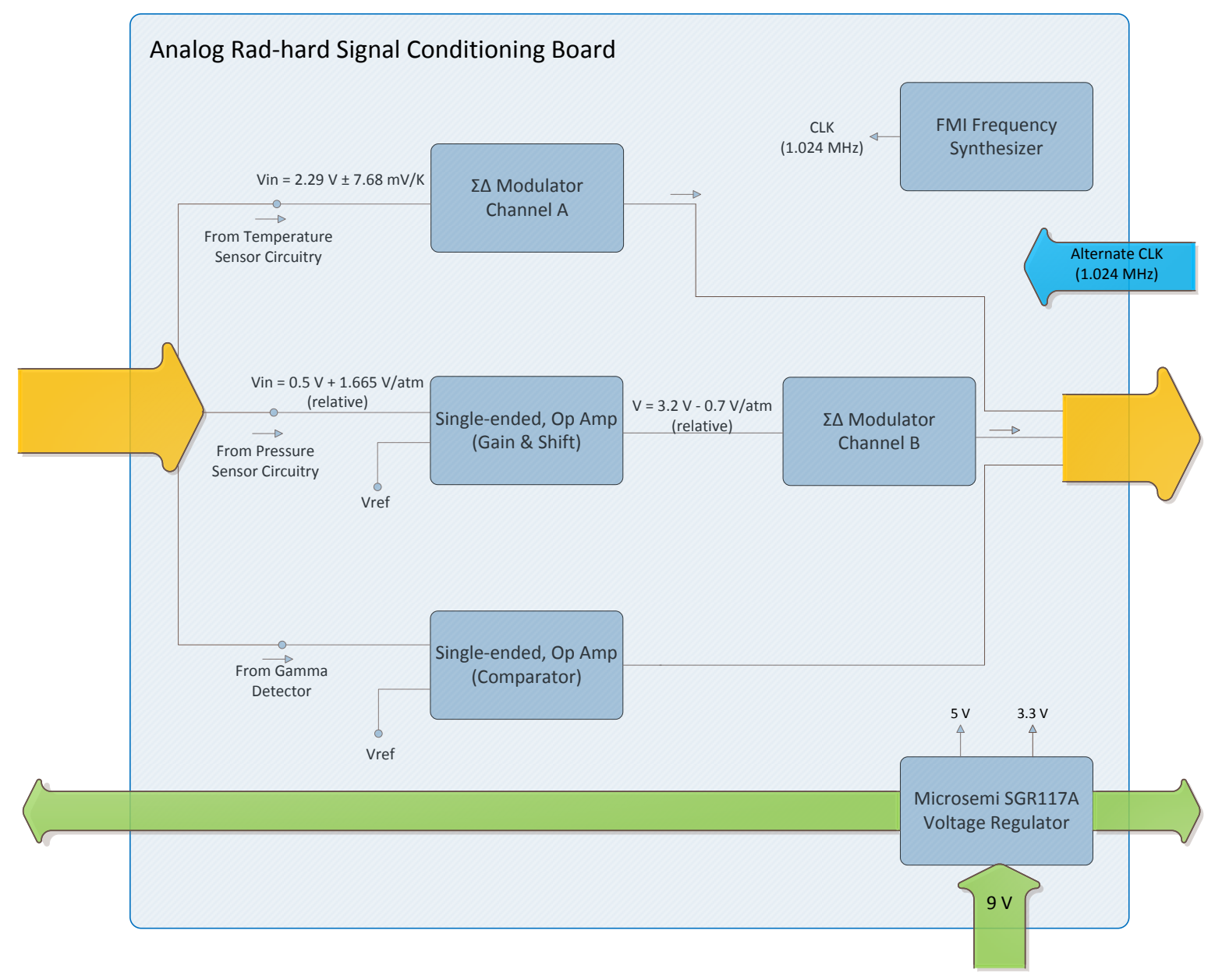

Fig. 15. Detailed radiation-hardened block diagram.

Digitizing radiation events is much different from digitizing analog waveforms in that the charge generated by the event is only present for a short period of time, and it needs to be classified. The current spike output from the G-M tube is converted to voltage through a resistor, and depending on the peak level of current, the voltage will also reach a certain peak value. In order to discern whether a voltage spike is in fact a radiation event, a certain voltage threshold must be met. This can be achieved by comparing the voltage spike with a reference voltage that is unachievable by any excitation other than a radiation event. If the voltage spike surpasses this threshold, the comparator will output a digital "high" value, representing one event, and subsequently return to a digital low in preparation for the next event. These events can be summed with an FPGA digitally implemented counter, which will notify users of the total ionizing dose, as well as the dose rate, which is derived through the rate of change of the event count. Because there is no comparator circuit on the Triad chip, the output of an op amp will need to be buffered so as to replicate a comparator and produce a full-swing digital output that can be counted.

\subsubsection{Voltage Regulator and Frequency Synthesizer}

Two other components will be present on the radiation-hardened printed circuit board: the Microsemi voltage regulator and the FMI frequency synthesizer. The rad-hard voltage regulator, which is radiation hardened beyond 1 Mrad TID, will supply the voltage and current necessary for all circuits to operate. It will be powered by an external battery. The board was designed to be used with battery packs from $9 \mathrm{~V}$ to $12 \mathrm{~V}$, which encompasses the chemistries of $\mathrm{NiCd}, \mathrm{NiMH}$, lithium ion, and lead-acid. NiMH batteries 
have been ordered for this application since they are easily chargeable and readily available. Multiple copies of the voltage regulator will be present for circuits with varying voltage requirements. The radhard frequency synthesizer is capable of precise operation to at least $300 \mathrm{krad}$ TID and will serve as the clock generator for the FPGA controller. It is mounted on a so-called interposer board, which allows quick changes of the FMI ASIC.

\subsubsection{ASIC on the RHB}

Some additional components are necessary to provide the power required by the system and enable full circuit operation. All circuits within the system will need specific levels of voltage and current supply generated from a voltage regulator, and for radiation-rich environments, this supply must be very robust across a large range of dose rates, potentially up to $200 \mathrm{krad} / \mathrm{hr}$. Microsemi Corporation produces a variety of radiation-hardened voltage regulators with differing total dose capabilities, but the SGR117A model stood out with the highest TID capability of all, claiming total doses exceeding $1 \mathrm{Mrad}$. This voltage regulator can produce output voltages ranging from 1.25 to $34 \mathrm{~V}$, supply at least $1.5 \mathrm{~A}$ of current across all operating conditions, and maintain a $0.3 \%$ load regulation specification. The SGR117A is available in a 3-pin $\mathrm{K}$ package with a thermal dependence of only $3^{\circ} \mathrm{C} / \mathrm{W}$ and operating temperatures up to $150^{\circ} \mathrm{C}$. These voltage regulator specifications can satisfy power requirements for every circuit within the system, with the exception of the G-M tube, which will require a high-voltage DC converter to reach a $500 \mathrm{~V}$ potential. A photograph of the RHB is shown in Fig. 16.

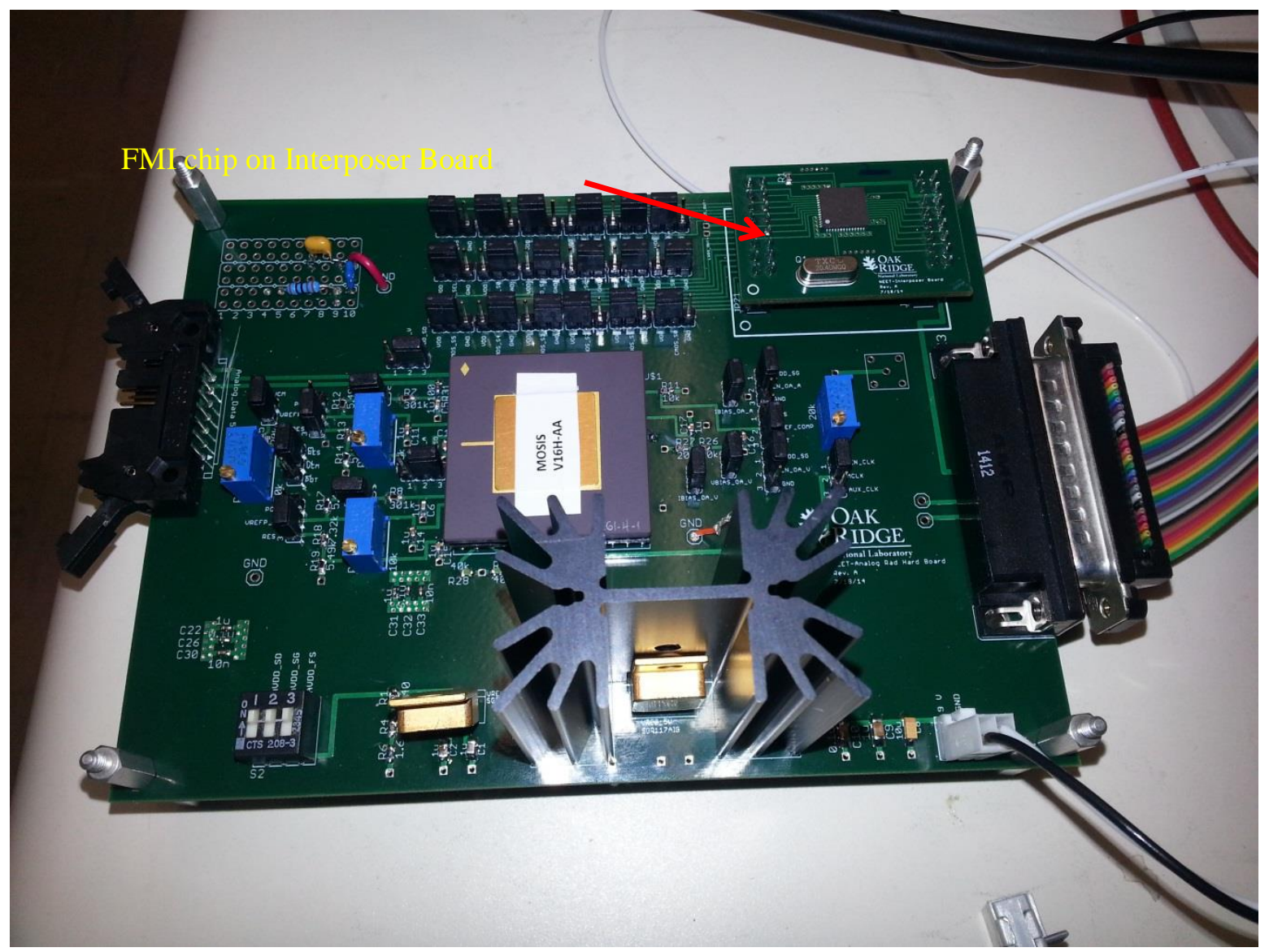

Fig. 16. The completed rad-hard board. 


\subsection{SENSOR BOARD}

As previously mentioned, the sensor board provides temperature, pressure, and gamma radiation detection elements. The analog device AD592 temperature sensor outputs a nominal current at room temperature $\left(\sim 25^{\circ} \mathrm{C}\right)$ of $298.1 \mu \mathrm{A}$ and will vary at $1 \mu \mathrm{A} / \mathrm{K}$. The sensor also requires a supply voltage between 4 and $33 \mathrm{~V}$, which will be provided by the selected Microsemi rad-hard voltage regulator. Placing a $10 \mathrm{k} \Omega$ resistor at the sensor output converts this current into a DC voltage of $2.981 \mathrm{~V}$ with a sensitivity of $10 \mathrm{mV} / \mathrm{K}$. This resistance value optimizes the $\mathrm{V}_{\text {out,DC }}$ and $\Delta \mathrm{V}$ components of the temperature-sensing circuit output voltage $\mathrm{V}_{\text {out }}$ so that the common voltage of the ADC is not exceeded and temperature change resolution is measurable. This voltage is input to rad-hard block sigma-delta modulator for digitization.

The Honeywell ASDX pressure sensor outputs a voltage that is dependent on gauge pressure and proportional to supply voltage. Based on the sensor characteristics, such as $5 \mathrm{~V}$ supply and 10-90\% calibration, the output voltage will be $0.5 \mathrm{~V}$ minimum plus $1.66 \mathrm{~V} / \mathrm{atm}$ above the sealed 1 atm reference pressure, up to $2 \mathrm{~atm}$ (Fig. 17). These voltage ranges are sufficient for analog-to-digital conversion without signal conditioning; therefore, this voltage is connected directly to the Triad sigma-delta modulator circuit input.

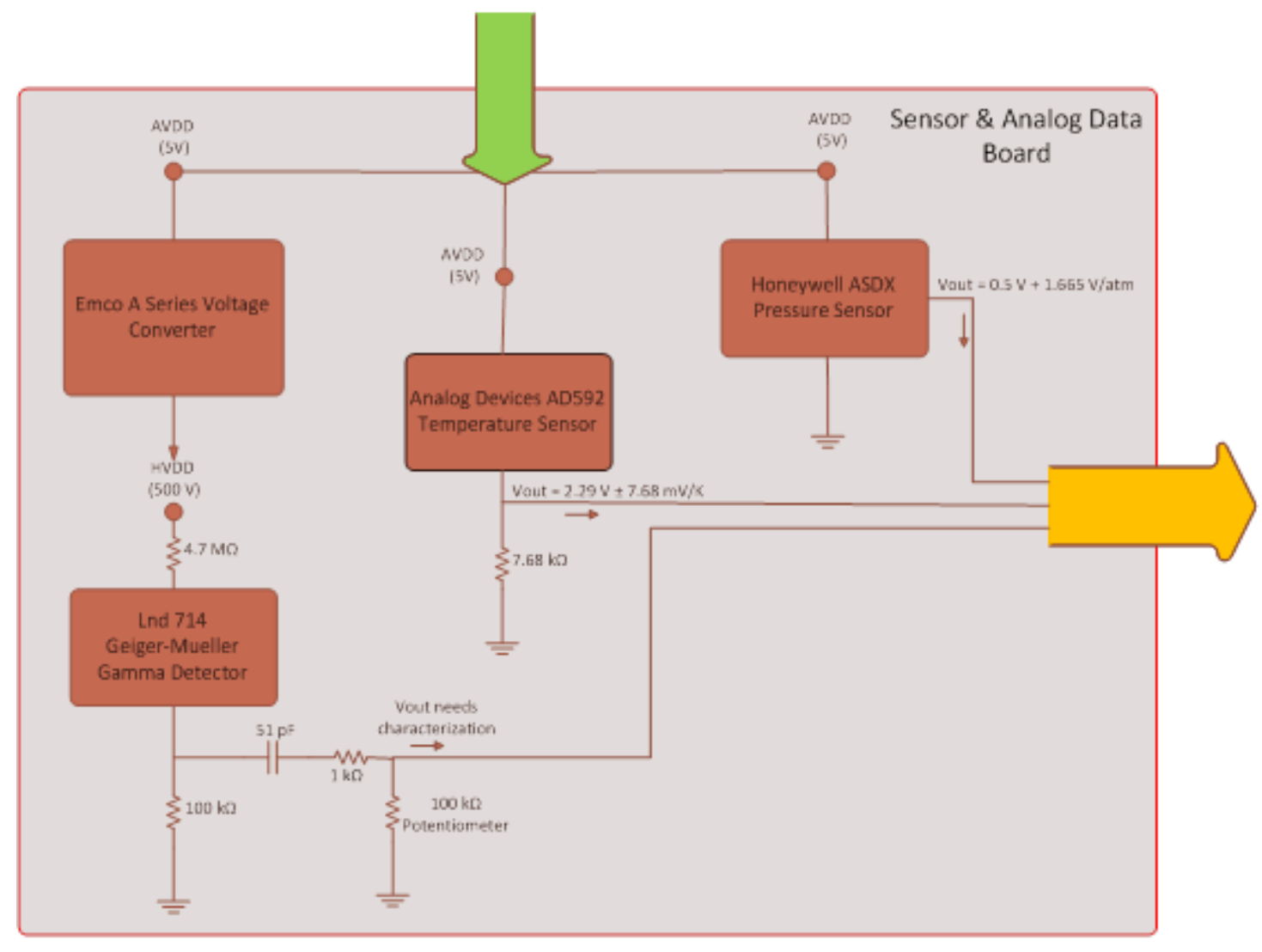

Fig. 17. Detailed sensor board block diagram.

The LND 714 G-M tube is a purely gamma radiation detection device; it requires a large electric field, in the range of $500 \mathrm{~V}$, in order to gather quickly moving electron-hole pairs freed by high-energy incident radiation. When a radiation event occurs, the G-M tube essentially "shorts" as a result of numerous charge carriers traveling toward their respective electrodes. Sizeable current-limiting resistors are required to prevent any significant current spikes as a consequence of shorting a $500 \mathrm{~V}$ electric field. As the G-M 
tube shorts, electrons move toward the positive supply, producing a fast positive current spike at the cathode. Since this waveform has a very high-frequency AC characteristic, it will take the low impedance path through the capacitor and be converted to voltage across the potentiometer. This voltage is then input to the rad-hard comparator for conversion to a digital logic signal. We have set up the GM-tube voltage and comparator thresholds using a $5 \mu \mathrm{C}{ }^{137} \mathrm{Cs}$ source.

The manufacturer of the LND 714 G-M tube recommends a $500 \mathrm{~V}$ potential for operation in order to capture high-speed freed electrons and holes, but a significant current supply is not required because the charge produced comes directly from the tube itself. The EMCO A05P5 $1 \mathrm{~W}$, positive adjustable DC converter can output up to $500 \mathrm{~V}$ at a maximum current of $2 \mathrm{~mA}$, with only a $5 \mathrm{~V}$ and less than $200 \mathrm{~mA}$ input at no load. This EMCO DC converter is not radiation hardened and will be placed on the shielded sensors board to minimize high-power transmission lengths and optimize board density. The $5 \mathrm{~V}, 200 \mathrm{~mA}$ converter input will be supplied by the Microsemi voltage regulator from the RHB. A heat sink will be added to the $5 \mathrm{~V}$ regulator to allow operation from both 9.6 V NiMH batteries and lithium ion $11.1 \mathrm{~V}$ devices. A photograph of the finished sensor board is shown in Fig. 18. The black coating is a highvoltage insulation material.

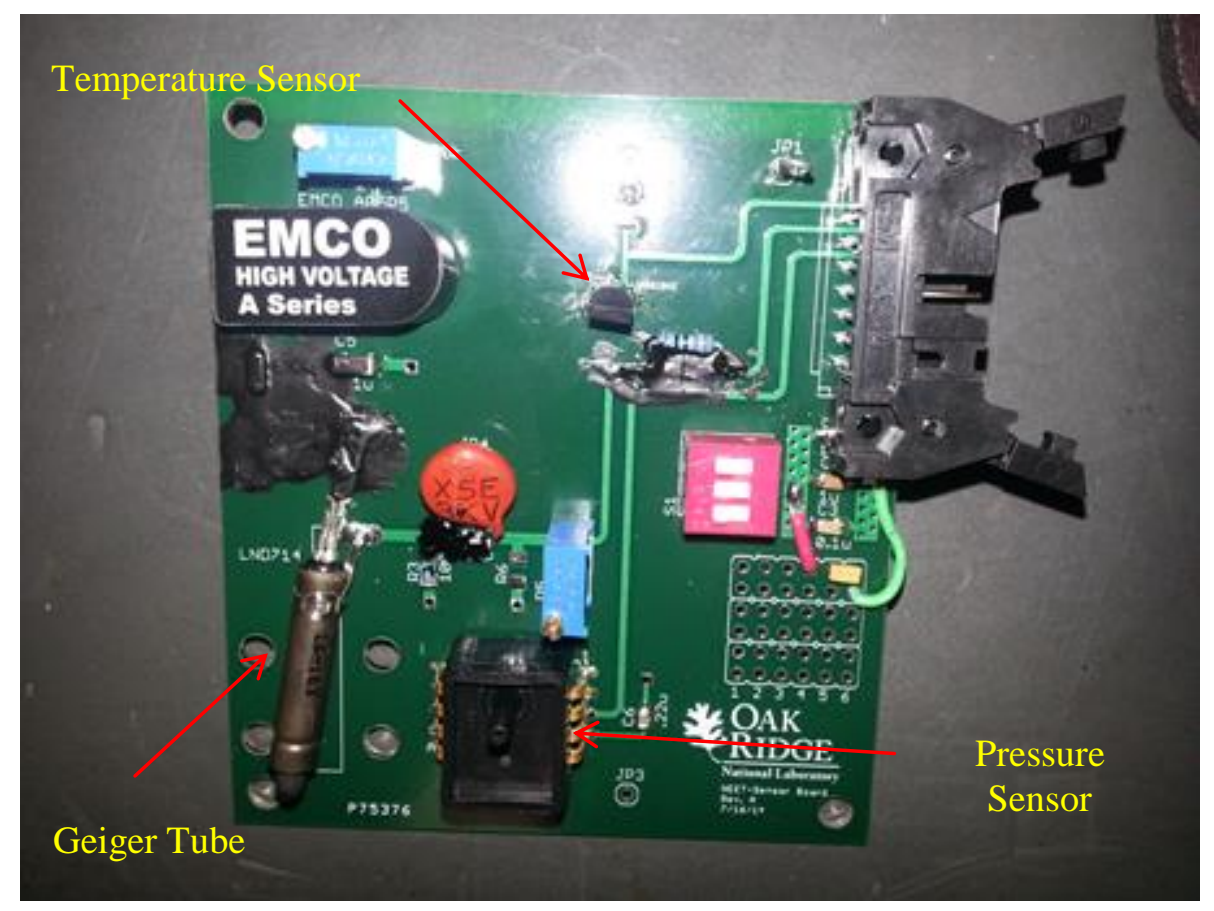

Fig. 18. The sensor board with insulation coatings.

\subsection{INTERFACE BOARD}

The function of the IB, shown in Fig. 19, is to translate the $5 \mathrm{~V}$ signals generated by the RHB and buffer and convert them to $3.3 \mathrm{~V}$ signals for the Nexys 3 board. In addition, the IB is used to perform the physical connections between the Nexys 3 board and the cable which connects the IB to the RHB and provides an ADC sample-clock/clock loopback from the Nexys 3 board if needed. The isolators are from the Texas Instruments ISO isolator family of interface devices. These circuits allow different supply voltages (in this case, $5 \mathrm{~V}$ and $3.3 \mathrm{~V}$ ) to be used on either side of the devices; they can transmit or receive digital pulses of the appropriate amplitude. 


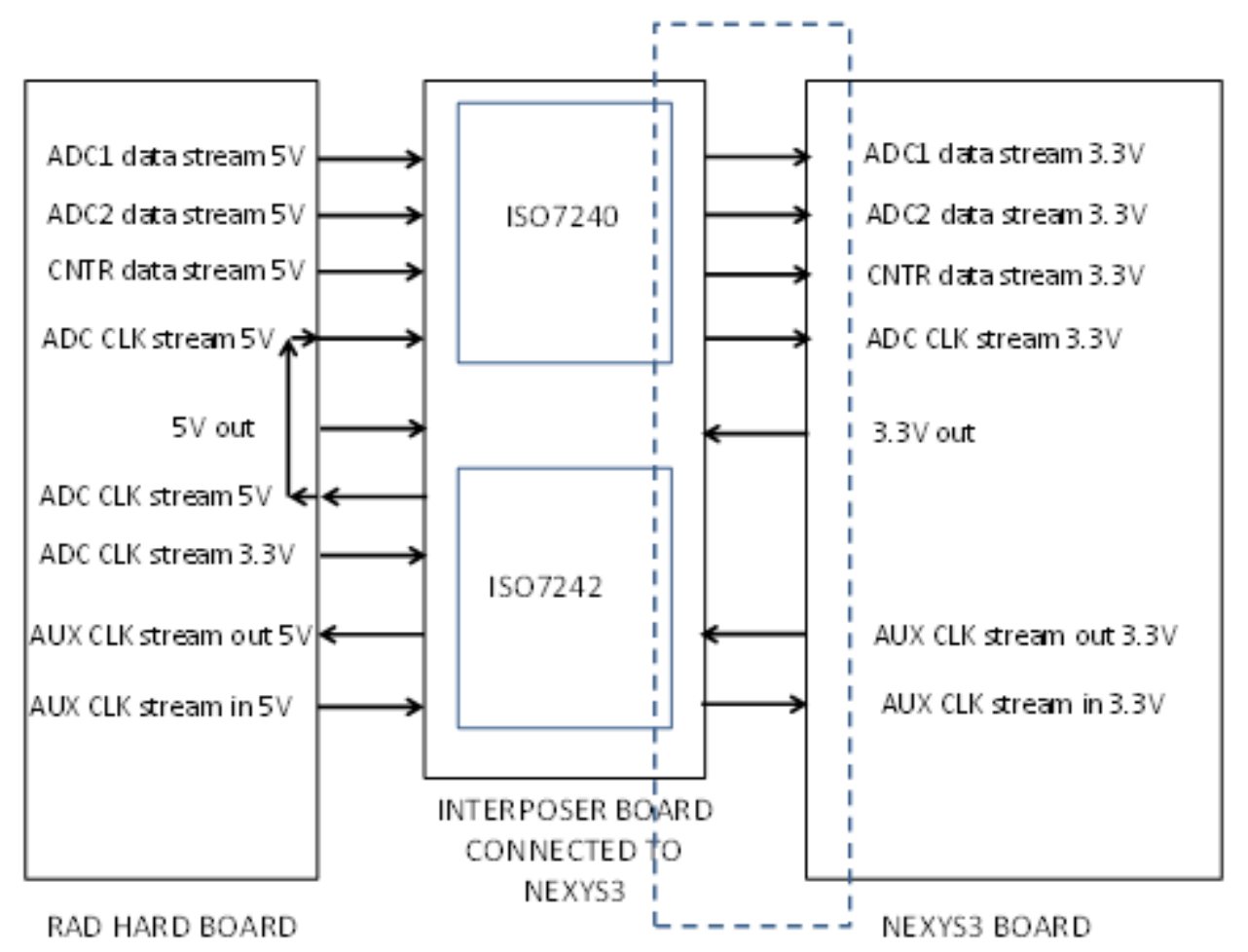

Fig. 19. Detailed interface board data flow block diagram.

\subsection{NEXYS 3 BOARD FIRMWARE/COMPUTER INTERFACE}

The program created for the FPGA controller was written as a state machine implementation of a universal asynchronous receive transmitter (UART) communications interface. The UART provides a serial data interface to a standard USB found on all modern computers. The USB interface provides fast data transfer between the data-collection computer and the rad-hard system. The electrical hardware portion of the interface, made by FTD International, is already resident on the Nexys 3 board (shown in Fig. 20) and requires code to implement the software functionality. The UART interfaces to a standard personal computer USB port and is configured to operate at 9600 baud.

Fig. 20. The interface board (right) connected to the Nexys 3 board.

UART (Block t serial) is the UART state machine code. It was taken from open-source VHDL code developed by Bainville [5] and modified for this project's purpose. The code implements a single-byte receive and retransmit UART which was minimally modified to receive a command byte and then retransmit either a status byte or a data byte, depending on the code sent. The UART is programmed to receive any of $\mathrm{X}$ different ASCII bytes and then parse them in a state machine. If the byte is a valid command, there is a predefined action that takes place. A list of the commands is as follows.

COMMAND

hex 20 (space)

hex $21(!)$

hex $22(")$
FUNCTION

system-wide reset

counter reset

system-wide data load
RETURN CHARACTER

hex 20 (space)

hex 21 (!)

hex 22 (") 
hex $30(0)$

hex 31 (1)

hex 32 (2)

hex 33 (3)

hex 34 (4)

hex 35 (5) counter high-byte load

counter low-byte load adc1(temp) low-byte load adc1(temp) high-byte load adc2(press) low-byte load adc2(press) high-byte load (data)

(data)

(data)

(data)

(data)

(data)

For these commands, "temp" means the temperature ADC and "press" means the pressure ADC. The command architecture is designed such that combinations of these commands can be issued to perform a greater overall function than any single command. For example, issuing system-wide reset, system-wide data load, adc1(temp) low-byte load, adc1(temp) high-byte load would read the entire data from the temperature ADC for a single reading.

Counter (Ctr 16 dp) maintains a running count of the individual events received from the Geiger-tube comparator. After an interval of time determined by the computer software, the counter value is transferred 8 bits at a time ( 2 bytes) to the computer. Each data transfer requires a transfer command. The counter is then reset and allowed to begin counting again.

Decimation Filter (Decimation_filter_top) filters out the out-of-band digitization noise from the sigmadelta modulator data. Input to the filter is a single-bit data with variable pulse density that corresponds to the signal level at the modulator/ADC input. In this particular case, the input data represent slowly changing temperature and pressure measurements. For all practical purposes, the modulator input is considered a DC signal.

The decimation filter is implemented as a third-order Cascade, Integrate and Comb (CIC) low-pass filter (Fig. 21). The "N" represents the filter order, and the " $\mathrm{R}$ " is the filter decimation ratio. In this application, $\mathrm{N}=3$, and $\mathrm{R}=256$.

\section{Integrator Section Comb Section}

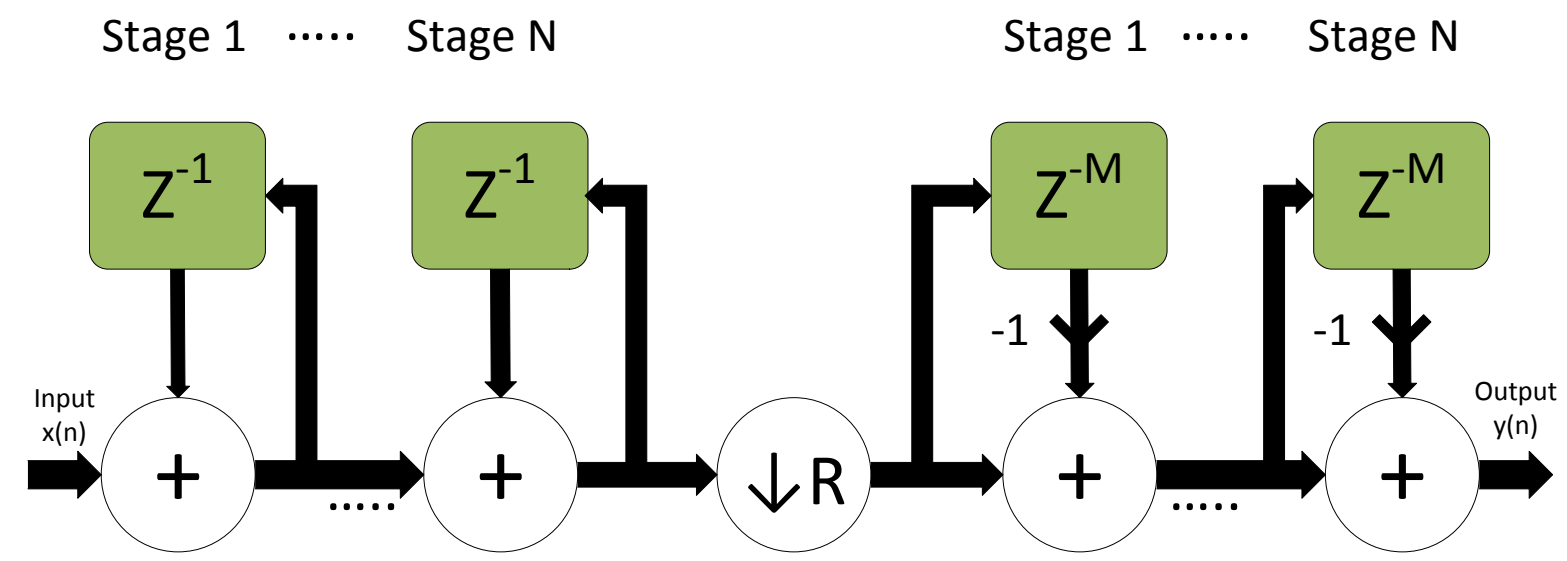

Fig. 21. Decimation filter mathematical structure. The "N" represents the filter order, and the " $R$ " is the filter decimation ratio. In this application $\mathrm{N}=3$, and $\mathrm{R}=256$.

The size of the filter is $1 \mathrm{bit}$, and the output size is 16 bits. The filter's magnitude frequency response without the decimation is shown in Fig. 22. 


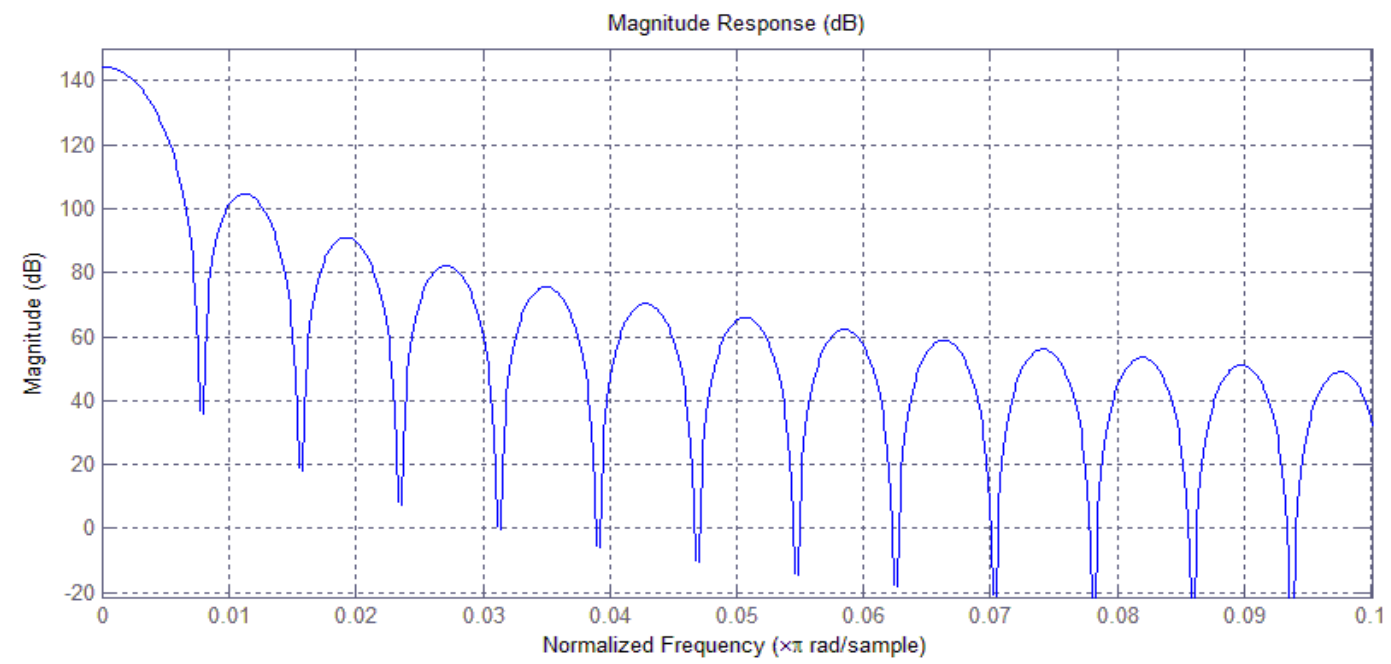

Fig. 22. Decimation filter frequency response.

The performance of the decimation filter was simulated using Simulink. When using the first-order modulator to feed the decimation filter, the dynamic range of the filter's output was around 80, which corresponded to more than 13 bits of resolution. The decimation filter structure from Fig. 21 was implemented in FPGA, and its proper behavior was confirmed in hardware testing.

Clock Generator (ClkGen) is a backup clock generator implemented as a frequency divider off of the $100 \mathrm{MHz}$ Nexys 3 system clock. The output value of the clock is $100 \mathrm{MHz} / 2^{6}$ or $1.5625 \mathrm{MHz}$.

\subsection{LABVIEW INTERFACE PROGRAM}

The LabView interface program collects data from the Nexys 3 board programming and displays the value of the data. It transmits the selected command outlined in the UART section and displays the return value of the requested command. It also configures the COM port on the computer to the appropriate settings. A screen shot of the program is shown in Fig. 23. 


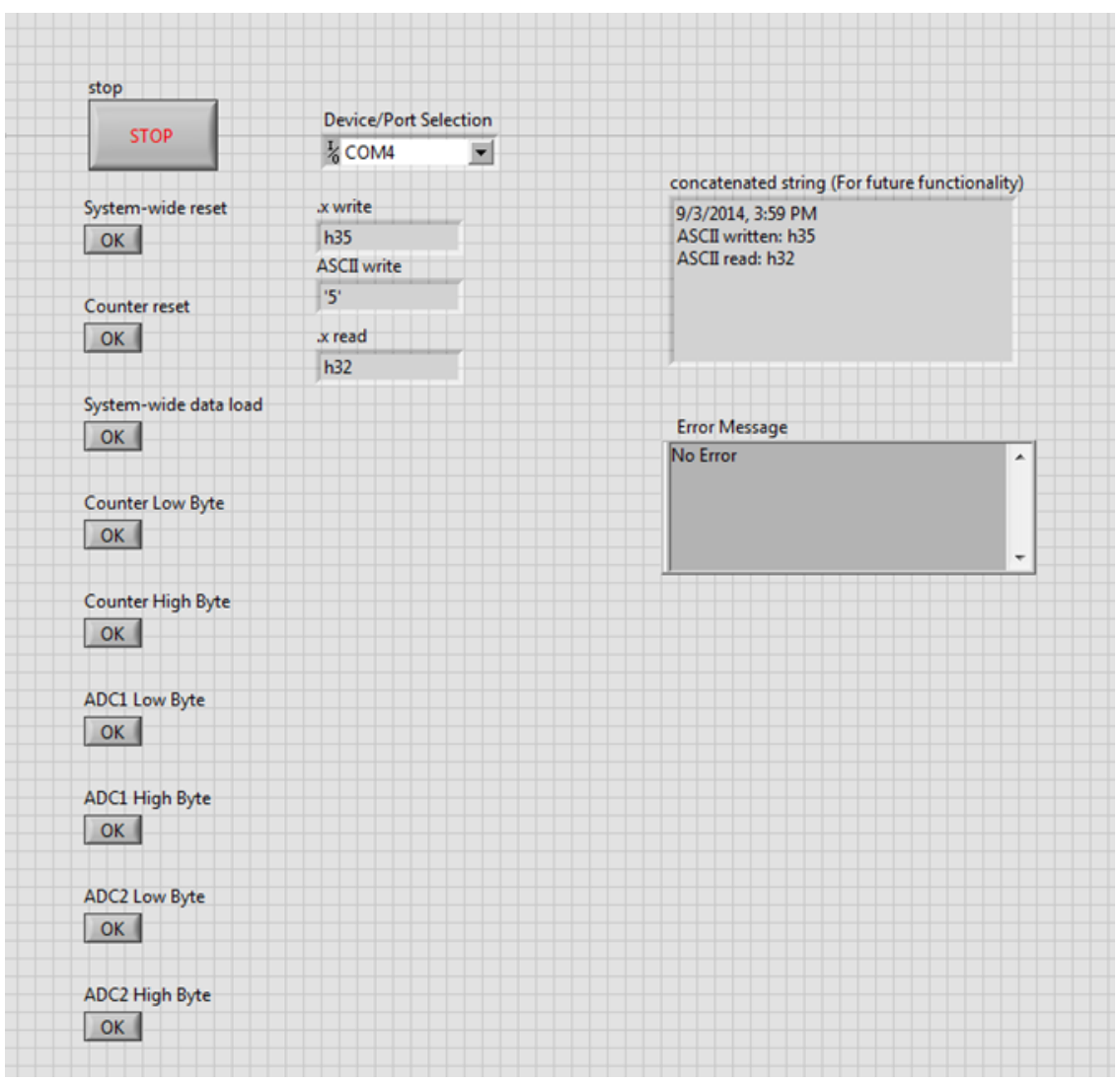

Fig. 23. Screen shot of the LabView interface program.

\subsection{RADIATION TESTING SETUP}

\subsubsection{Site Selection}

There are many parameters that categorize a radiation test site, including radiation source type, dose distribution, and dose rate. All of these parameters can vary greatly, depending on the exposure facility. The application often determines which configuration would be best for obtaining realistic, useful system radiation-tolerance data. For the data acquisition system in particular, we are interested in TID radiation potentially up to $300 \mathrm{krad}$ at a target dose rate of $30 \mathrm{krad} / \mathrm{hr}$. In addition, a $360^{\circ}$ dose distribution also is useful for this system due to the large area required (approximately $4.5 \mathrm{in} . \times 6$ in.) of the RHB to mount all of the necessary ASICs. It will help provide an even distribution of radiation dose across the entire board area and will ensure proper radiation exposure to all circuit elements designed for radiation hardness - as would be expected during an immersive exposure to high-energy emissions characteristic of reactor accident scenarios.

TID radiation in the environments of interest comes primarily from gamma rays or high-energy photons. The most common and cost-efficient radiation source that fits these specifications by nuclear decay (a much more convenient photon source than a high-energy X-ray generator) is cobalt-60, which has photopeaks at 1.186 and $1.348 \mathrm{MeV}$. Numerous radiation exposure facilities house cobalt-60 sources, but all specifications must be met. Many facilities were considered, including options on site at ORNL. The decision was made that a laboratory at Arizona State University, under the direction of Dr. Keith Holbert, was the best fit for our application. This lab is equipped with a Gammacell 220, which is a $360^{\circ}$ distributing cobalt-60 source. This cell provides an isotropic dose (Fig. 24) that produces a dose rate of $28.62 \mathrm{krad} / \mathrm{hr}$ during the time period of radiation testing. A chamber that contains the devices to be 
irradiated is lowered into the pool with a path out the chamber's roof for cabling. Dr. Holbert also is a primary investigator for another project under Nuclear Energy Enabling Technologies (NEET) 2 (project CT-14ID070402).

\section{ISODOSE CURVES Gammercoll 220}

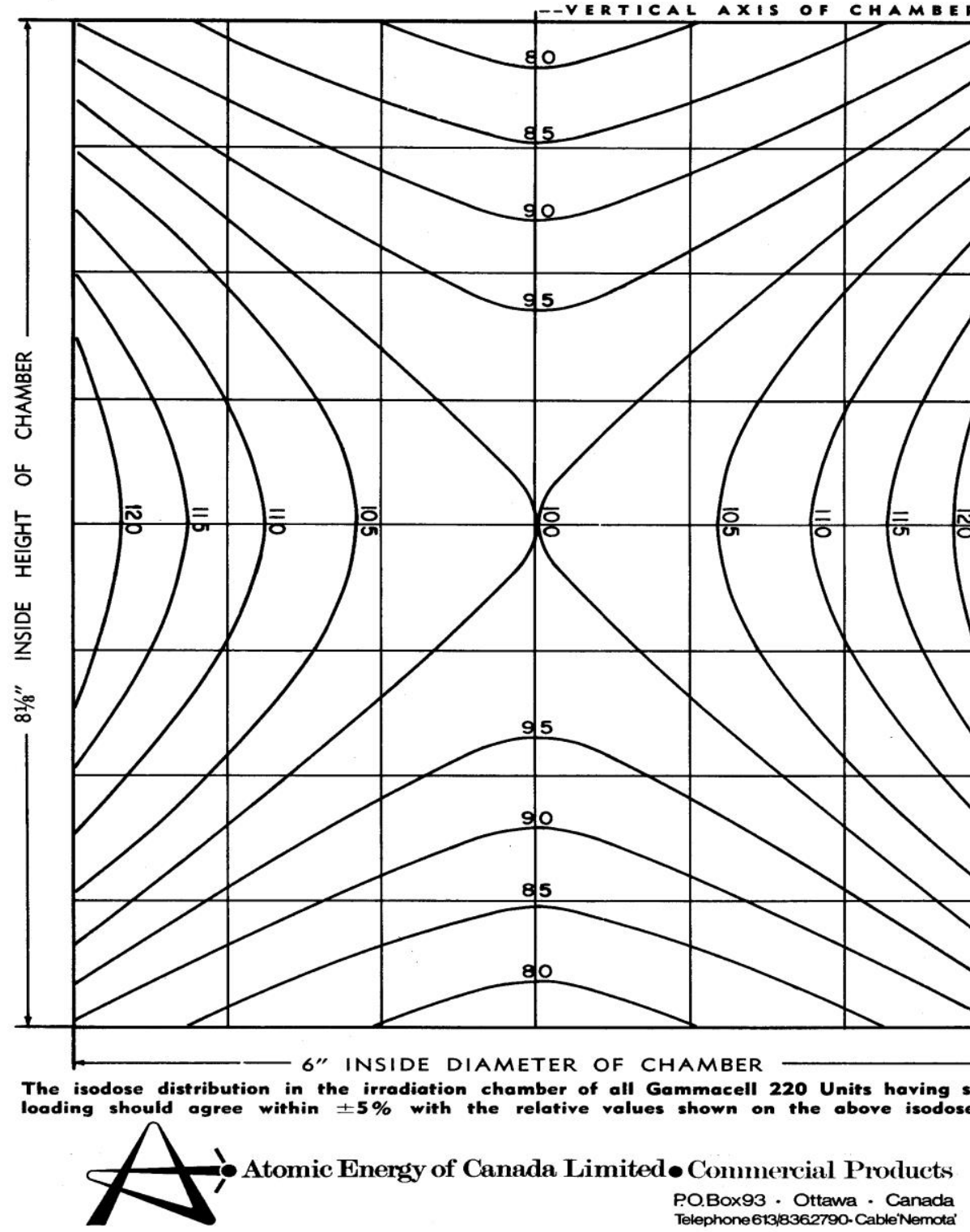

Fig. 24. Gammacell 220 isodose curves.

\subsubsection{Test Setup}

Radiation detection is the key parameter in identifying a nuclear reactor disaster scenario. Radiation can and will escape reactor containment before an event-related change to ambient temperature or pressure is measured. A test setup that exposes the proper boards to gamma radiation and protects the vulnerable 
components from harmful dose rates, all while allowing ambient parameter and power-consumption data to be taken, is crucial to the accuracy and prolonged operation of the entire system.

Because only the RHB will be lowered in the radiation pool at the test facility, multiple cables and wiring will need to be irradiated as well. This includes the power cable from the voltage supply, data and power cables to the sensor board, and data and power cables to the FPGA controller board. These cables will need to be recycled after each system is irradiated because the polyvinyl chloride insulating material will begin to deteriorate at high TID. A final rad-hard system used on a robot would be constructed with shielded cables or with mineral-insulated cables. Also, since the G-M tube will be in use to monitor any background radiation, the sensor board will need to be placed in a high-voltage protection box to safeguard against any incidental contact with the existing voltage. In addition, power consumption is an important parameter that is susceptible to radiation effects if not properly protected or otherwise hardened, so system voltage and current are measured for the duration of the test. This is done by simply connecting a voltmeter across the terminals of the voltage supply as well as an ammeter in series with the positive referenced current supply. Because of the immobility of direct-current power supplies, a battery better suits the application of rad-hard data acquisition for nuclear reactor monitoring compared to a wired system; thus, a $9.6 \mathrm{~V}$ NiMH battery is used as the power supply during radiation testing. Figure 25 illustrates the radiation testing setup.

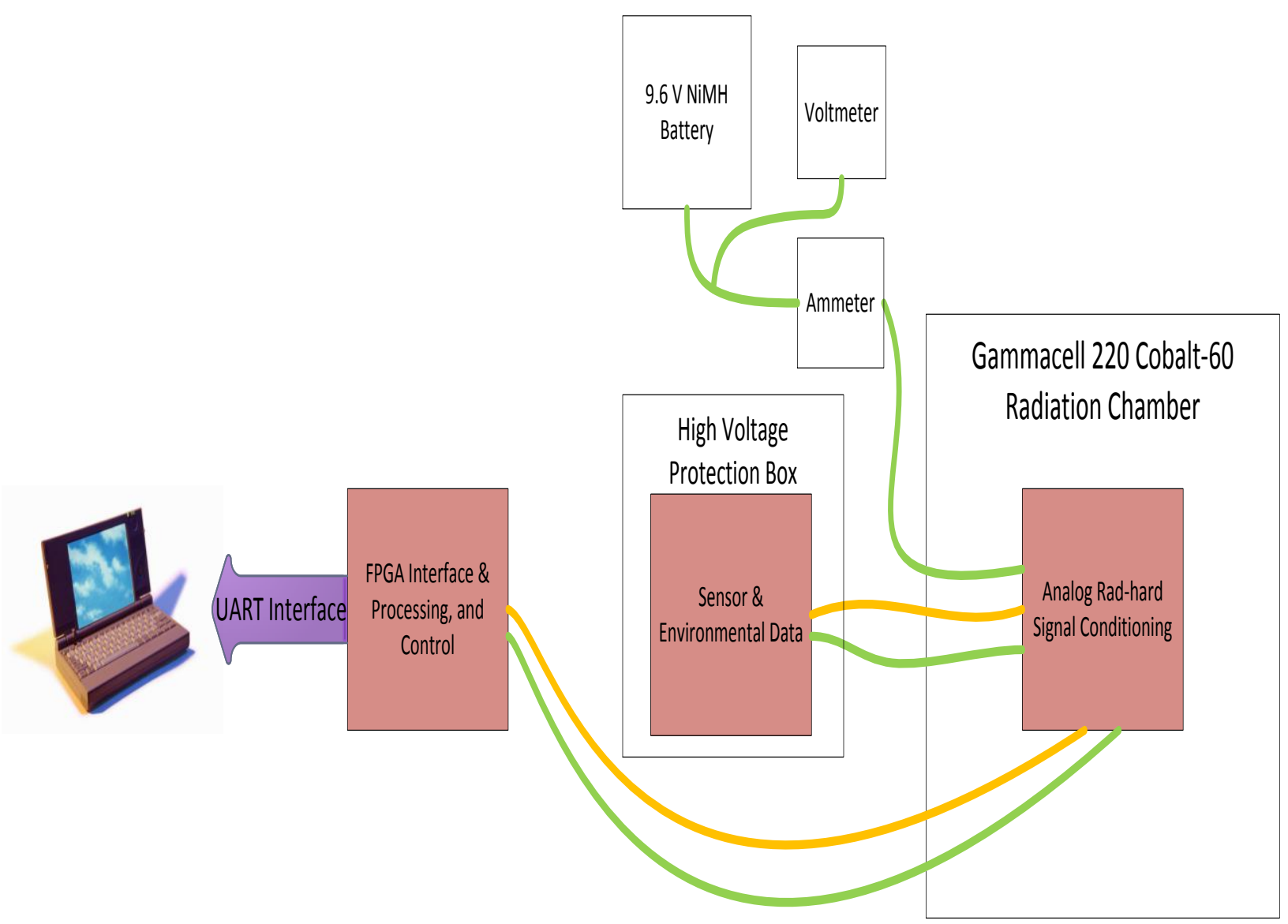

Fig. 25. System radiation testing setup. 


\subsection{LABVIEW INTERFACE SOFTWARE CODE DEVELOPMENT}

The previous version of the LabVIEW interface program collected data from the Nexys 3 board programming and simply displayed the value of the data. It transmitted the selected UART command and displayed the return value of the requested command. It also configured the COM port on the computer to the appropriate settings. That version was sufficient to gather data during calibration testing but was unable to use the curve coefficients found during calibration to convert the raw digital data back to useful ambient data. The final goal of the data acquisition system is to provide intelligible data to the end users so that they can assess a reactor environment operating condition. Modifications to the LabVIEW software were necessary to allow temperature and pressure to be directly displayed. Mathematical functions such as multiplication, division, addition, subtraction, square roots, and squaring were implemented to enable solving of equations. These equations differed between the temperature and pressure calibration coefficients because the temperature curves use a polynomial fit and pressure curves use a linear fit. In addition, because five complete systems were calibrated for both temperature and pressure, an array subset function was implemented to allow users to select which system is being tested and hence which set of calibration coefficients to use.

The converted data also needed to be actively plotted so users can visually discern the operating conditions of the system's ambient environment as a function of time. This required using LabVIEW's graphing palette and array builder to construct an array of $x$ and $y$ coordinate points, $x$ being time and $y$ being ambient data. This was done for all three sets of ambient data: temperature, pressure, and gamma radiation events. The temperature and pressure displays need be instantaneous, so a simple plotting of the $x$ and $y$ coordinate points would suffice. The gamma event display, however, should be a dose rate measurement. This means that the $y$ data are normalized to number of counts per second. This conversion requires calculating the time elapsed between each data point and dividing the number of counts that occurred within that cycle by the measured time. Then a true dose rate with units of counts per second can be used to create an array that can be plotted by LabVIEW's graphing block. All of these graphs, along with all other functionality implanted within the LabVIEW software used for final testing, are shown in Fig. 26.

Third, all of the data in the arrays used for visual graphing not only need to be displayed continuously to the end user for active monitoring but also should be stored as pure data into a local file for later examination. For each system tested, a separate location can be assigned to write the data into a specific comma-separated value (csv) file as the arrays are updated. LabVIEW features a block called "write to spreadsheet" that executes this functionality with options such as append file, transpose data, and data formatting for complete customization of the csv file in which the data are written. 


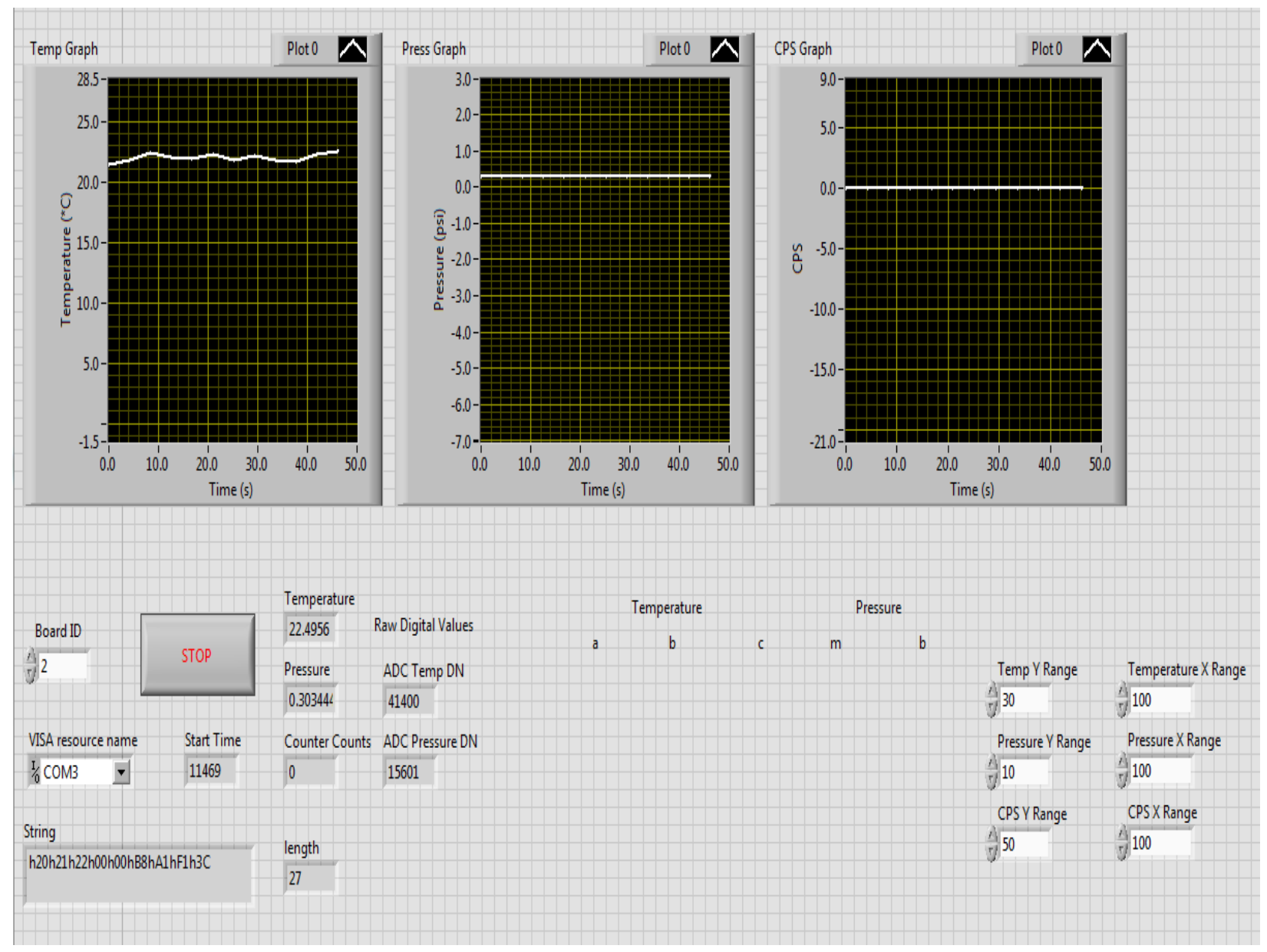

Fig. 26. LabVIEW interface software used for calibration and radiation testing.

\section{PREIRRADIATION CALIBRATION TESTING}

The rad-hard data acquisition system for Task III of the NEET 2 project was partitioned across three board designs because of the extremely high cost of rad-hard parts such as sensors and FPGAs. One board houses all the necessary rad-hard ICs, such as the Triad VCA, the FMI frequency synthesizer, and voltage regulators. Commercial sensors could be mounted on one of the remaining two boards, and a digital controller could be mounted on the other. Because the system is spread across three boards, the required cabling and longer wire traces create parasitic power and signal drains that induce some variations between individual systems. Inherently, there will be system-to-system variations regardless of board partitioning because the IC fabrication process, although precise, is not perfect, and silicon substrate variations from chip to chip are inevitable. Although these variations at the chip and board level typically are small, they need to be accounted for in order to preserve the integrity of the data acquisition process.

An easy way to mitigate variations is to calibrate each system individually. This includes recording the final system output DN of each data path for a specific known input value. The input value is swept across the entire range of possible inputs at a certain interval, and data are recorded at each step. If enough data points are taken, the final output DN then can be plotted vs the input range to portray each system's specific output value curve for the selected input range. Each curve is fitted with an equation, and thus output DNs can be mapped back to input ambient environmental data precisely for each system 
for easy display to the end user. Curve fitting is performed empirically by selecting the type of curve that best approximates the system's calibration data, which is quantified with a coefficient of determination, or r-squared, value. An r-squared value always falls between 0 and 1 . A higher value denotes a better fit of the selected curve type to the measured data than a lower one. After a curve type is selected, the coefficients of the equation describing that curve, or trendline, can be extracted and used to directly convert digital outputs to proper inputs. The coefficients are static for each system and only need to be measured once. LabVIEW then uses them to automatically calculate the ambient input data based on the instantaneous system output.

\subsection{TEMPERATURE CALIBRATION}

The main focal points when analyzing calibration data are linearity and continuity. Linearity is important to ensure that the ADC adds minimal distortion to the waveform being processed. Continuity is important to ensure that there are no inputs for which an output code is missing. The temperature ADC data in Fig. 27 show great linearity over the entire ADC input dynamic range at an r-squared value of 0.9998 .

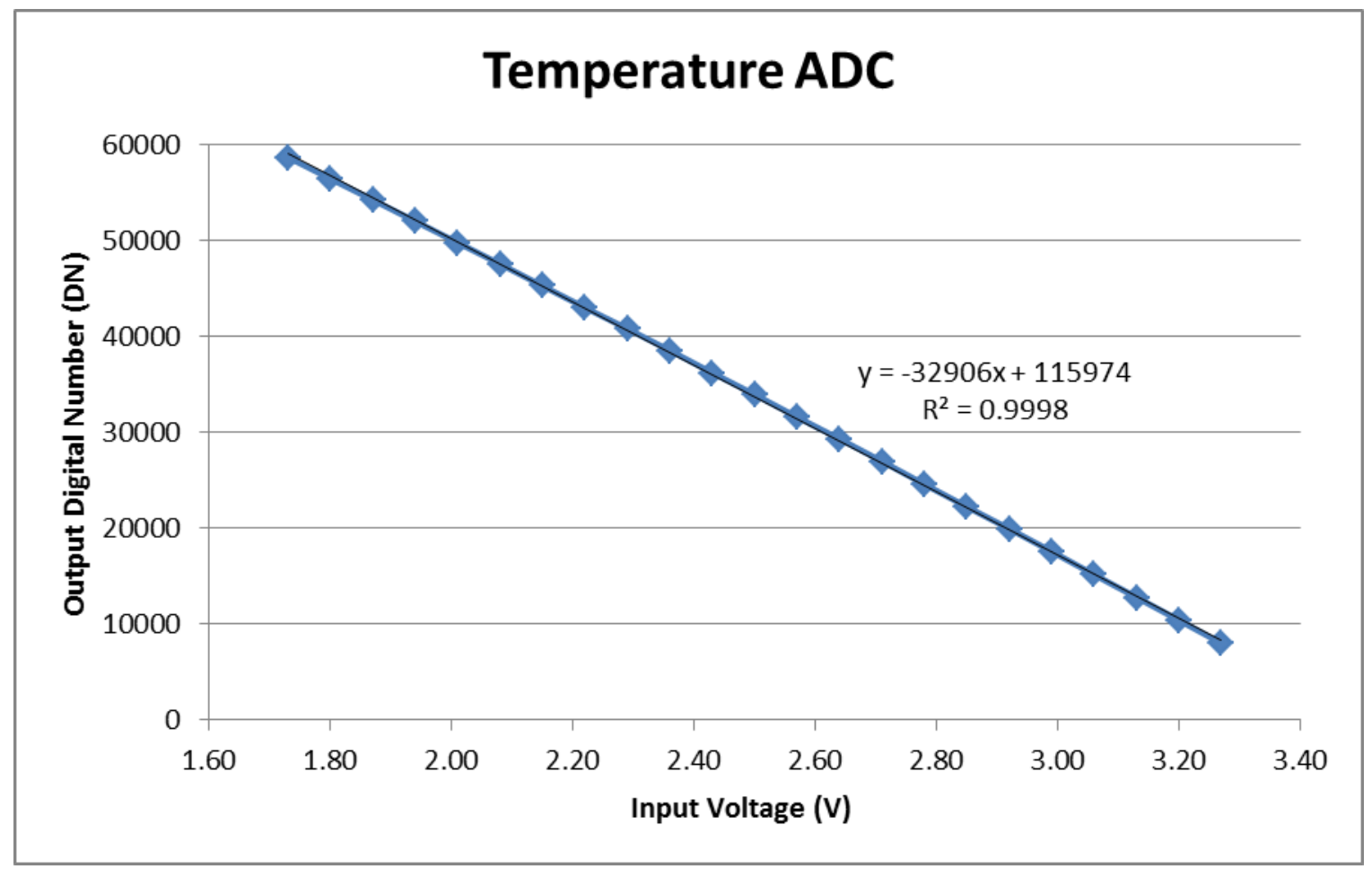

Fig. 27. Preliminary temperature ADC output DN vs input voltage.

Based on the excellent linearity of the circuits within the temperature data path on the RHB, it can be stated that any nonlinearities in the overall system calibration measurement are attributed to the sensor board components. Some slight exponential behavior is expected from the sensor board. That behavior is attributed to the requirement of a current-to-voltage resistor on the output of the temperature sensor. It is well known that resistance changes to some degree as a function of temperature, even with highprecision, low-temperature-coefficient resistors. The sensor board is subjected to the most extreme temperature range of any board in order to model realistic operating conditions. Thus there will be an inevitable shift in the current-to-voltage resistance, resulting in a voltage shift as well, according to Ohm's law. 
Our published final project specification [1] requires five complete data acquisition systems be functional and tested across temperature and pressure. The result of temperature calibration for all five systems is shown in Figs. 28 through 32. The blue data points represent sweeping the temperature of the environmental chamber from $-5^{\circ} \mathrm{C}$ to $70^{\circ} \mathrm{C}$ in $7.5^{\circ} \mathrm{C}$ increments with only the sensor board inside the chamber The RHB and controller board are left outside the chamber in ambient room temperature.

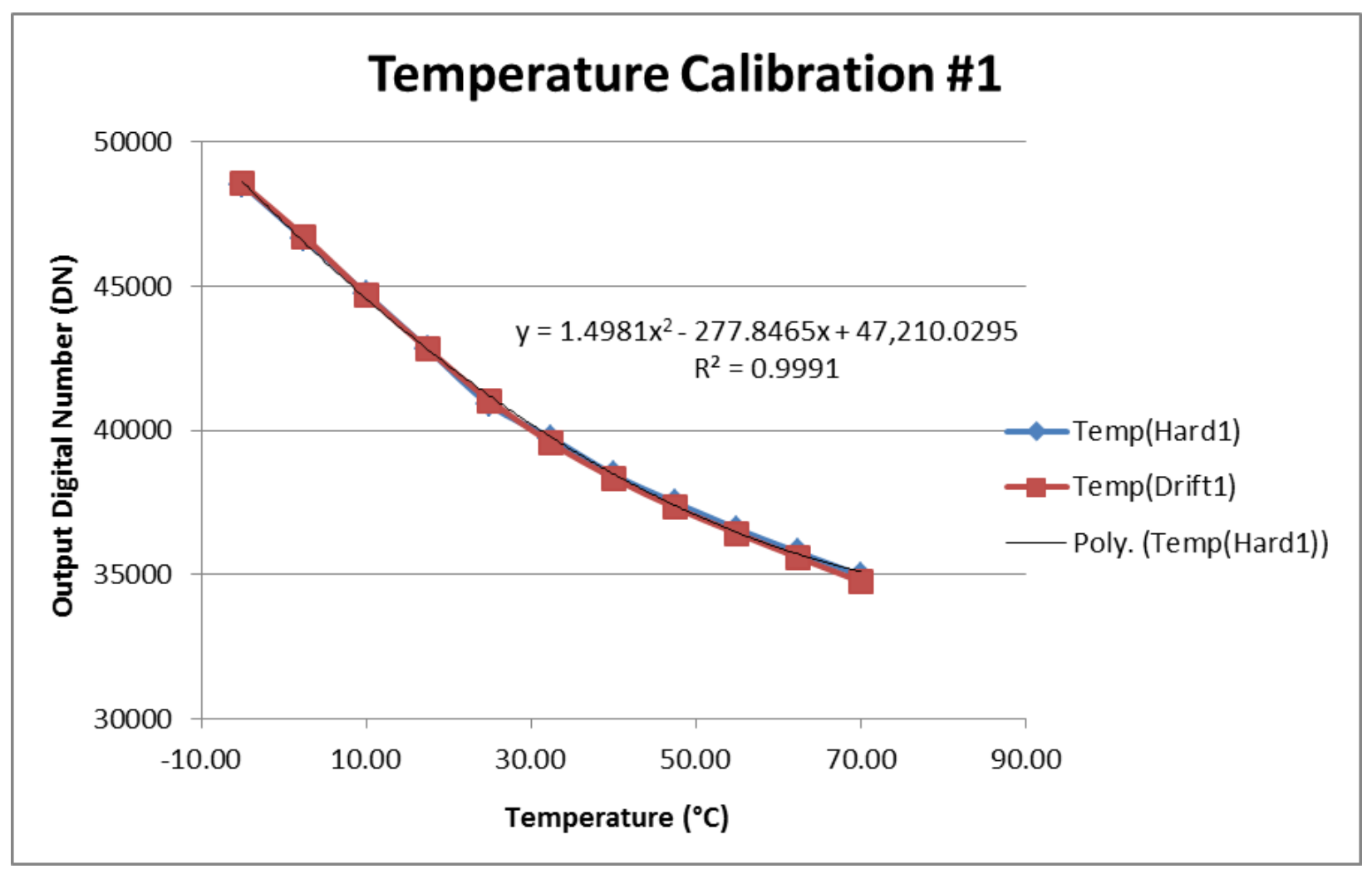

Fig. 28. System 1 temperature output DN vs ambient temperature. 


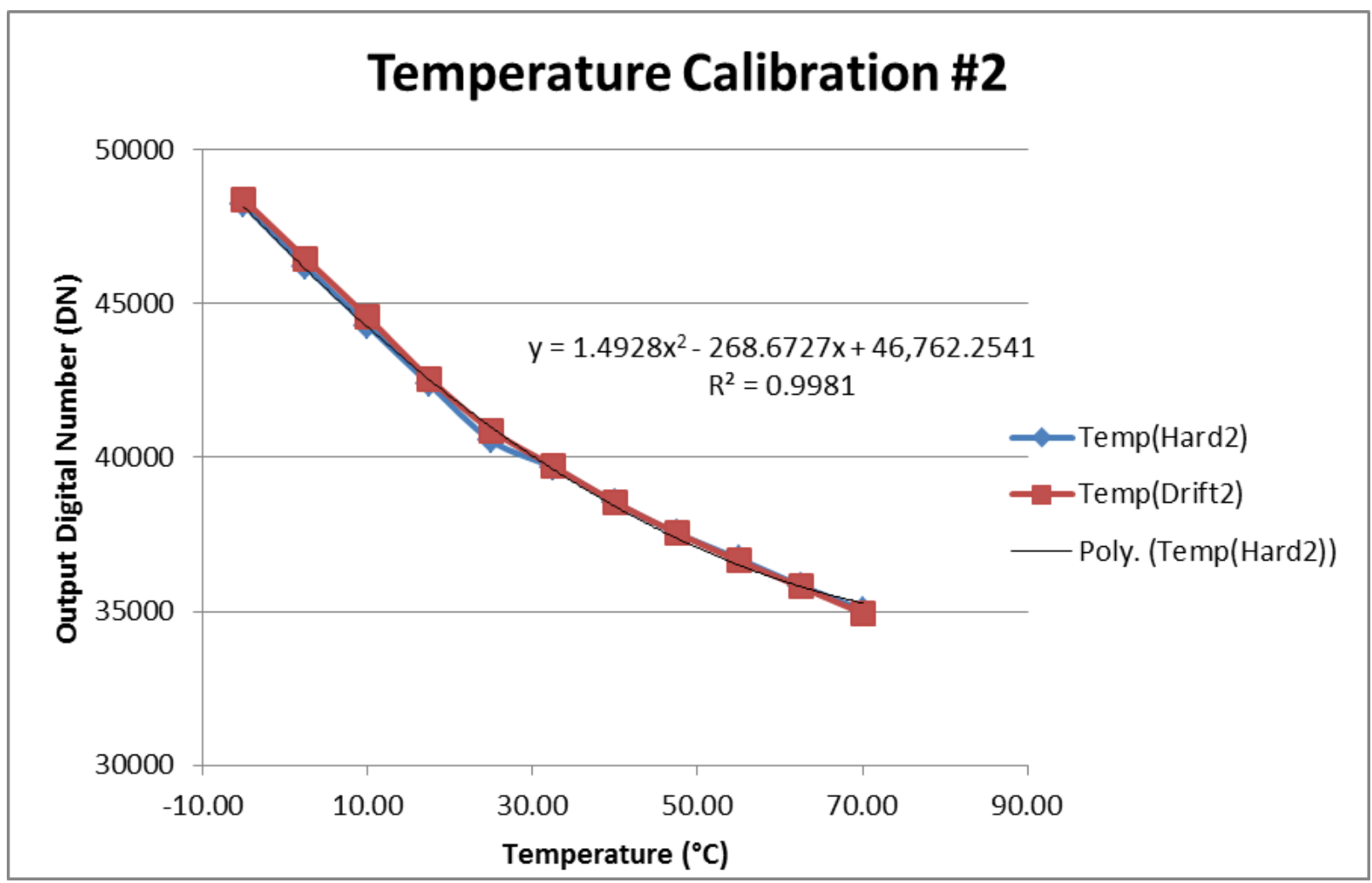

Fig. 29. System 2 temperature output DN vs ambient temperature.

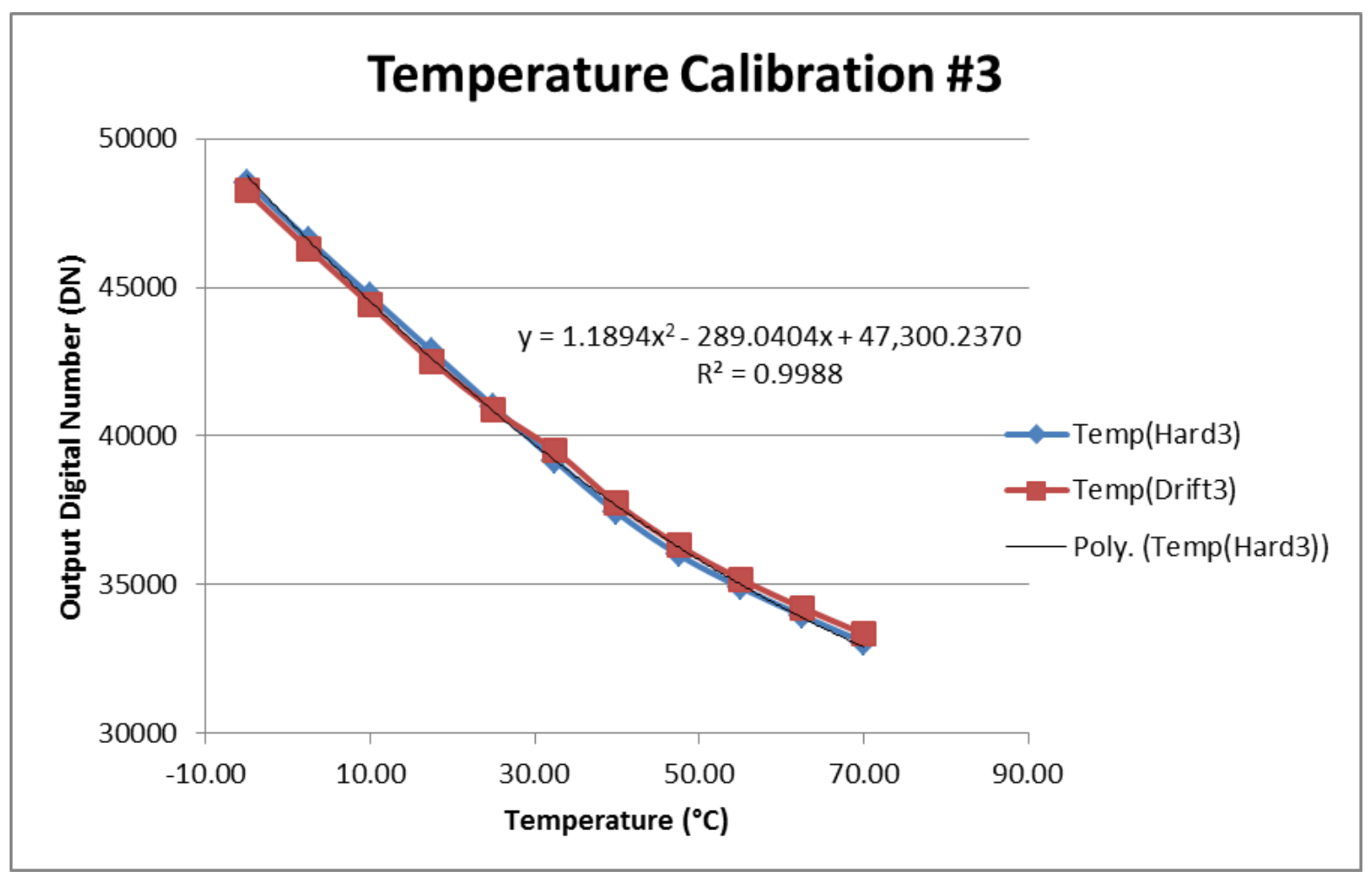

Fig. 30. System 3 temperature output DN vs ambient temperature. 


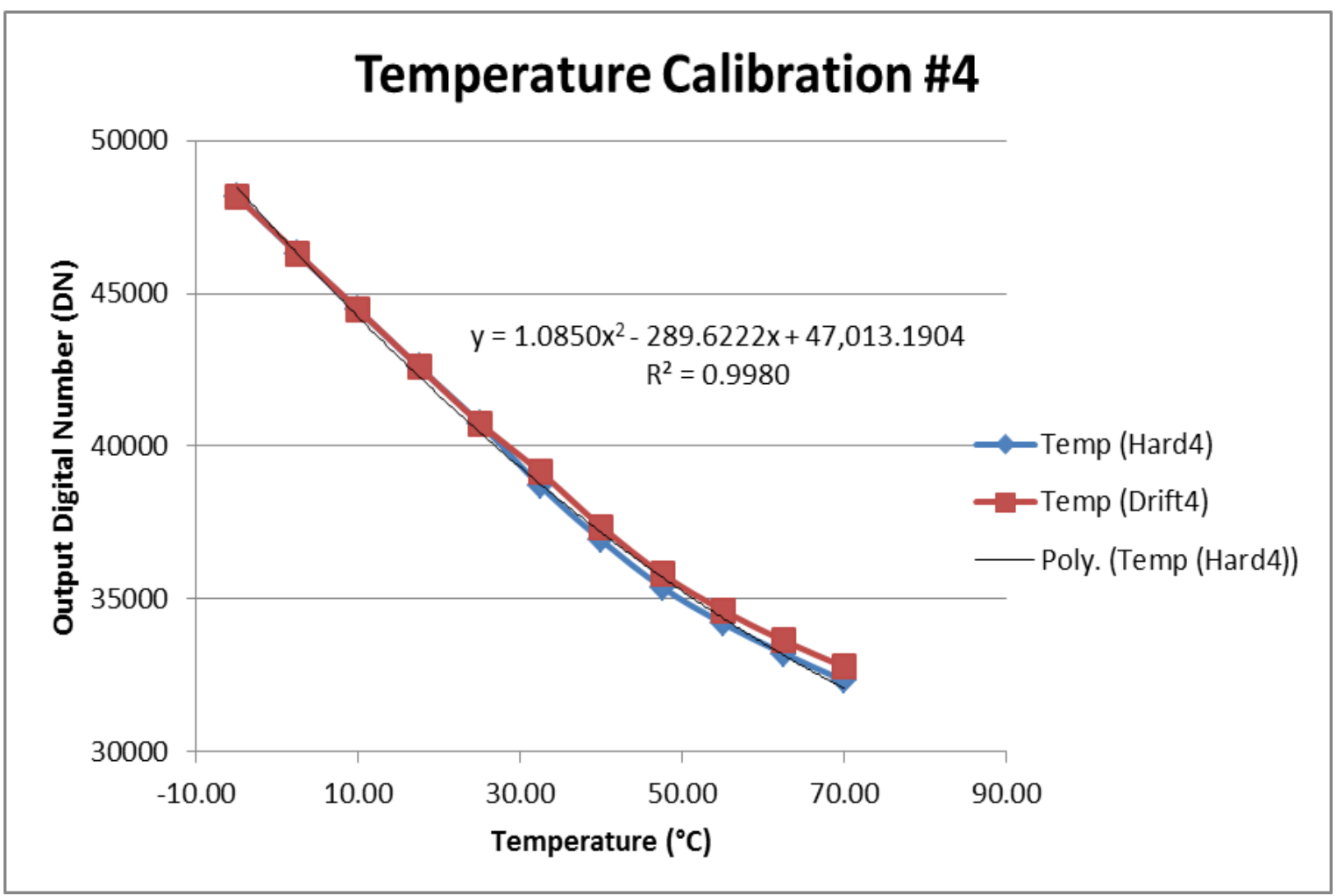

Fig. 31. System 4 temperature output DN vs ambient temperature.

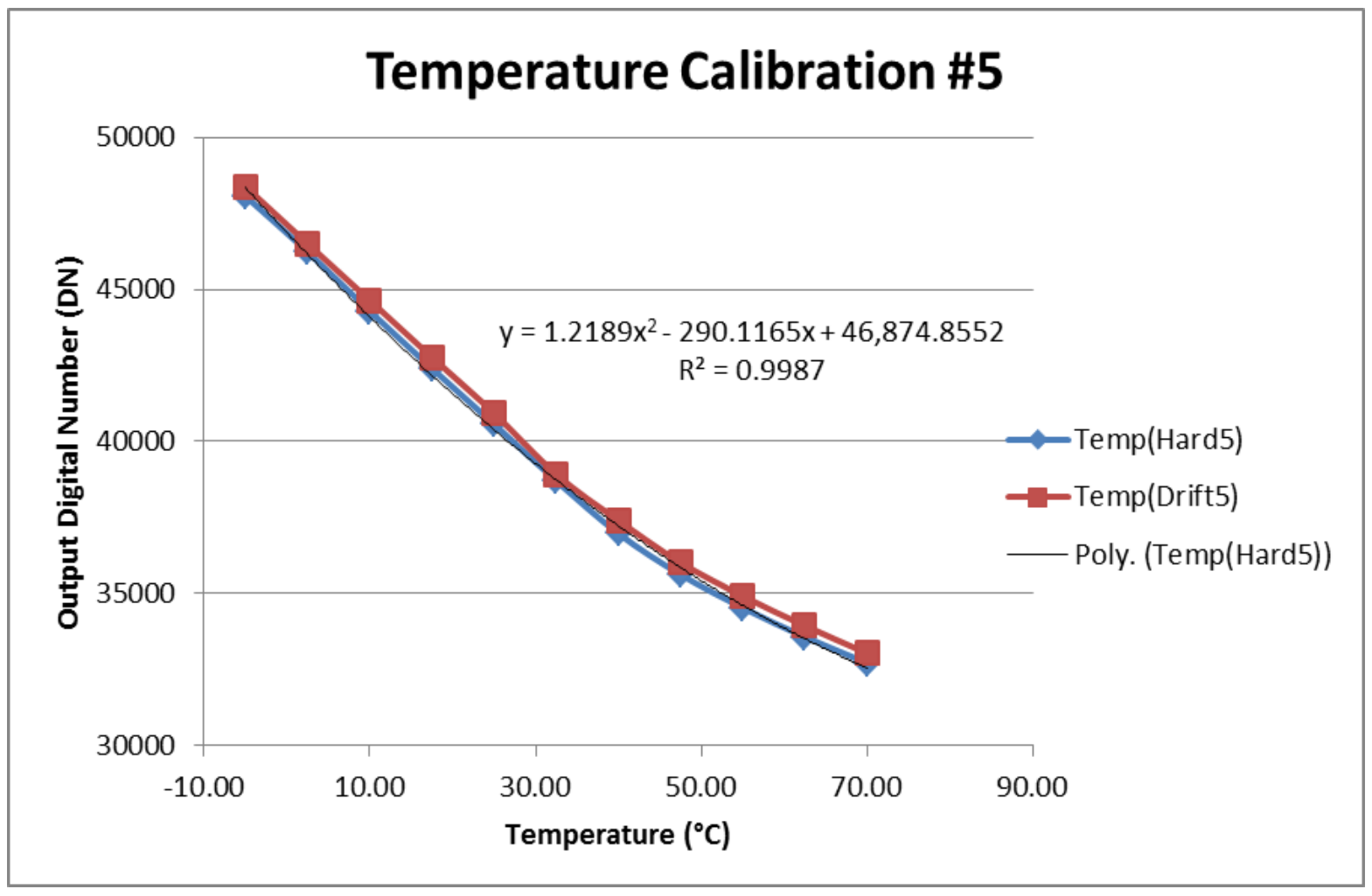

Fig. 32. System 5 temperature output DN vs ambient temperature. 
As shown in the temperature calibration data, there is some apparent nonlinearity with an upward concavity. This behavior was expected and is in line with a concept known as resistance shifting: As the chamber temperature decreases, the current-to-voltage resistance also decreases, resulting in a downward shift in voltage. This voltage reduction produces an increase in output DN. Conversely, the opposite shift happens when chamber temperature increases. This behavior results in a concave-up curve of plotted DN vs temperature data.

Given the slight nonlinearity of the temperature calibration data, a linear trendline will not best approximate the curve. All five other regression types were examined, and a second-order polynomial approximation produced the highest $r$-squared value for all five systems. Table 4 summarizes the polynomial r-squared values and coefficients that are used by LabVIEW to convert output DNs to ambient data for each system.

Table 4. Summary of system temperature calibrations fit values and coefficients

\begin{tabular}{ccc}
\hline System & r-squared fit value & Coefficients $(\boldsymbol{a}, \boldsymbol{b}, \boldsymbol{c})$ \\
\hline 1 & 0.9991 & $1.4981,-277.85,47210.03$ \\
2 & 0.9981 & $1.4928,-268.67,46762.25$ \\
3 & 0.9988 & $1.1894,-289.04,47300.24$ \\
4 & 0.9980 & $1.0850,-289.62,47013.19$ \\
5 & 0.9987 & $1.2189,-290.12,46874.86$ \\
\hline
\end{tabular}

Complete system calibration data are also taken by sweeping the temperature of both the rad-hard circuitry and the sensor board in the environmental chamber so that any drift effects in the output DNs as a result of the wide temperature variation of the rad-hard circuitry can be analyzed and stated project objectives can be met. Results of this test will provide insight into the temperature performance capabilities of the system up to the final digitization of the pulse-width-modulated bit stream output of the sigma-delta modulators.

As depicted by the red data points in Figs. 28 to 32, the drift of the output data as a function of temperature relative to the temperature calibration data (blue) is minimal. In fact, the data sets almost overlap exactly for all five systems. These outcomes are outstanding, considering all of the electrical biasing and functions that are temperature dependent and critical to the accuracy of the system, such as voltage references and current biases, the frequency synthesizer, and input node impedance within the temperature data path. Furthermore, the data verify the wide temperature capabilities of the Triad VCA and FMI frequency synthesizer ICs as well as all other ICs and designed circuitry present on the RHB.

\subsection{PRESSURE CALIBRATION}

Just as with the temperature calibration, a measure of output pressure ADC DN while sweeping input voltage was performed to ensure that the signal conditioning and ADC circuits on the RHB were linear with respect to input. These were preliminary tests to verify linearity of the rad-hard ADCs before moving on to complete system data calibration. Figure 33 shows the results of this input voltage sweep over the entire range of possible pressure sensor output voltages. 


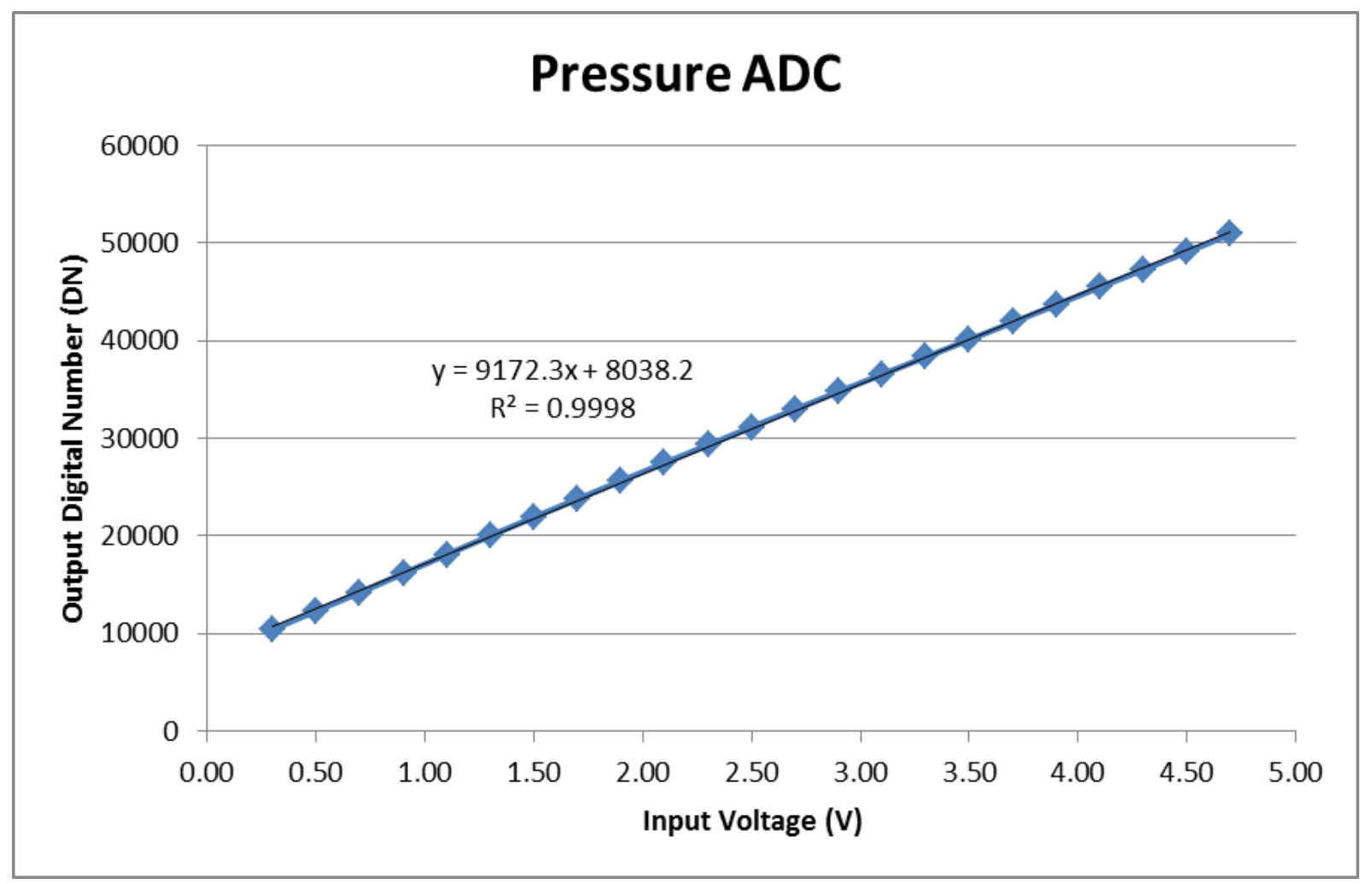

Fig. 33. Preliminary pressure ADC output DN vs input voltage.

The pressure ADC also shows great linearity at an r-squared value of 0.9998 . This is consistent with the preliminary temperature ADC measurement. It is also apparent that the pressure measurement shows an opposite slope as the temperature ADC. This is because the pressure sensor outputs a voltage between 0.5 and $4.5 \mathrm{~V}$. This voltage range is too large for the common mode input range of the ADC, so an inverting gain and shift operational amplifier was implemented to scale the 0.5 to $4.5 \mathrm{~V}$ range down to 1.8 to $3.2 \mathrm{~V}$. This prevents the ADC from receiving voltage levels beyond its input common mode range and outputting invalid data. This scaling is done most easily with an inverting configuration. Thus the pressure signal conditioning and digitization show an inverted behavior with respect to the preliminary temperature ADC test.

The system pressure calibration differs from the system temperature calibration and is expected to be highly linear. This is because the pressure sensor outputs a voltage controlled by internal feedback loop instead of a current; thus, it is not dependent on temperature-sensitive output node impedance. The result of pressure calibration for all five systems is shown in Figs. 34 through 38. The blue data points represent sweeping the pressure input to the pressure sensor in 2.5 psi increments. 


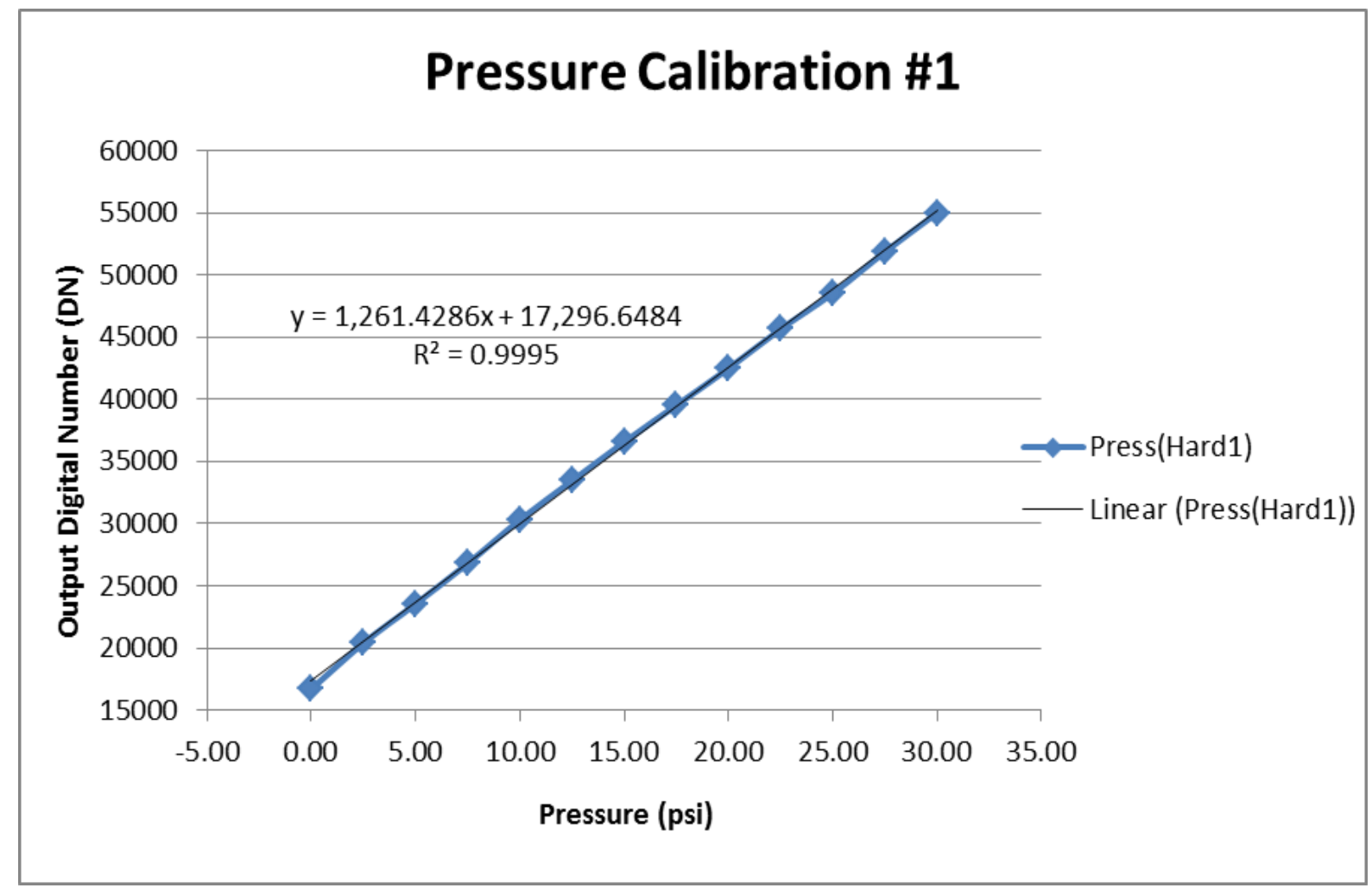

Fig. 34. System 1 pressure output DN vs ambient pressure.

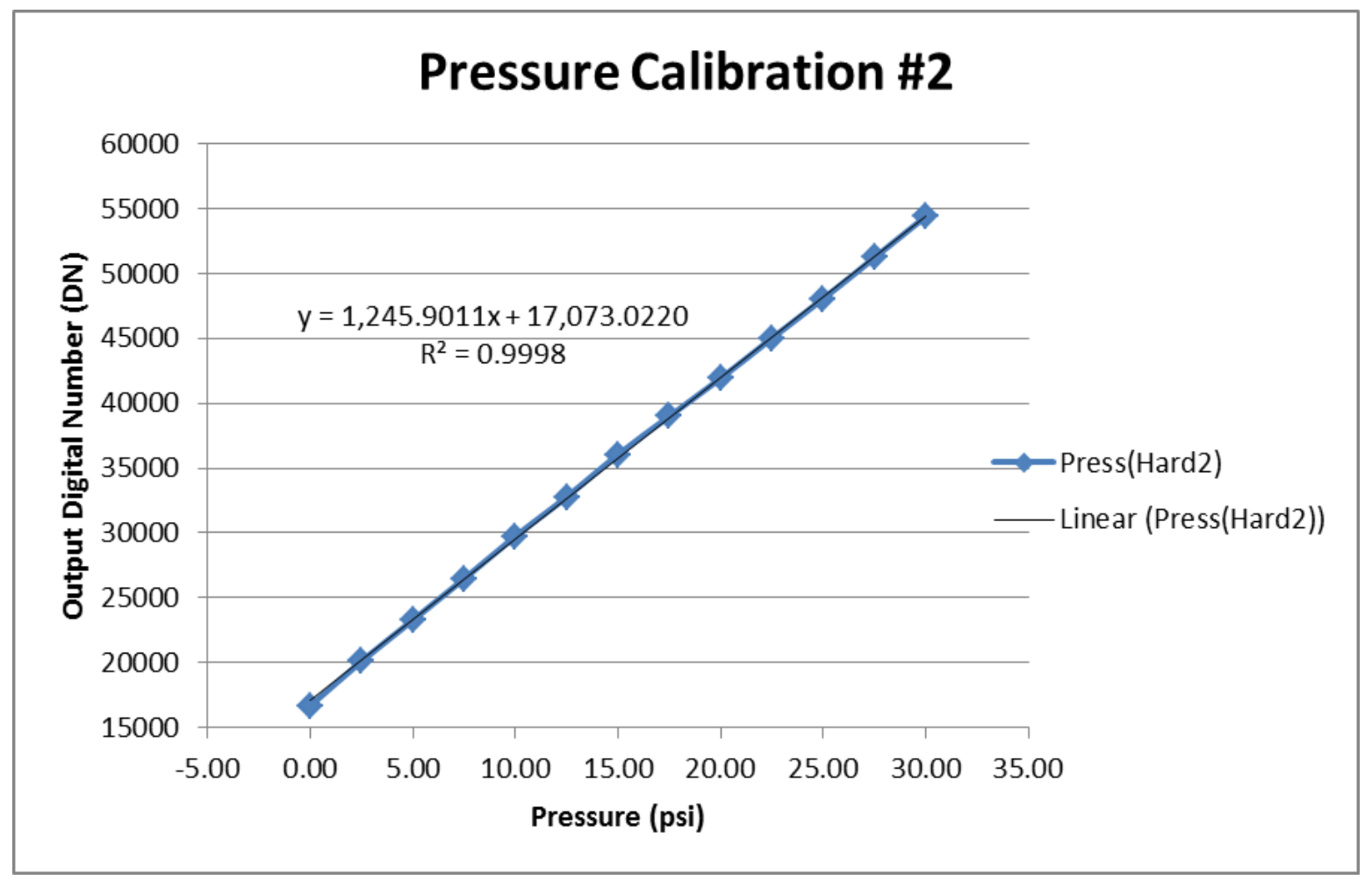

Fig. 35. System 2 pressure output DN vs ambient pressure. 


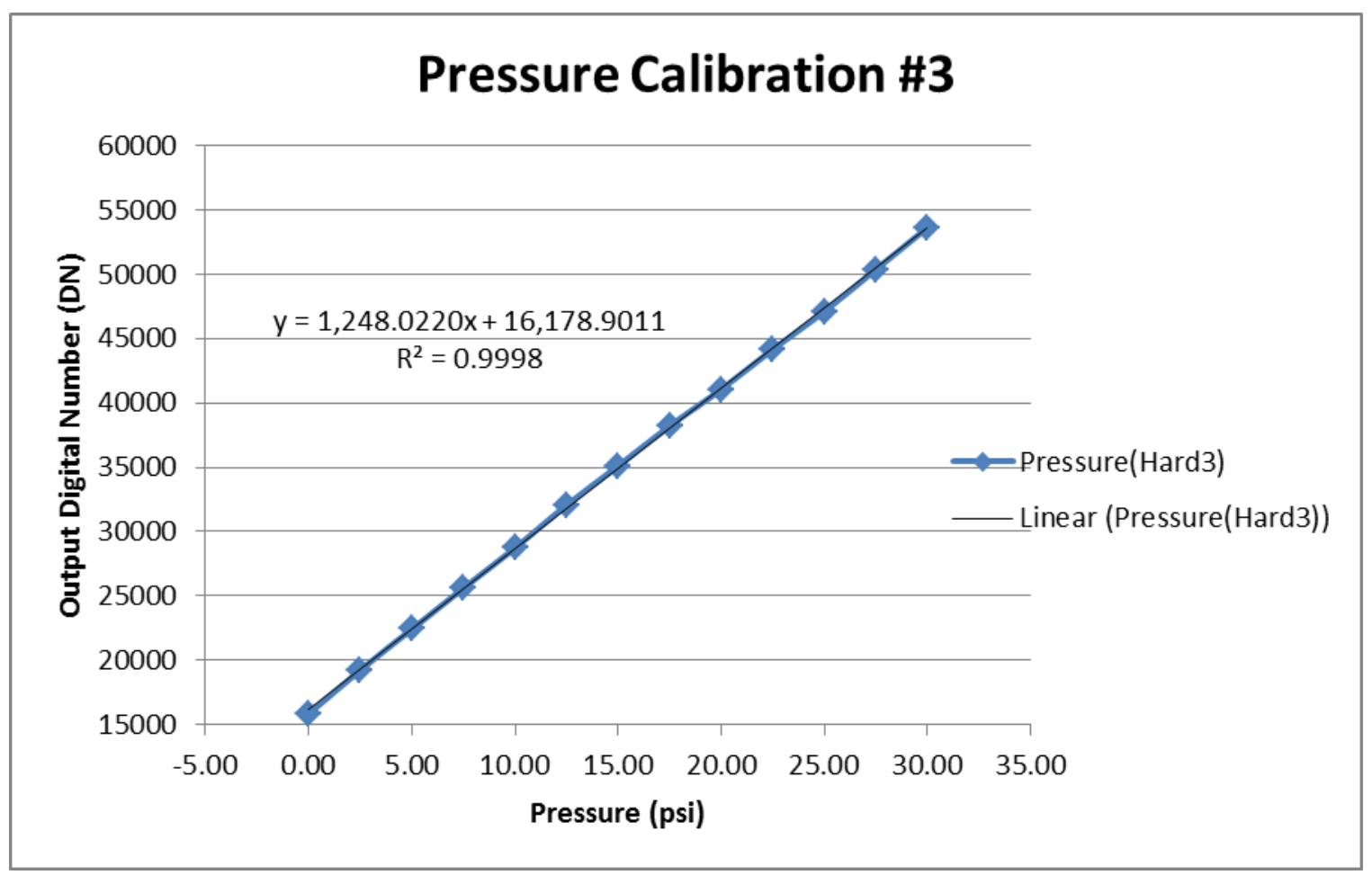

Fig. 36. System 3 pressure output DN vs ambient pressure.

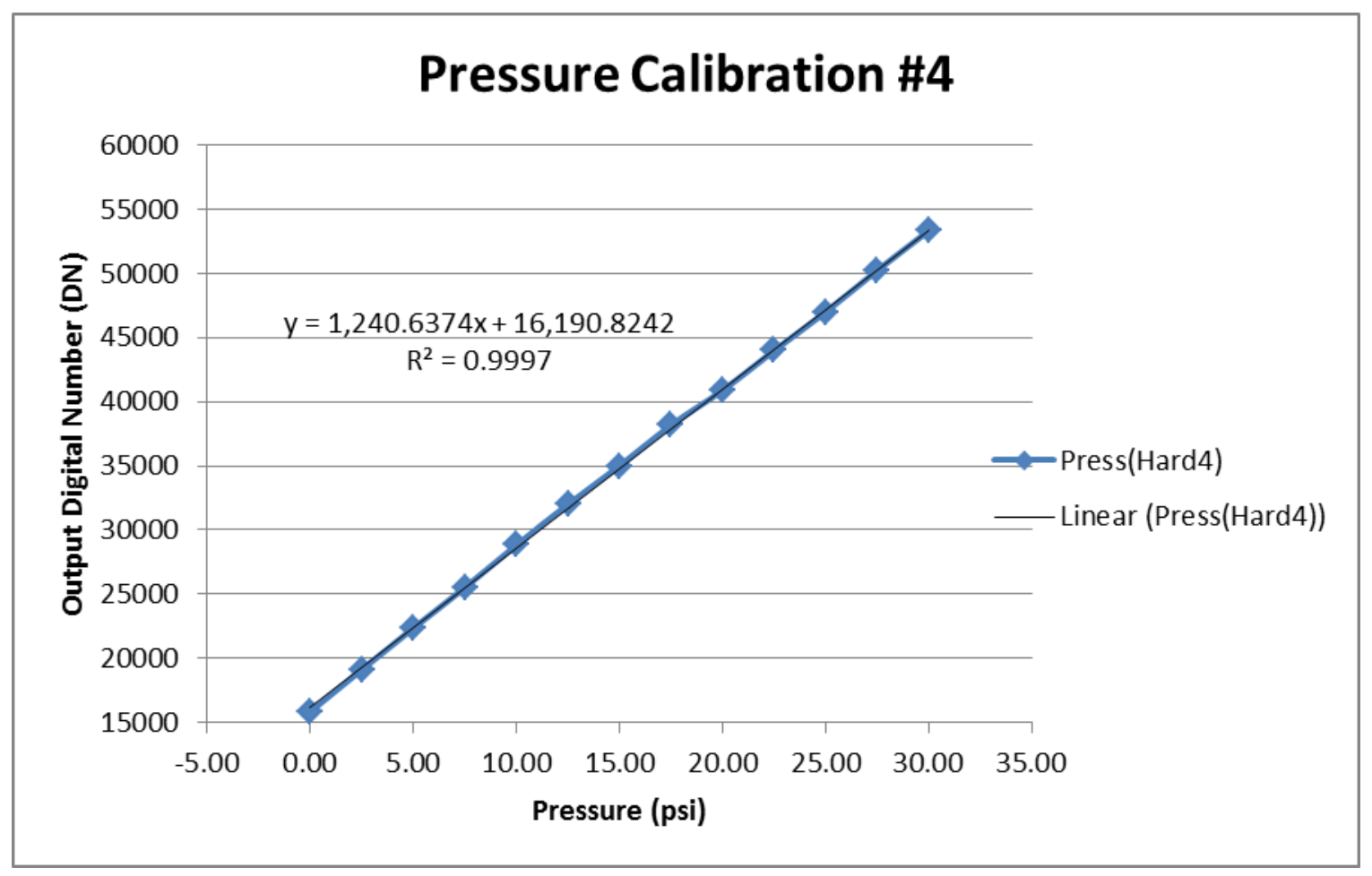

Fig. 37. System 4 pressure output DN vs ambient pressure. 


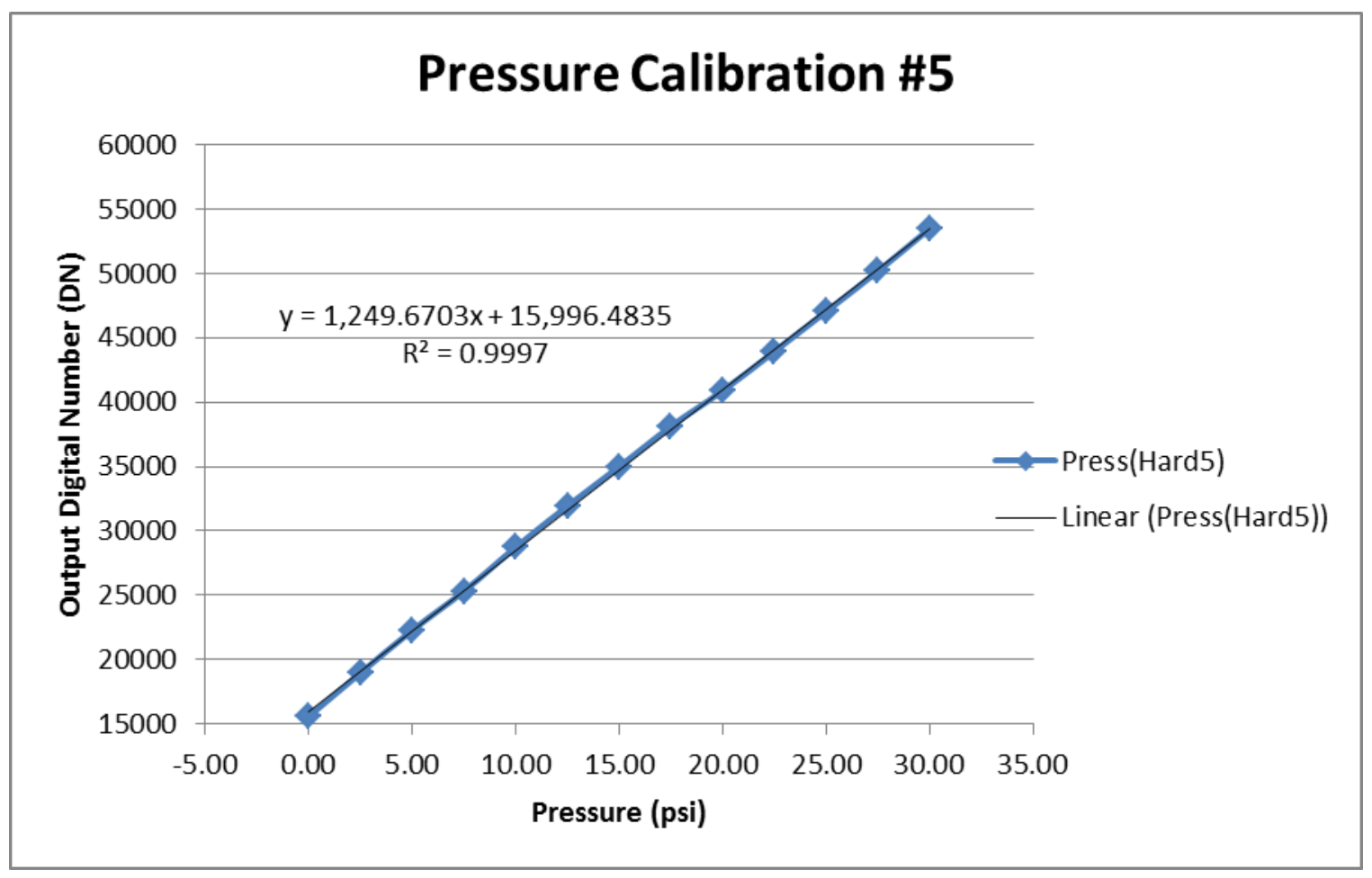

Fig. 38. System 5 pressure output DN vs ambient pressure.

The pressure calibration data for all five systems show great linearity. This result matches the expectations for the pressure sensor and validates the consistency and accuracy of both the pressure sensor and the rad-hard circuitry, making for a simple trendline approximation. A linear fit produces the best approximation for all five systems. Table 5 summarizes for each system the linear r-squared values and coefficients that are used by LabVIEW to convert output DNs to ambient data.

Table 5. Summary of system pressure calibrations fit values and coefficients

\begin{tabular}{ccc}
\hline System & r-squared fit value & Coefficients $(\mathbf{a}, \mathbf{b})$ \\
\hline 1 & 0.9995 & $1261.429,17296.65$ \\
2 & 0.9998 & $1245.901,17073.02$ \\
3 & 0.9998 & $1248.022,16178.90$ \\
4 & 0.9997 & $1240.637,16190.82$ \\
5 & 0.9997 & $1249.670,15996.48$ \\
\hline
\end{tabular}

\subsection{GAMMA DETECTION CALIBRATION}

The gamma detection calibration is different in nature from both the temperature and pressure calibrations in that the charge generated by the event is only present for a short period of time, and it needs to be classified. The current spike output from the G-M tube is converted to voltage through a resistor and, depending on the peak level of current, the voltage will also reach a certain peak value. In order to discern whether a voltage spike is in fact a radiation event, a certain voltage threshold must be met. This can be achieved by comparing the voltage spike with a reference voltage that is unachievable by any excitation other than a radiation event. If the voltage spike surpasses the threshold, the comparator will output a digital high value, representing one event, and subsequently will return to a digital low in preparation for 
the next event. These events can be summed with an FPGA digitally implemented counter, which will notify users of the total ionizing dose as well as the dose rate, which is derived through the rate of change of the event count.

The first step in calibrating the gamma detection performance is optimizing the G-M tube output voltage amplitude for compatibility with the system. The comparator operates on a $5 \mathrm{~V}$ supply and could be damaged if voltage spikes larger than $5 \mathrm{~V}$ are present on its input. A potentiometer is used to scale the current-to-voltage conversion down to the necessary amplitude of about $4.5 \mathrm{~V}$. This waveform was captured on an oscilloscope and is shown as the yellow spike in Fig. 39.

The next step is to ensure that the comparator exhibits sufficient rise and fall times to record every gamma event output from the G-M tube without overlap. Maximizing the gain bandwidth of the comparator through all available parameters will produce the fastest rise and fall times possible for a given comparator design. In this case, we have control over the input bias current, which was increased to a maximum $150 \mu \mathrm{A}$. The result is a $30 \mathrm{~ns}$ rise time, shown in Fig. 39 as the green waveform. The maximum event rate of the G-M tube is $50,000 \mathrm{cps}$, which is equivalent to $20 \mu \mathrm{s}$ per count. This means that the comparator needs to produce a digital high value and return to a digital low within $20 \mu$ s of the initial gamma event pulse. Figure 39 shows that the comparator easily achieves this requirement, with about a $600 \mathrm{~ns}$ pulse width and only about $800 \mathrm{~ns}$ until the output completely settles back to digital low. With these specifications, the comparator, assuming the voltage threshold reference is set properly, will capture every gamma event.

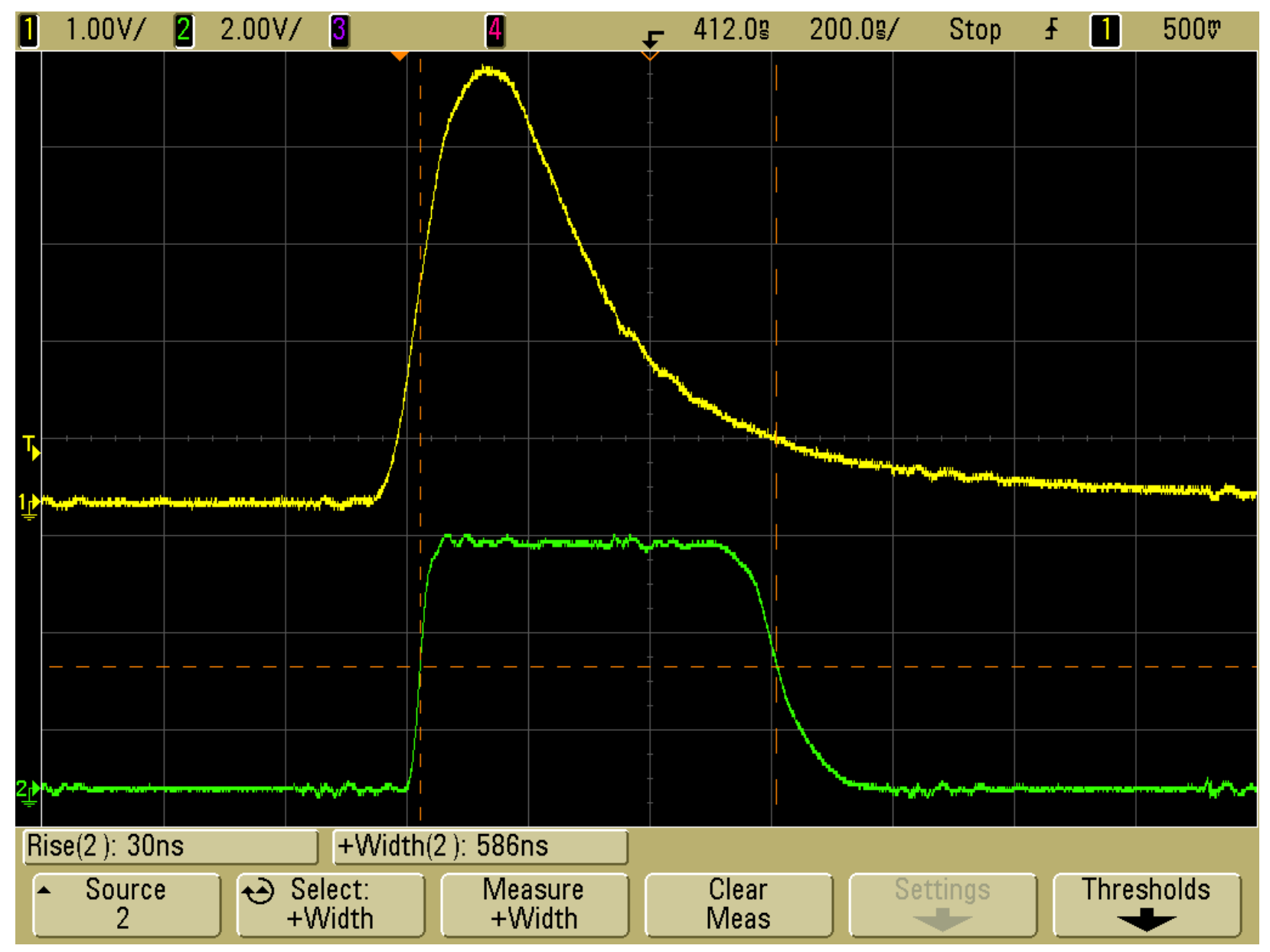

Fig. 39. Gamma event and comparator output pulse response. 
The voltage threshold for the comparator must be optimized to allow classification of each gamma event but to reject any other voltage spikes (e.g., spikes that may be induced by noise or incident radiation). Because the G-M tube functions based on an avalanche effect and produces a large amount of charge, the potentiometer resistance must be set relatively low in order to achieve the $4.5 \mathrm{~V}$ pulse shown in Fig. 39. At such a low resistance, it is unlikely that any voltage spikes from charge deposition, other than those produced by the G-M tube, will result in a signal with an amplitude larger than $0.5 \mathrm{~V}$. Thus the voltage threshold reference for the comparator can safely be set to $0.5 \mathrm{~V}$ to produce large enough pulse widths for the counter to recognize while also discarding any unwanted, spurious signals.

\section{POST-IRRADIATION PERFORMANCE TESTING}

For circuits that are not protected from TID radiation, severe nonideal behavior can result as a function of increasing total radiation dose. These effects include shifts in device threshold voltages, increased leakage currents, and uncharacteristic extreme variation in circuit performance. In modern submicron processes, voltage threshold variations have been almost completely mitigated due to reduced gate oxide thickness and charge-trapping ability. However, leakage currents and performance variations still pose issues, and circuits designed for radiation hardness need to be verified against these effects.

Annular gate and guard ring layout techniques are popular methods for mitigating increased leakage currents and thus extreme performance variation. These techniques are featured in the Triad VCA and FMI frequency synthesizer ASICs used for analog-to-digital conversion on the RHB. Consequently, the expectation for leakage current and system performance variations as a function of TID radiation is minimal. Although, as seen in the temperature calibration data, if any radiation chamber temperature change induces a shift in node resistance on the output of the temperature sensor, some slight nonlinear increase in data will be apparent. Significant increases in leakage current will manifest in system current consumption data since a large portion of the system's power expenditure is on the RHB itself. It is also likely that severe leakage currents will induce changes in circuit quiescent points and will be apparent in the system performance data shown in Figs. 41 to 52.

For the purposes of radiation testing, three complete systems (systems 1, 4, and 5) underwent radiation exposure within the Gammacell 220. This is enough of a sample size to effectively analyze the radiation tolerance of the system while also preserving two systems (systems 2 and 3) for any future pre-radiation uses. System 5 was irradiated to a total dose of $200 \mathrm{krad}$, and the remaining two systems, systems 1 and 4 , were irradiated to a total dose of $300 \mathrm{krad}$. The project proposal [1] stated a requirement of only $200 \mathrm{krad}$ total dose exposure for each system, but typical radiation "tolerant" qualification for circuitry is at the threshold of $300 \mathrm{krad}$ total dose, and this qualification is useful for making claims about the system in conference or journal report submissions. Figure 40 shows an image of the RHB inside the containment chamber before being lowered into the gamma radiation pool at test commencement. 


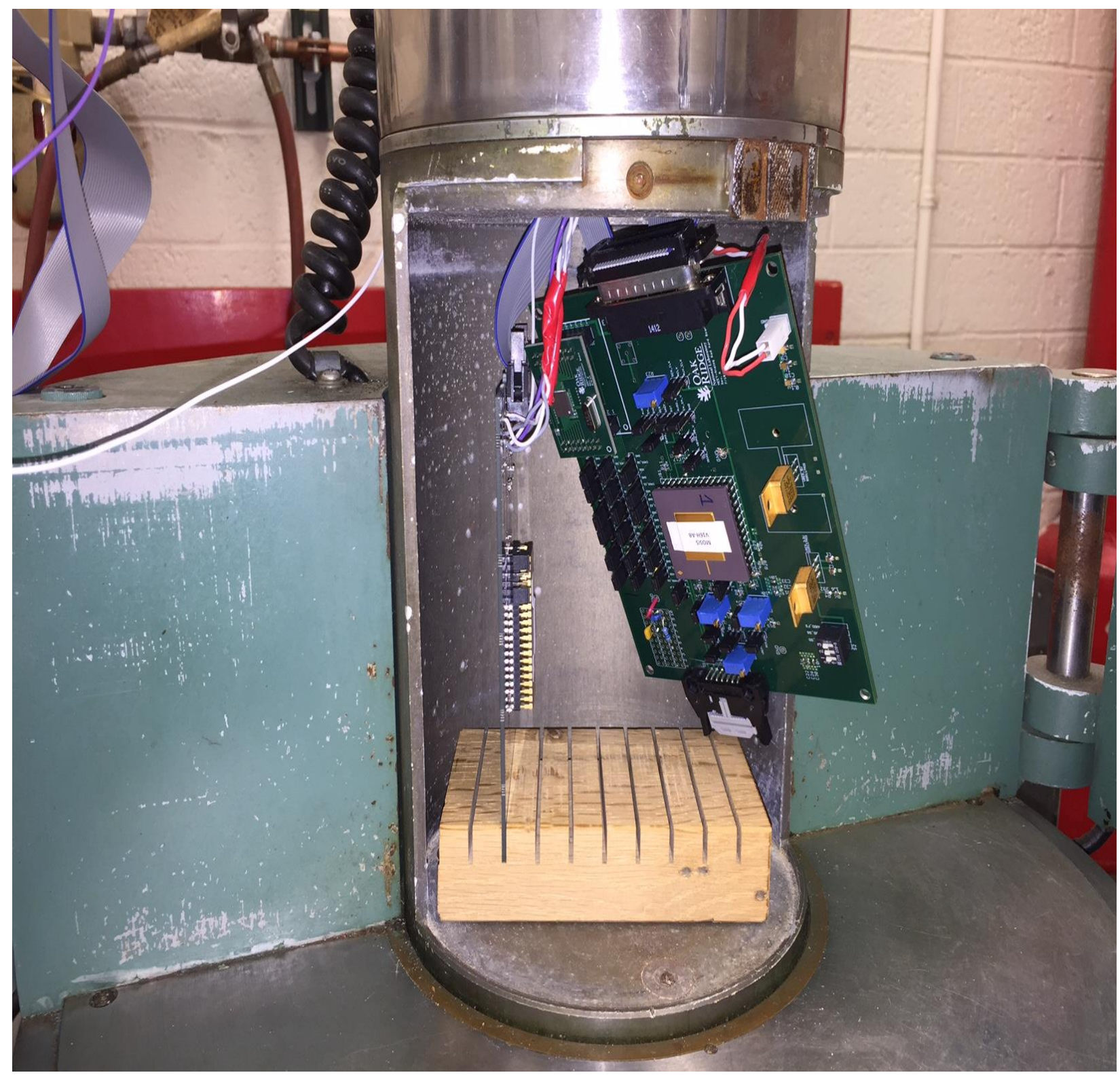

Fig. 40. Rad-hard board setup before test commencement.

\subsection{CURRENT CONSUMPTION RADIATION PERFORMANCE}

The first parameter susceptible to radiation effects to consider is leakage current. Significant increases in device leakage current will produce large changes in the current consumption of the system. Therefore, values of system current consumption were recorded for all three irradiated systems every hour throughout the duration of the test. The recorded measurements for system 1 (Fig. 41) show a small dip in current consumption approaching the midpoint of the test and a slight increase during the latter portion of the test but not beyond the predose measurement value of $142.88 \mathrm{~mA}$. System 4 measurements (Fig. 42) reveal only just resolvable variation throughout the duration of the test, beginning at $150.69 \mathrm{~mA}$ and ending at $151.28 \mathrm{~mA}$. System 5 measurements (Fig. 43) depict a slow, consistent decrease in current consumption of about $13 \mu \mathrm{A} / \mathrm{krad}$ until the $200 \mathrm{krad}$ stopping point. The maximum percent errors from predose measurement for systems 1,4 , and 5 are $1.64 \%, 0.45 \%$, and $1.71 \%$, respectively. Not enough variation in current biasing has occurred to induce shifts in device quiescent points. All of these small 
variations signify large amounts of excess charge being generated and escaping the circuit nodes by electric field through the power supply terminals but do not represent induced leakage current effects as a function of TID radiation. Moreover, at these levels of percent error, the radiation hardness of the exposed circuitry is proven with respect to radiation-induced leakage current.

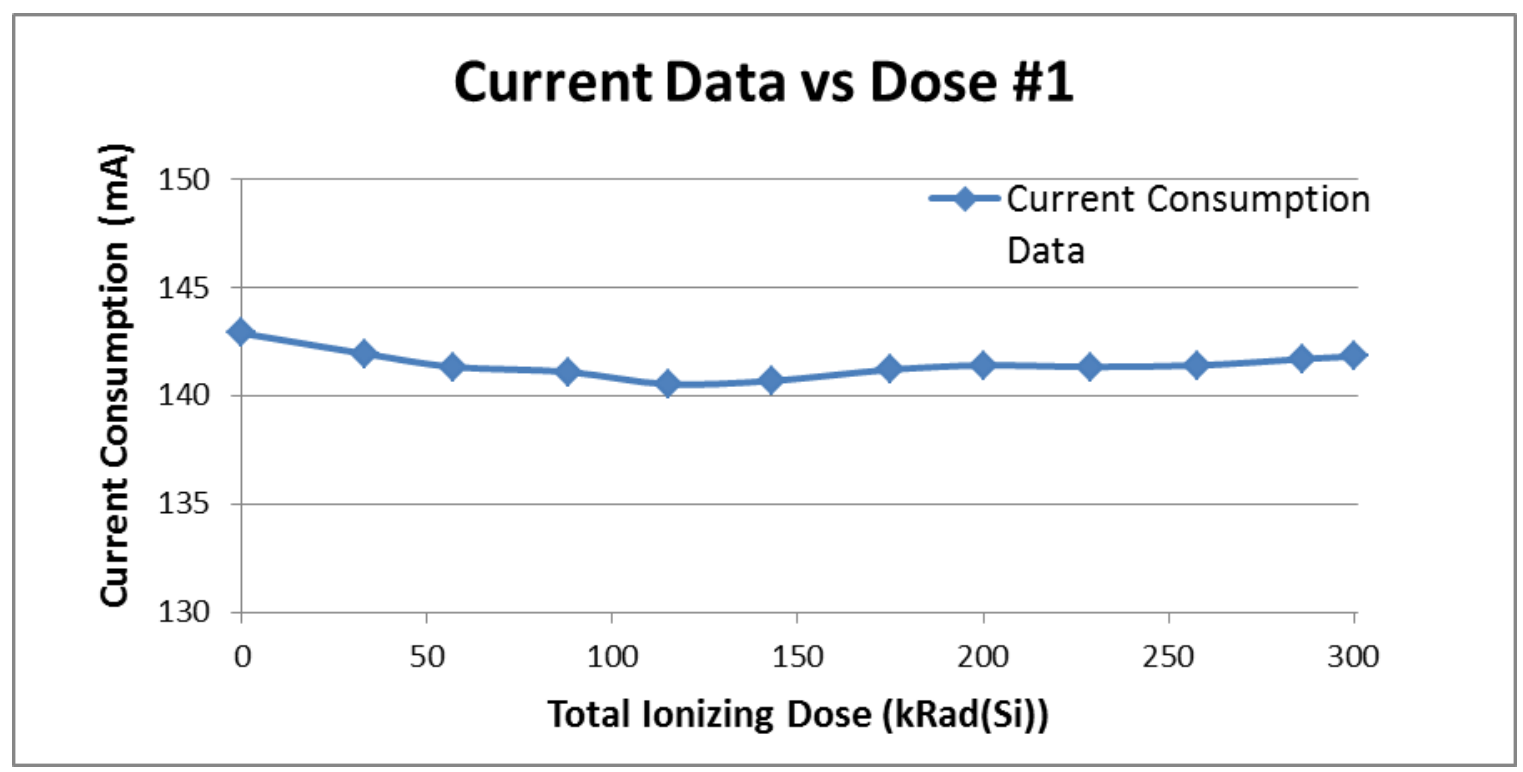

Fig. 41. System 1 current consumption vs TID radiation.

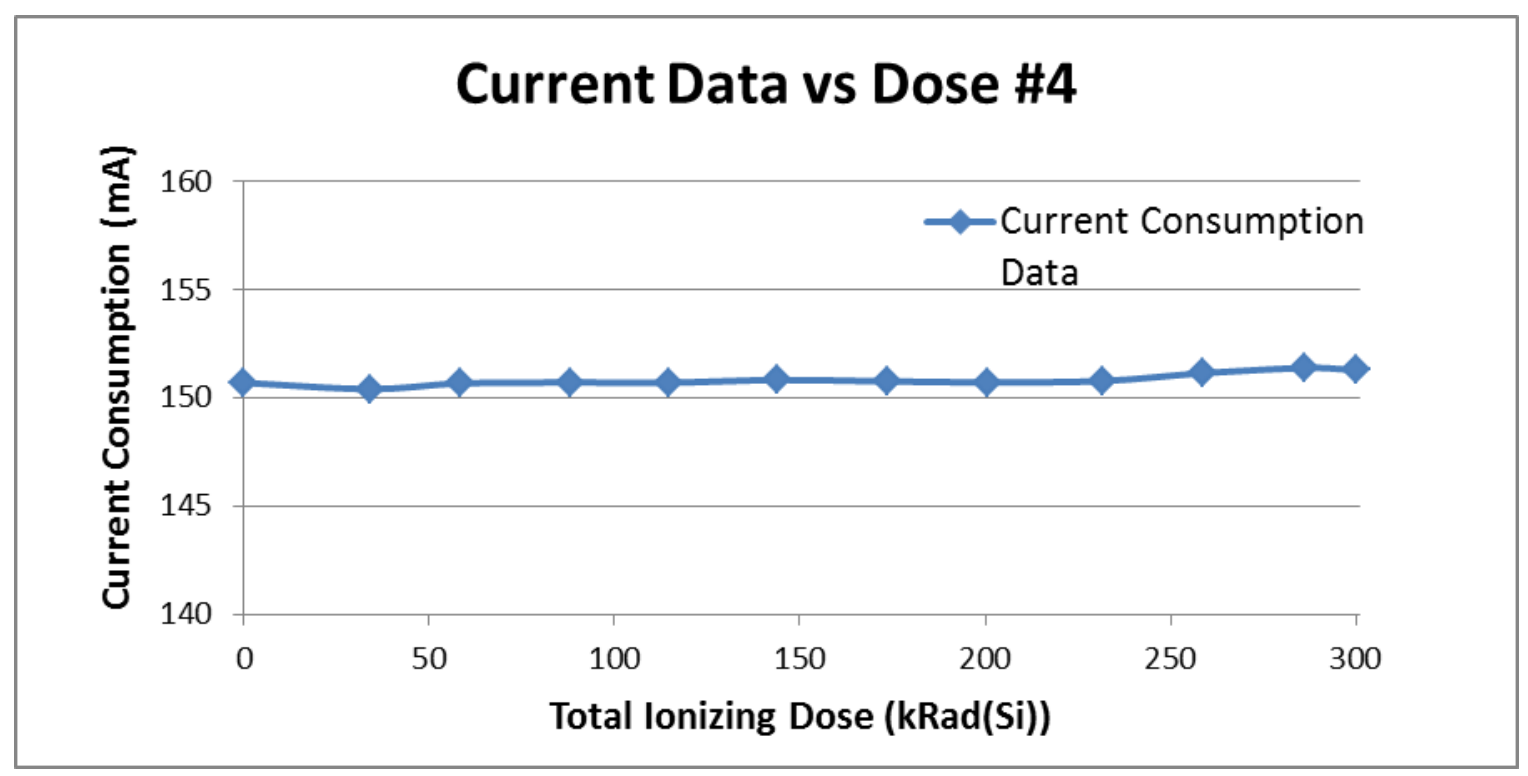

Fig. 42. System 4 current consumption vs TID radiation. 


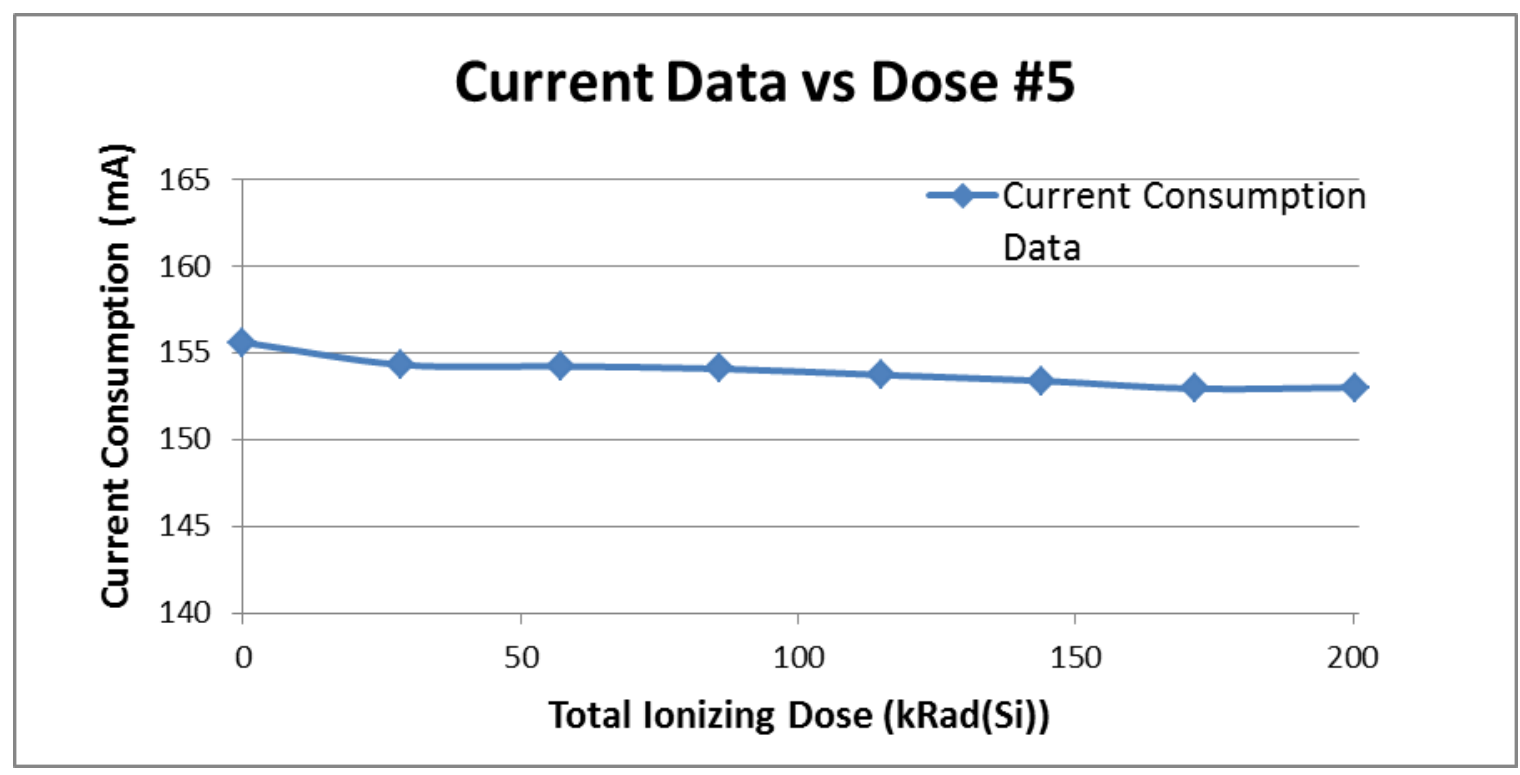

Fig. 43. System 5 current consumption vs TID radiation.

\subsection{TEMPERATURE DATA RADIATION PERFORMANCE}

After leakage current effects are determined to not be a detriment to the performance of the data acquisition system, the actual ambient data output from the system needs to be examined as a function of radiation. The LabVIEW software recorded ambient temperature output data once every 10 cycles. This was done to prevent excessive amounts of data from being taken but also to gather enough data for interpretations such as noise analysis to be performed. In addition, it is known that temperature sensors typically exhibit some initially unknown offset when compared to real temperature unless trimming is implemented using additional devices. The data are highly useful even without trimming because trends can be seen. During severe accident scenarios, trends are more important than actual temperatures.

The graphs of Figs. 44 to 46 relate the data acquisition system temperature data to ambient room temperature as a function of radiation. All three systems show a temperature data slope slightly more positive than the slope of the ambient room temperature. The larger slope of the temperature data is attributed to heating of the radiation chamber, in which radiation and heat from power dissipation in a small space are contributors, that induces inherent resistance shifts on the temperature sensor current output node, resulting in a voltage change. This is consistent with all three systems; even though the ambient temperature for system 5 was actually decreasing, system 5 also exhibits the least positive slope of all systems irradiated. The offsets of each sensor are also independently apparent, as two system's predose temperature data were below the room temperature, while the other system shows temperature data initially above the room temperature curve. By factoring out the effects of temperature sensor offset error and output node resistance temperature sensitivity, which are both due to the temperature sensor performance itself, the mask-programmable analog array can closely replicate ambient temperature data within a data acquisition system for nuclear reactor environment applications under extreme radiation conditions. 


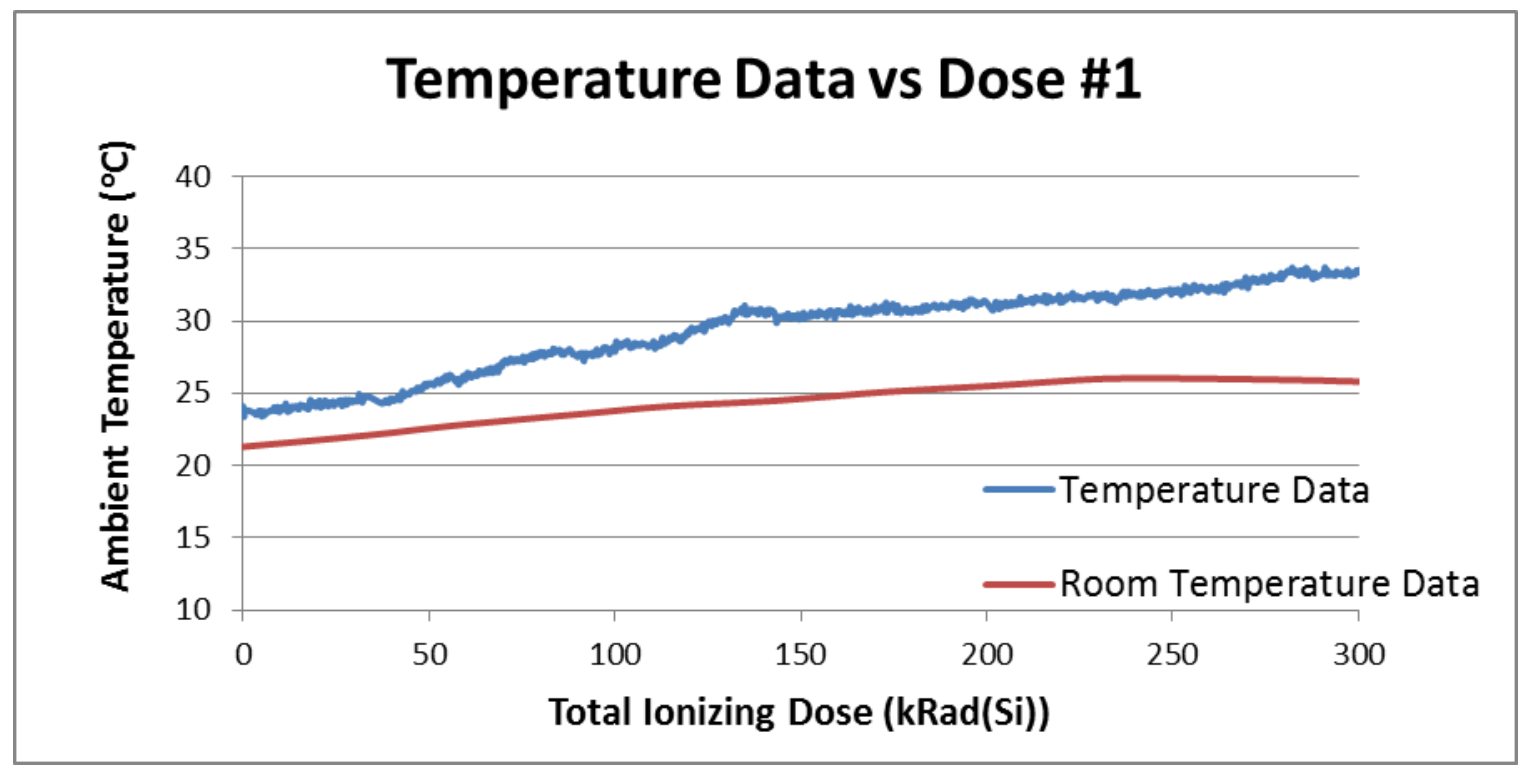

Fig. 44. System 1 temperature reading vs TID radiation.

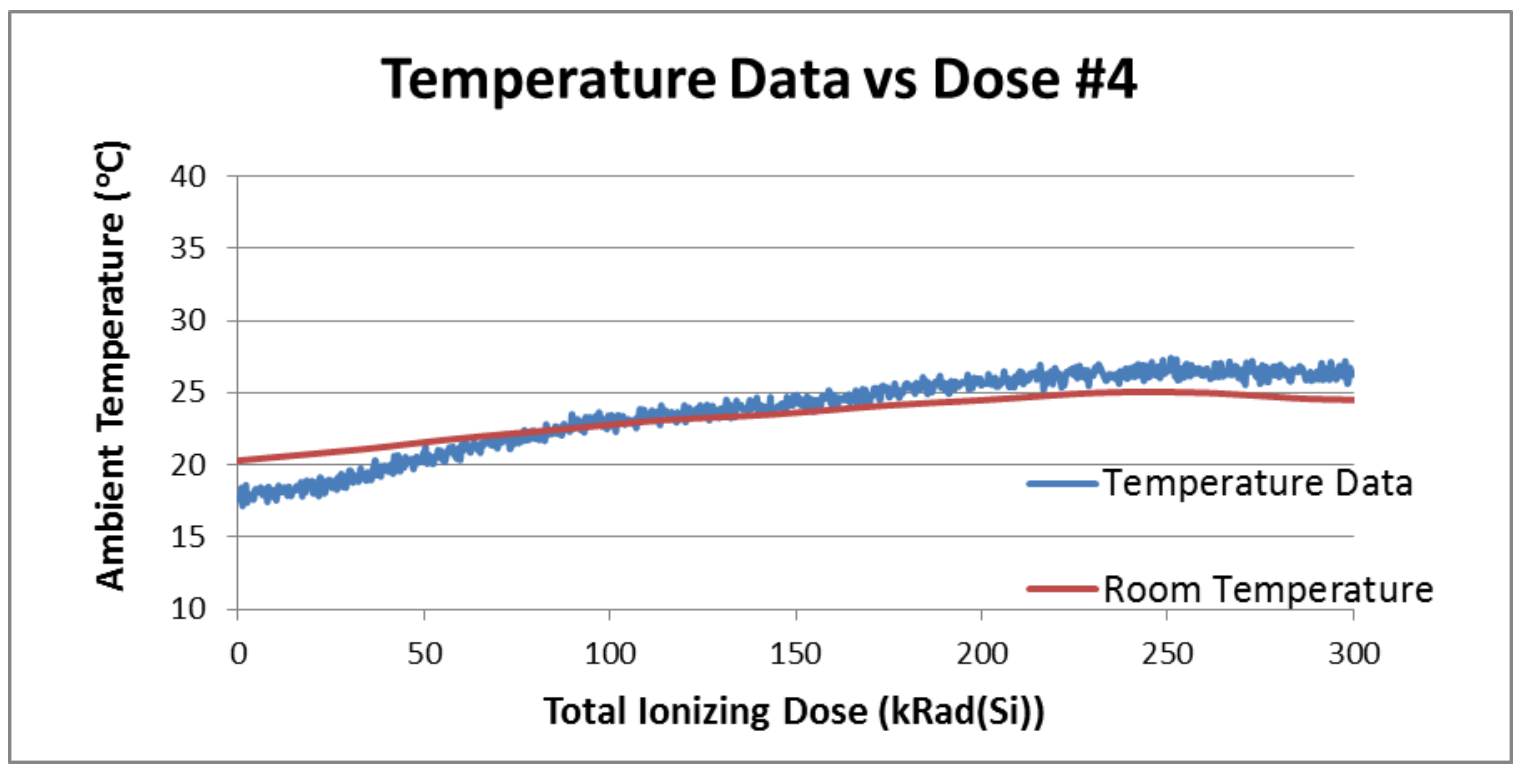

Fig. 45. System 4 temperature reading vs TID radiation. 


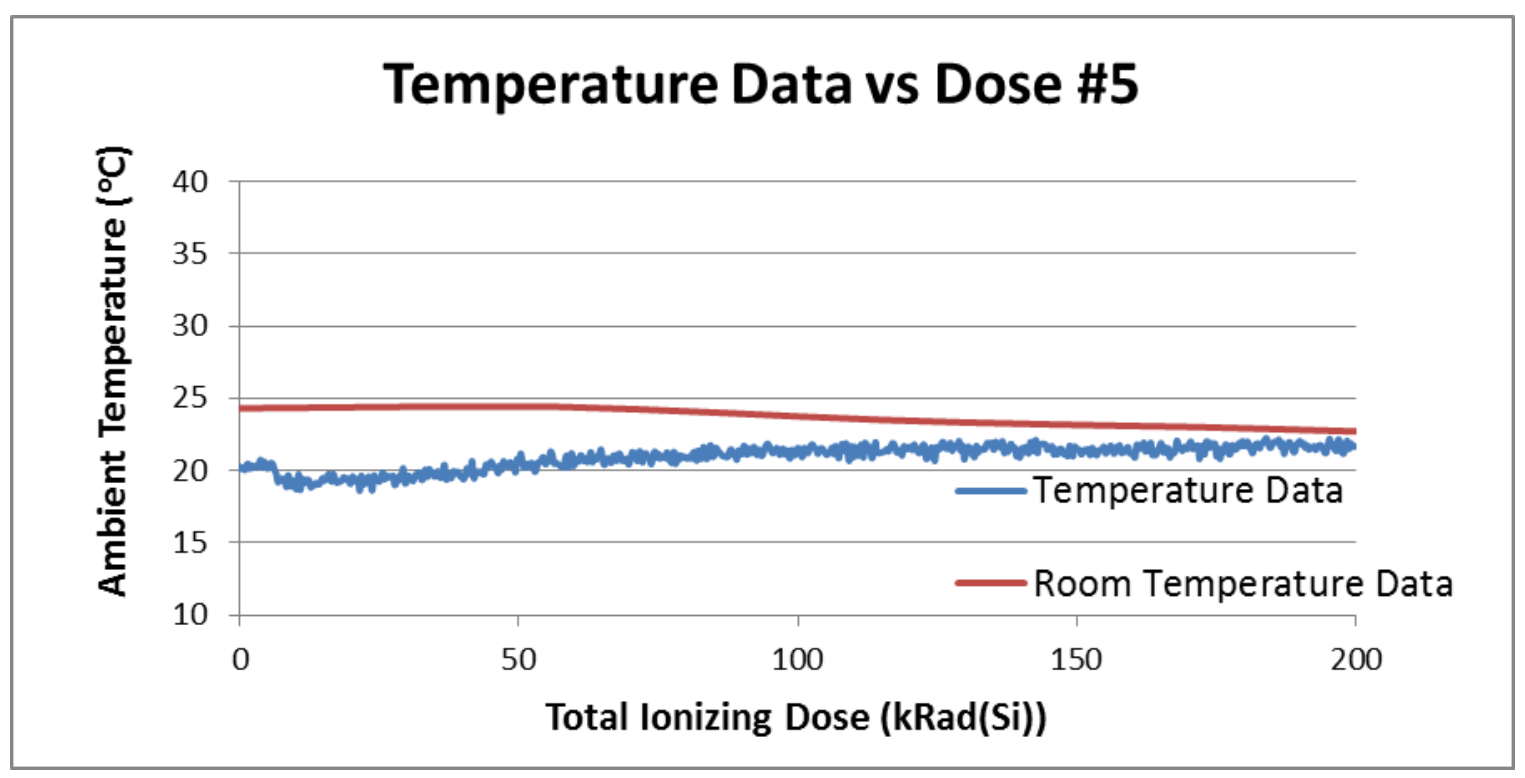

Fig. 46. System 5 temperature reading vs TID radiation.

\subsection{PRESSURE DATA RADIATION PERFORMANCE}

As with the data acquisition system temperature radiation data, the LabVIEW software recorded ambient pressure output data once every 10 cycles. Since the pressure sensor selected outputs a voltage that is closely controlled by an internal feedback loop and compensated against temperature and nonlinear effects, the output itself is not dependent on any other ambient parameter it will be exposed to, except pressure. This fact bodes well for the accuracy in performance of the data acquisition system pressure data as a function of radiation without the need to factor out any nonideal effects. The same sigma-delta modulator digitizes both the analog temperature and pressure data, so this improvement in sensor tolerance to atmospheric nonlinear effects should result in enhanced accuracy and stability relative to the temperature radiation data.

The graphs of Figs. 47 to 49 display the data acquisition system pressure data for systems 1, 4, and 5, respectively. All three systems show extremely consistent pressure data centered at about 0.3 psi, relative pressure. They also exhibit highly stable pressure data whose variation is only just visibly resolvable. The maximum percent errors from predose measurements for systems 1, 4, and 5 are $4.51 \%, 2.66 \%$, and $2.9 \%$, respectively. A significant portion of that maximum percent error for each system is due to noise variations in the pressure data, although the pressure data noise is noticeably smaller in magnitude relative to the temperature data variations. Nonetheless, the pressure data exemplify the performance capabilities of the mask-programmable analog array in radiation-rich environments such as the data acquisition system for applications such as nuclear reactor environment monitoring as well as the improvement in authenticity of reported ambient data with the use of sensors qualified for specific implementations. 


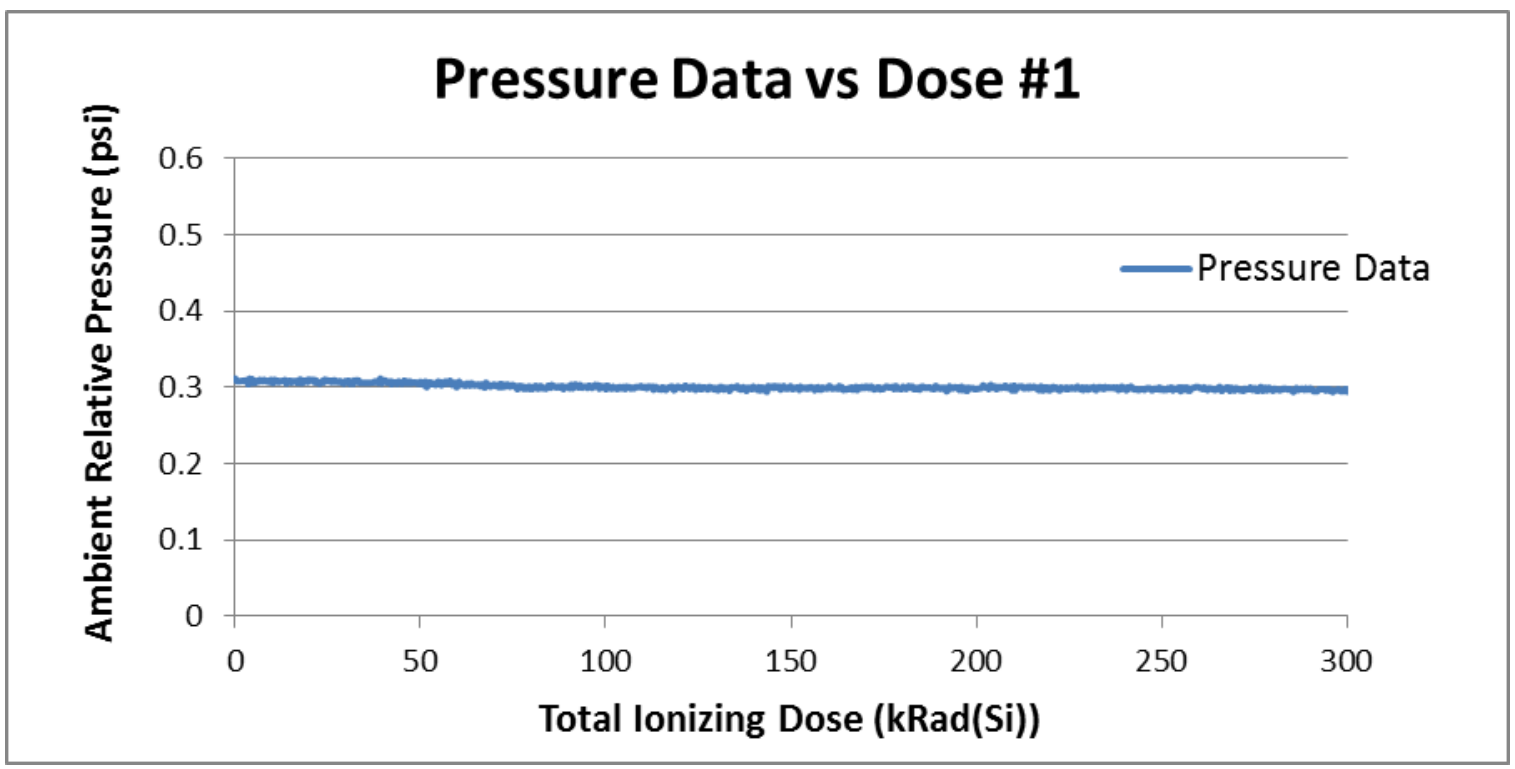

Fig. 47. System 1 pressure reading vs TID radiation.

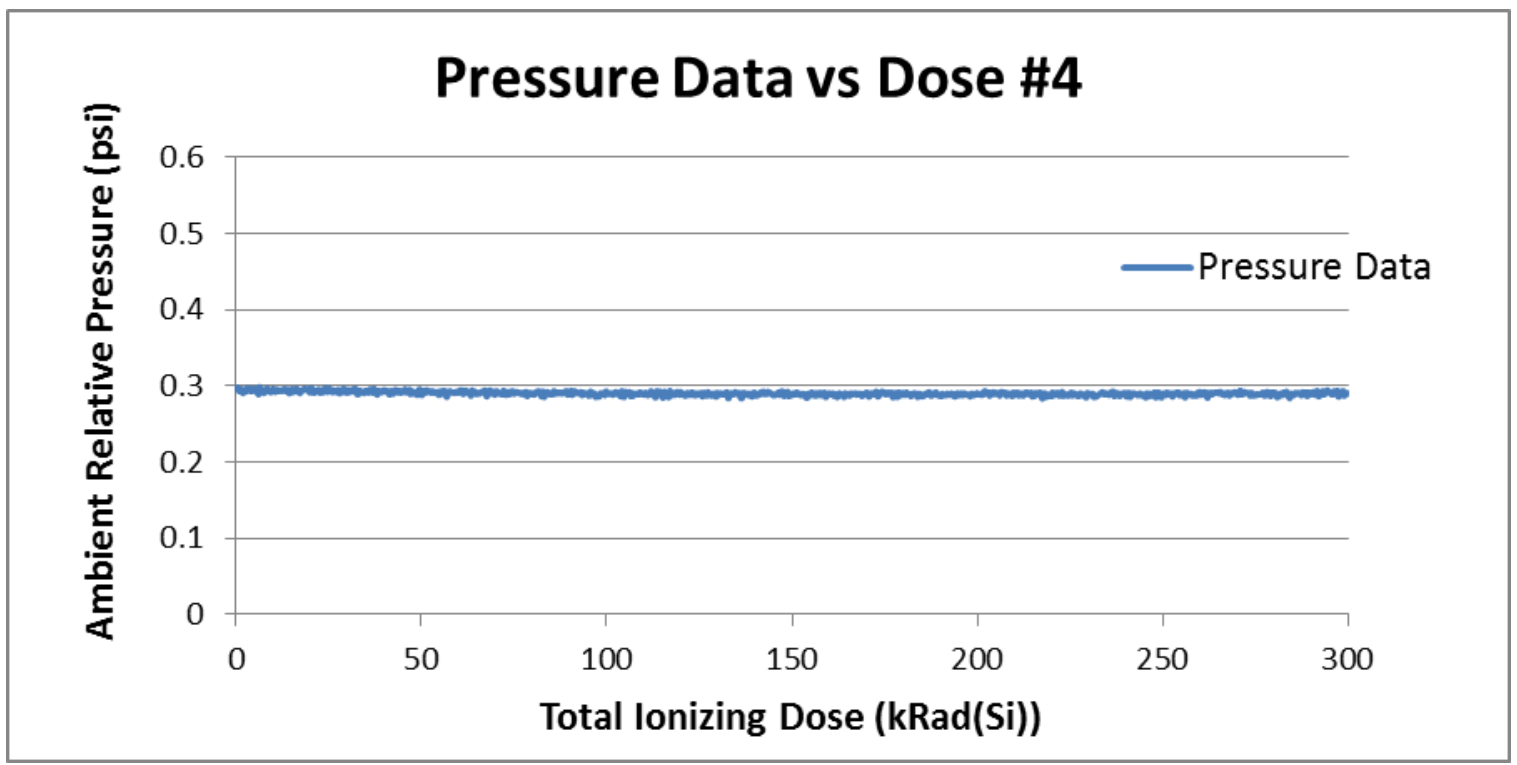

Fig. 48. System 4 pressure reading vs TID radiation. 


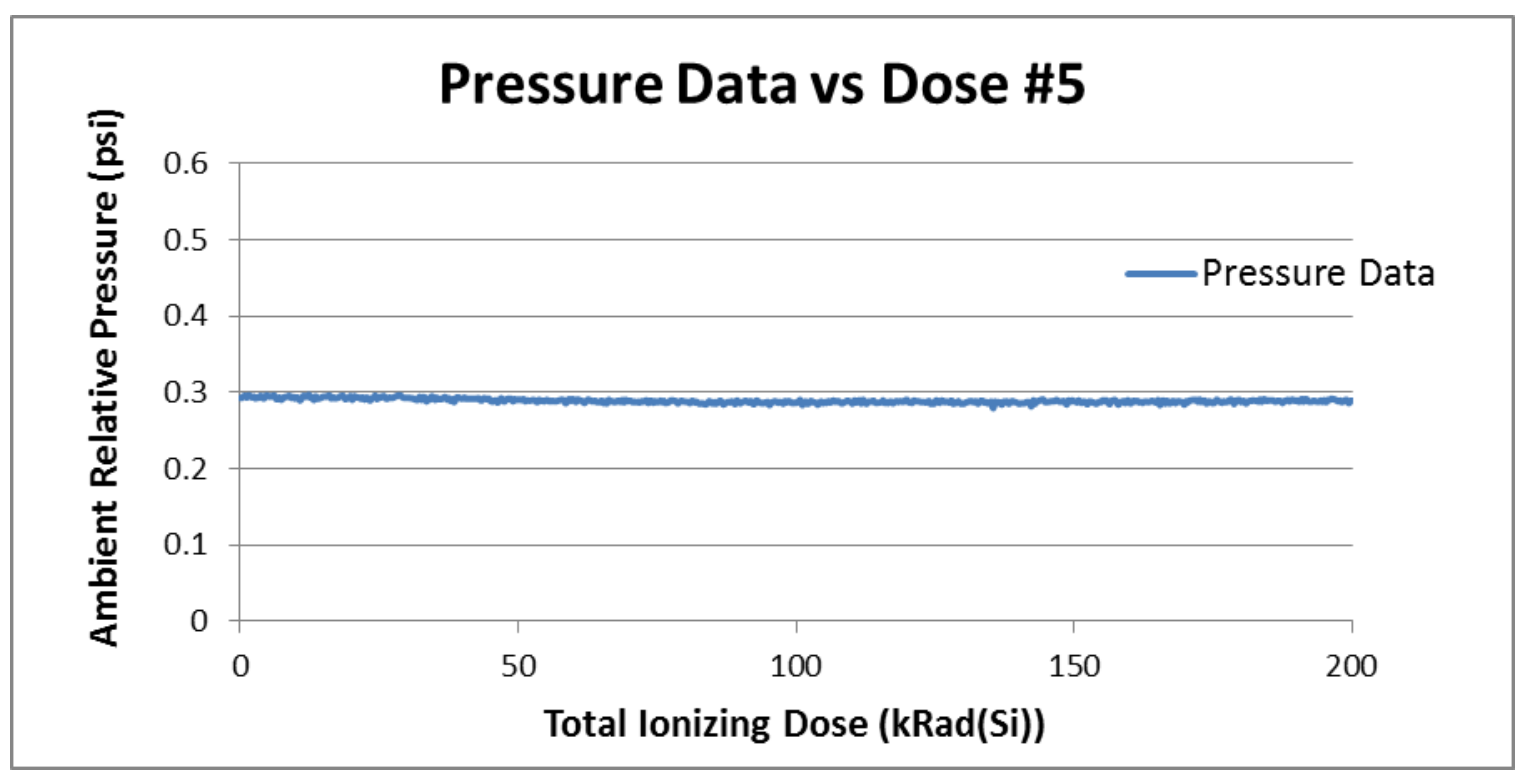

Fig. 49. System 5 pressure reading vs TID radiation.

\subsection{GAMMA EVENT DATA RADIATION PERFORMANCE}

The data gathering rate for gamma events was the same as that for the temperature and pressure parameters. The LabVIEW software recorded ambient pressure output data once every 10 cycles. The G-M tube used for gamma radiation detection is capable of measuring up to 50,000 gamma counts per second, which saturates at roughly $400 \mathrm{rad} / \mathrm{hr}$ from cobalt- 60 . This rate is well above typical background radiation levels, so any significant count errors reported due to radiation exposure of the rad-hard electronics will be visible in the gamma event data. Since the gamma events are singular in nature and require digital electronics such as comparators and counters to be quantified, it is unlikely that TID radiation will induce populous bit count errors, but it is possible.

Normalized gamma events in units of counts per second were counted, calculated, and then plotted for systems 1, 4, and 5 in Figs. 50, 51, and 52, respectively. These plots show a seemingly random distribution of single gamma counts throughout the duration of the test. Nearly all of the relevant readings show about $0.3 \mathrm{cps}$, which equates to one gamma count in slightly less than a $4 \mathrm{~s}$ data gathering cycle. System 4 registered nothing higher than $0.3 \mathrm{cps}$ during the entire duration of the test. System 5 reported two 0.6 cps cycles (two gamma counts slightly less than a $4 \mathrm{~s}$ cycle). System 1, in particular, shows a series of $0.6 \mathrm{cps}$ cycles between the $100 \mathrm{krad}$ and $150 \mathrm{krad}$ TID radiation range. The data for system 1 are slightly peculiar but do not signify any count error due to radiation effects; an event rate of $0.6 \mathrm{cps}$ still equates to background radiation levels, especially in a small laboratory containment housing multiple radiation sources. The data soon returned to the $0.3 \mathrm{cps}$ standard level for these systems. Acquisition of these data concludes radiation tolerance testing for the data acquisition system, and the data confirm the ability of the mask-programmable analog array to condition and digitize analog signals accurately and efficiently in the presence of harsh radiation environments such as nuclear reactor containments. 


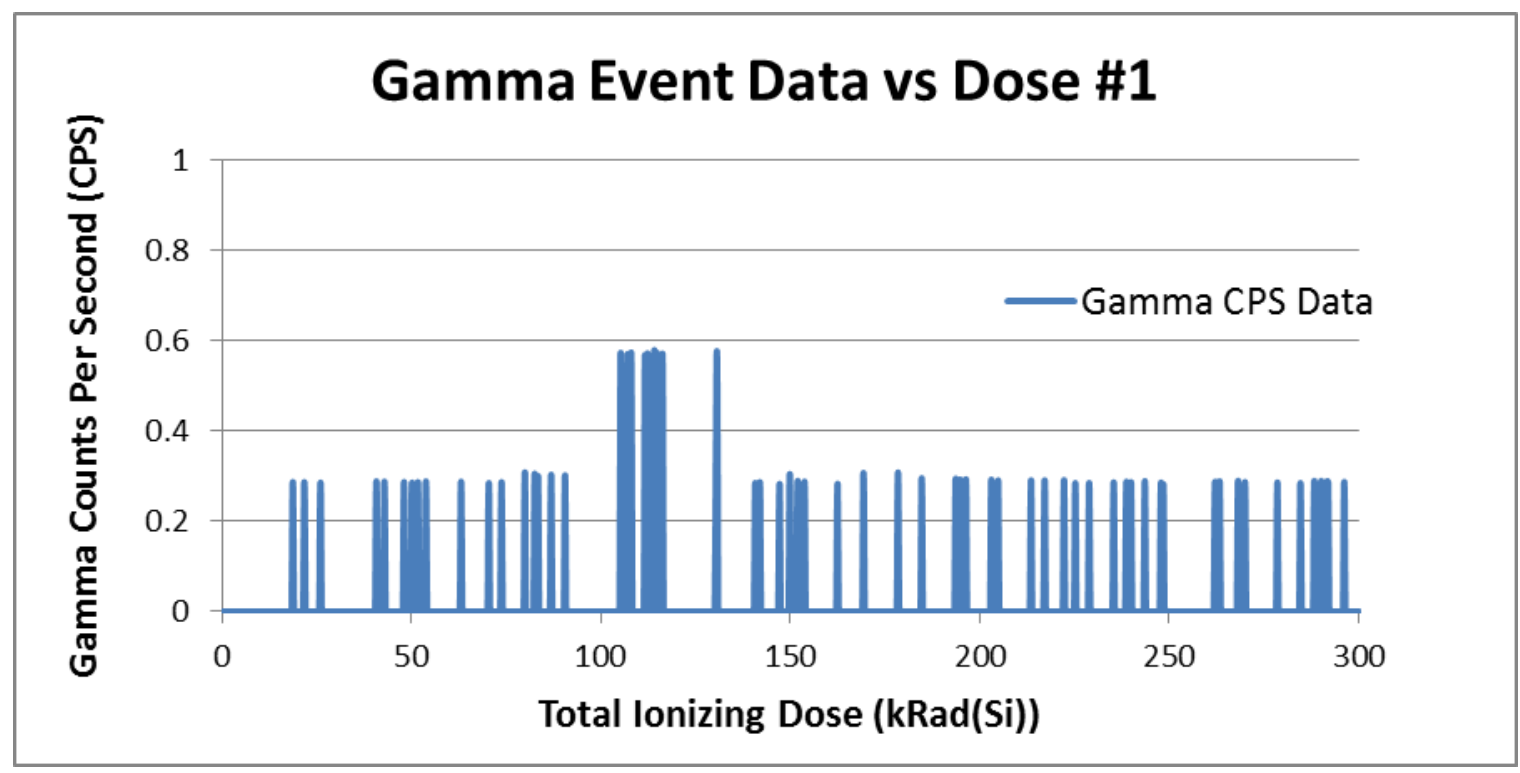

Fig. 50. System 1 gamma count rate vs TID radiation.

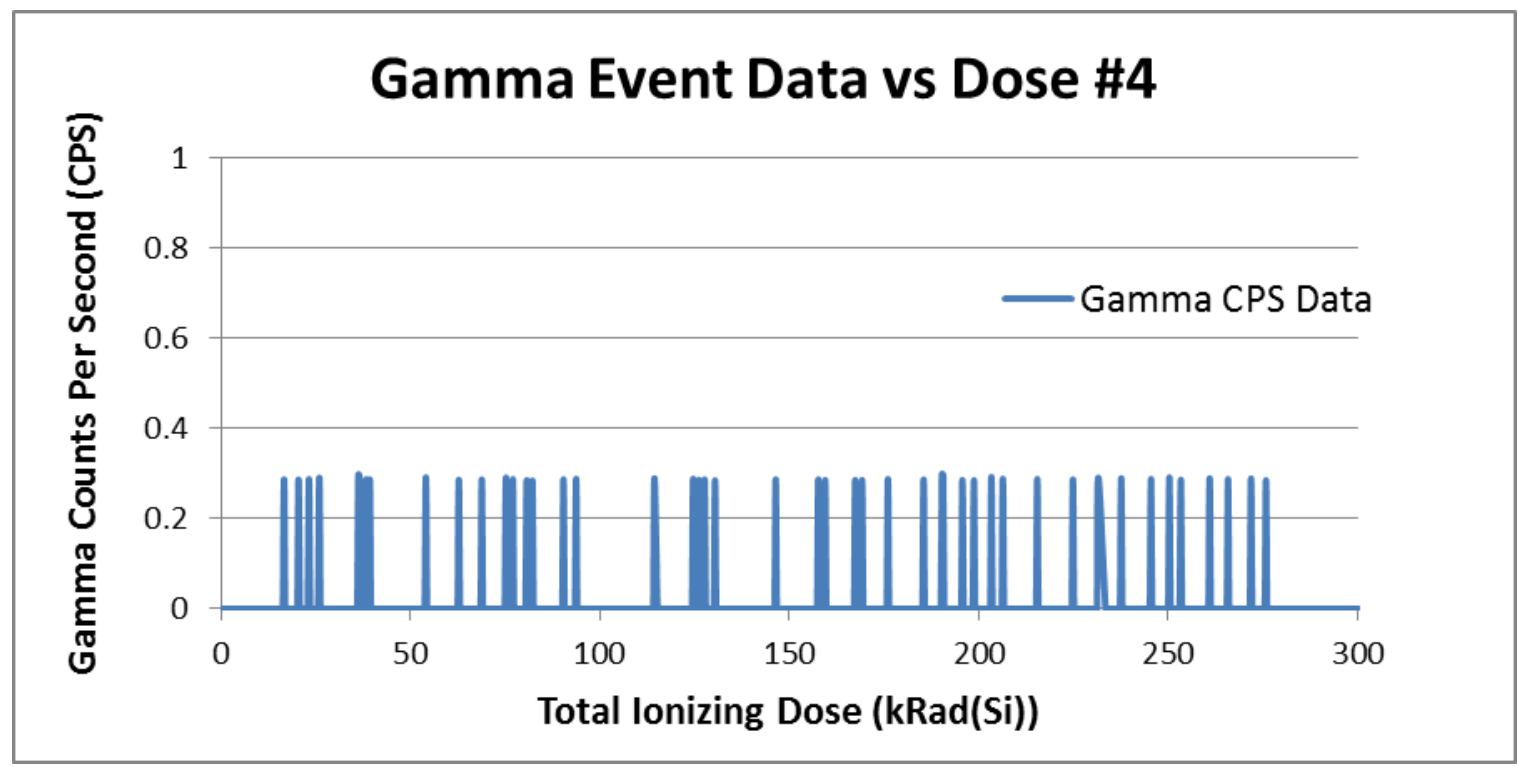

Fig. 51. System 4 gamma count rate vs TID radiation. 


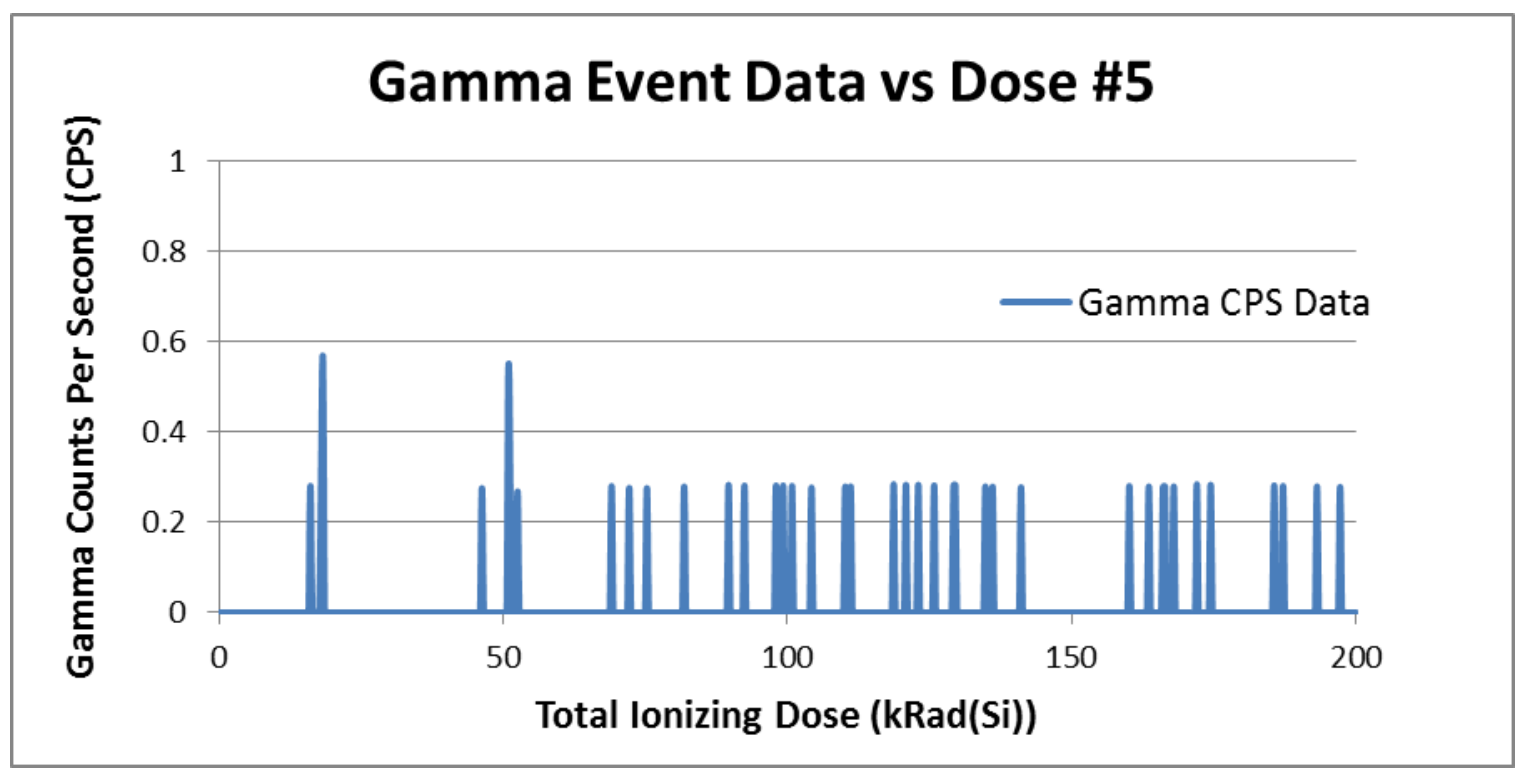

Fig. 52. System 5 gamma count rate vs TID radiation.

\section{CONCLUSION}

We have presented the results of the NEET 2 project "Radiation-Hardened Circuitry Using MaskProgrammable Analog Arrays" [1]. This project included complete system design and pre-irradiation calibration across pertinent temperature and pressure levels as well as performance validation as a function of TID radiation. LabVIEW software was also updated to allow for simplistic display of continuous time data that were converted from raw digital form using as-found calibration coefficients. We performed testing and evaluation of the systems pre- and post-radiation and found the systems to be robust and appropriate for rad-hard robotics applications.

\section{REFERENCES}

1. C. L. Britton, M. N. Ericson, and B. Blalock, "Radiation-Hardened Circuitry Using MaskProgrammable Analog Arrays," proposal submitted under NEET-2: Advanced Sensors and Instrumentation, 2012.

2. G. F. Knoll, Radiation Detection and Measurement, $4^{\text {th }}$ ed., 2010, p. 131 .

3. Reconfigurable Coarse-Gain Analog Arrays, Phase 2 Final Report (Restricted distribution).

4. M. N. Ericson, High-Temperature, High-Resolution A/D Conversion Using $2^{\text {nd }}$ - and $4^{\text {th }}$-Order Cascaded $\Sigma \Delta$ Modulation in 3.3-V 0.5um SOS-CMOS, Ph.D. Dissertation, The University of Tennessee, Knoxville, August 2002.

Eric Bainville, http://www.bealto.com/home.html. 
